REPRESENTATION THEORY

An Electronic Journal of the American Mathematical Society

Volume 14, Pages 201-248 (February 15, 2010)

S 1088-4165(10)00367-5

\title{
UNITARY GENUINE PRINCIPAL SERIES OF THE METAPLECTIC GROUP
}

\author{
ALESSANDRA PANTANO, ANNEGRET PAUL, AND SUSANA A. SALAMANCA-RIBA
}

\begin{abstract}
This paper presents some recent progress on the classification of the unitary genuine irreducible representations of the metaplectic group $M p(2 n)$. Our focus will be on Langlands quotients of genuine minimal principal series; the main result is an embedding of the set of unitary parameters of such representations into the union of spherical unitary parameters for certain split orthogonal groups. The latter are known from work of D. Barbasch; hence we obtain the non-unitarity of a large (conjecturally complete) set of parameters for Langlands quotients of genuine principal series of $M p(2 n)$.
\end{abstract}

\section{INTRODUCTION}

The goal of this paper is to present some recent progress on the classification of the unitary genuine irreducible representations of the metaplectic group. The focus is on Langlands quotients of genuine minimal principal series of $M p(2 n)$; our main result is an embedding of the set of unitary parameters of such representations into the union of spherical unitary parameters for certain split orthogonal groups. The latter are known from [4; hence we obtain the non-unitarity of a large (conjecturally complete) set of parameters for Langlands quotients of genuine principal series of $M p(2 n)$. For the pseudospherical case, this result already appears in [3. The authors of 3 prove that a similar embedding holds for all non-trivial coverings of simple split groups, but they restrict their attention to the pseudospherical case. In this paper, the emphasis is exclusively on $M p(2 n)$, but the pseudospherical restriction is removed. In the specific example of the pseudospherical principal series of $M p(2 n)$, the embedding in 3 turns out to be a bijection. We conjecture that this result holds for all genuine principal series of $M p(2 n)$ and provide some evidence for this claim.

Let $G=M p(2 n)$ be the (unique) non-trivial two-fold cover of the symplectic group $S p(2 n, \mathbb{R})$. Choose a minimal parabolic subgroup of $S p(2 n, \mathbb{R})$, and let $M A$ be the inverse image of its Levi factor in $M p(2 n)$. Then $M$ is a finite abelian group of order $2^{n+1}$, and $A$ is a vector group. For every irreducible representation $\delta$ of $M$ and every character $\nu$ of $A$, we choose a nilpotent group $N$ such that $M A N$ is the inverse image of a minimal parabolic subgroup of $S p(2 n, \mathbb{R})$, and $\nu$ is weakly dominant for the positive root system determined by $N$. The representation $\delta \otimes \nu$ of $M A$ can be regarded as a representation of $M A N$, with $N$ acting trivially. The

Received by the editors May 26, 2009.

2010 Mathematics Subject Classification. Primary 22E45.

This research was supported by NSF grants DMS 0554278 and DMS 0201944.

(C)2010 American Mathematical Society Reverts to public domain 28 years from publication 
corresponding induced representation is a principal series representation of $M p(2 n)$, denoted by

$$
I(\delta, \nu):=\operatorname{Ind}_{M A N}^{M p(2 n)}(\delta \otimes \nu) .
$$

Note that $I(\delta, \nu)$ is independent of the choice of $N$ and is unitary if the character $\nu$ of $A$ is unitary (because we use normalized induction). We restrict our attention to representations of $M p(2 n)$ that are genuine, in the sense that they do not factor to $S p(2 n, \mathbb{R})$. It is easy to check that $I(\delta, \nu)$ is genuine if and only if the $M$-type $\delta$ does not factor to the appropriate subgroup of $S p(2 n, \mathbb{R})$; i.e., if $\delta$ is a genuine $M$-type. Every genuine representation $\delta$ of $M$ is contained in a unique fine $K$-type $\mu_{\delta}$ (see Definition 3.6). The distinguished irreducible composition factor of $I(\delta, \nu)$ containing $\mu_{\delta}$ is a Langlands quotient of $M p(2 n)$, denoted by $J(\delta, \nu)$.

We are interested in the set of parameters $\nu$ for which the irreducible representation $J(\delta, \nu)$ is unitary. Note that $\nu$ is real if and only if $J(\delta, \nu)$ has real infinitesimal character. By a result of Vogan (see 11, Theorem 16.10), any unitary representation with non-real infinitesimal character is unitarily induced from a unitary representation with real infinitesimal character on a proper Levi subgroup. Hence we will (as we may) assume that $\nu$ is real. We call the set

$$
C S(M p(2 n), \delta):=\left\{\nu \in \mathfrak{a}_{\mathbb{R}}^{*}: J(\delta, \nu) \text { is unitary }\right\}
$$

the $\delta$-complementary series of $M p(2 n)$. By work of Knapp and Zuckerman, every genuine Langlands quotient $J(\delta, \nu)$ admits a Hermitian form which is invariant under the action of the group; the $\delta$-complementary series of $M p(2 n)$ consists of all the parameters $\nu$ which make this form (positive) definite. To better describe the results we need to introduce some notation. The Weyl group $W$ of $M p(2 n)$ acts on the set $\widehat{M}$ of irreducible representations of $M$, and on the set $\mathfrak{a}_{\mathbb{R}}^{*}$ of real characters of $A$. Because

$$
\omega \cdot C S(M p(2 n), \delta)=C S(M p(2 n), \omega \cdot \delta) \quad \forall \omega \in W,
$$

the $\delta$-complementary series of $M p(2 n)$ is invariant under the action of the stabilizer $W^{\delta}$ of $\delta$ in $W$. Equation (1.3) also shows that it is sufficient to compute the complementary series associated to a single $M$-type in each orbit of $\widehat{M}$ under the action of the Weyl group. $W$-orbits of genuine $M$-types of $M p(2 n)$ are parameterized by pairs of non-negative integers $(p, q)$ with $p+q=n$; the selected representative in the $(p, q)$-orbit will be denoted by $\delta_{p, q}$. We prove that the $\delta_{p, q}$-complementary series of $M p(2 n)$ embeds into the product of the spherical complementary series $C S\left(S O(p+1, p)_{o}, \delta_{0}\right)$ of $S O(p+1, p)_{o}$ with the spherical complementary series of $S O(q+1, q)_{o}$. Here is a more precise statement of the result:

Theorem 1.1. Let $G=M p(2 n)$ and let $\nu=\left(\nu_{1}, \ldots, \nu_{n}\right)$ be a real character of $A$. For each pair of non-negative integers $(p, q)$ such that $p+q=n$, write $\nu=\left(\nu^{p} \mid \nu^{q}\right)$ with

$$
\nu^{p}:=\left(\nu_{1}, \ldots, \nu_{p}\right) \text { and } \nu^{q}:=\left(\nu_{p+1}, \ldots, \nu_{n}\right) .
$$

If the spherical Langlands quotient $J\left(\delta_{0}, \nu^{p}\right)$ of $S O(p+1, p)_{0}$ and/or the spherical Langlands quotient $J\left(\delta_{0}, \nu^{q}\right)$ of $S O(q+1, q)_{0}$ are not unitary, then the genuine Langlands quotient $J\left(\delta_{p, q}, \nu\right)$ of $M p(2 n)$ is also not unitary.

Because the spherical unitary dual of real split orthogonal groups is known (by work of Barbasch), we obtain explicit non-unitarity certificates for genuine principal series for $M p(2 n)$. Here is an equivalent formulation of Theorem 1.1 
Theorem 1.2. Let $G=M p(2 n)$ and let $\nu=\left(\nu_{1}, \ldots, \nu_{n}\right)$ be a real character of $A$. Write $\nu=\left(\nu^{p} \mid \nu^{q}\right)$, as in (1.4). There is a well-defined injection

$$
C S\left(M p(2 n), \delta_{p, q}\right) \rightarrow C S\left(S O(p+1, p)_{0}, \delta_{0}\right) \times C S\left(S O(q+1, q)_{0}, \delta_{0}\right)
$$

taking

$$
\nu \mapsto\left(\nu^{p}, \nu^{q}\right)
$$

Conjecture 1.3. The map (1.5) is a bijection.

As an example, we include the picture of the spherical unitary duals of $S O(2,1)_{0}$ and $S O(3,2)_{0}$, and of the $\delta_{2,1}$-complementary series of $M p(6)$. See Figure 1 .

We briefly discuss the relation between Conjecture 1.3 and the "omega-regular conjecture" introduced in [14. In [14, we defined the notion of an omega-regular representation for the metaplectic group. Roughly, a representation of $M p(2 n)$ is omega-regular if its infinitesimal character is at least as regular as that of the oscillator representation. Generalizing the idea of an admissible $A_{\mathfrak{q}}(\lambda)$-module of [17, we displayed a family of unitary omega-regular representations called $A_{\mathfrak{q}}(\Omega)$ modules, and conjectured that these modules exhaust the genuine omega-regular unitary dual of $M p(2 n)$. If $\pi$ is an irreducible principal series representation of the metaplectic group, then $\pi$ is an $A_{\mathfrak{q}}(\Omega)$-module if and only if it is an even oscillator representation. Then, for principal series representations, the "omega-regular conjecture" states that a genuine omega-regular irreducible principal series representation of $M p(2 n)$ is unitary if and only if it is an even oscillator representation. This result follows from Theorem 1.2. Hence, we obtain the following corollary.

Corollary 1.4. The "omega-regular conjecture" is true for all principal series representations of $M p(2 n)$.

In addition, for genuine non-unitary omega-regular principal series representations, we obtain non-unitarity certificates on specific petite $\widetilde{U}(n)$-types, which provide an important ingredient for proving the general conjecture. A complete proof of the "omega-regular conjecture" will appear in a separate paper [15].

The technique used in the proof of Theorem 1.1 follows Barbasch's idea to use calculations on petite $K$-types to compare unitary parameters for different groups. For each genuine $M$-type $\delta$, let $\Delta_{\delta}$ be the system of good roots for $\delta$, as in Section 3.4. and let $G^{\delta}$ be the connected real split group whose root system is dual to $\Delta_{\delta}$. If $\delta=\delta_{p, q}$, then

$$
\Delta_{\delta} \text { is a root system of type } C_{p} \times C_{q} \text {, }
$$

and $G^{\delta}=S O(p+1, p)_{0} \times S O(q+1, q)_{0}$. Theorem 1.2 asserts that the set of unitary parameters for a (Hermitian) genuine Langlands quotient $J(\delta, \nu)$ of $M p(2 n)$ embeds into the set of unitary parameters for a (Hermitian) spherical Langlands quotient of $G^{\delta}$. Associated to $\delta$, there is a family of Hermitian intertwining operators $T\left(w_{0}, \mu, \delta, \nu\right)$, one for each $K$-type $\mu$, with the property that the Langlands quotient $J(\delta, \nu)$ is unitary if and only if

$$
T\left(w_{0}, \mu, \delta, \nu\right) \text { is positive semi-definite, for all } \mu \in \widehat{K} \text {. }
$$

The domain of the operator $T\left(w_{0}, \mu, \delta, \nu\right)$, i.e., the space $V_{\mu}[\delta]:=\operatorname{Hom}_{M}(\mu, \delta)$, carries a representation of the stabilizer $W^{\delta}$ of $\delta$ in $W$. If the $K$-type $\mu$ is petite (see Definition (3.6)), the operator $T\left(w_{0}, \mu, \delta, \nu\right)$ depends only on the $W^{\delta}$-structure of $V_{\mu}[\delta]$ and behaves like a spherical operator for the group $G^{\delta}$. Note that for all 


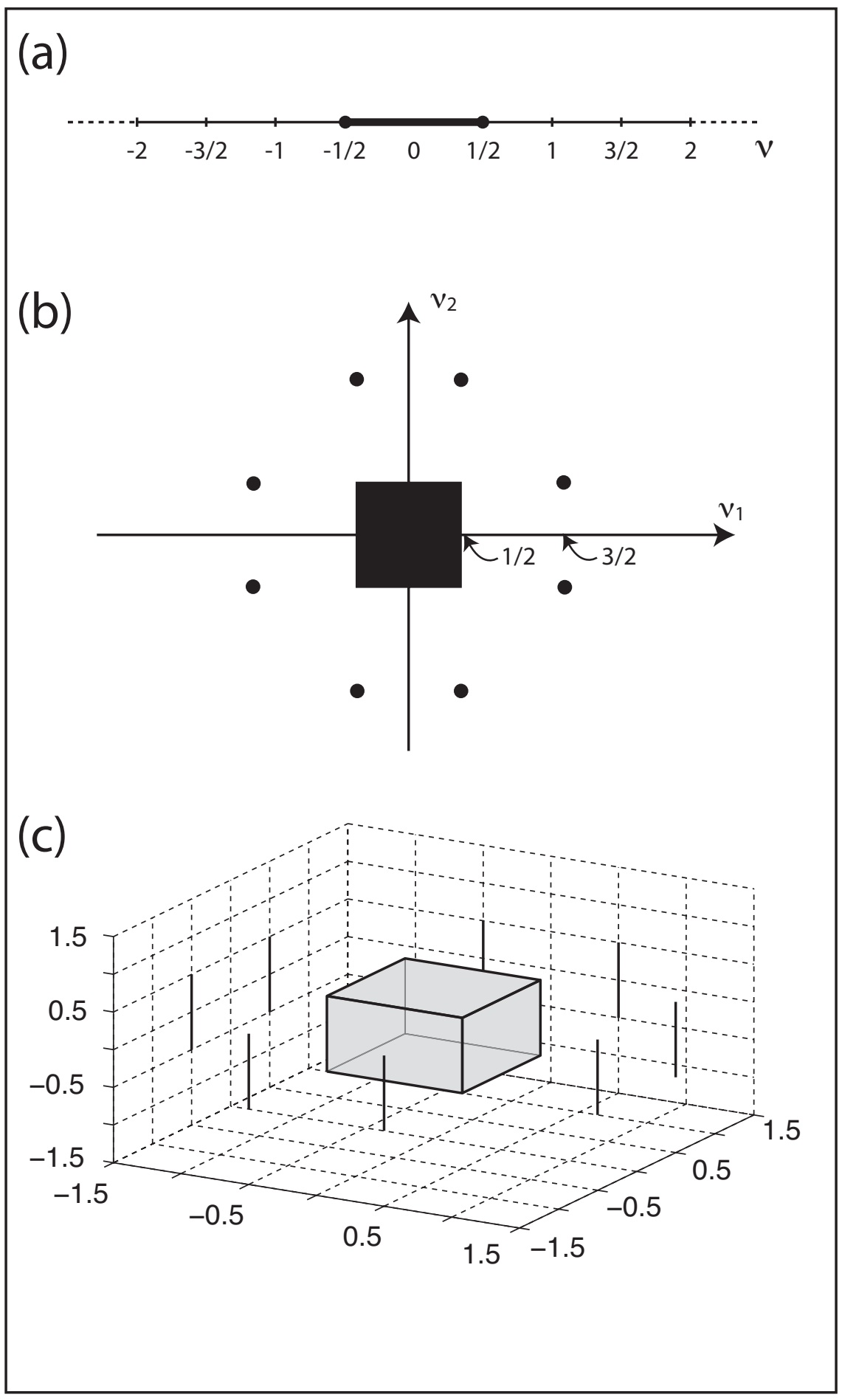

Figure 1 . The sets $C S\left(S O(2,1)_{0}, \delta_{0}\right), C S\left(S O(3,2)_{0}, \delta_{0}\right)$ and $C S\left(M p(6), \delta_{2,1}\right)$. 
genuine $\delta, W^{\delta}$ coincides with the Weyl group of the system of good roots, hence with the Weyl group of $G^{\delta}$. Therefore, this matching of intertwining operators ties the unitarity of a genuine Langlands quotient $J(\delta, \nu)$ of $M p(2 n)$ to the unitarity of a spherical representation of $G^{\delta}$.

Proving Conjecture 1.3 amounts to showing that for each pair of parameters

$$
\nu^{p} \in C S\left(S O(p+1, p)_{0}, \delta_{0}\right) \quad \text { and } \quad \nu^{q} \in C S\left(S O(q+1, q)_{0}, \delta_{0}\right),
$$

the Langlands quotient $J\left(\delta_{p, q},\left(\nu^{p} \mid \nu^{q}\right)\right)$ of $M p(2(p+q))$ is unitary. We do this completely for $p+q \leq 3$. For the general case, we produce two large families of spherical unitary parameters for $S O(p+1, p)_{0} \times S O(q+1, q)_{0}$ which give rise to $\delta_{p, q}$-complementary series of $M p(2(p+q))$. One can construct many more examples; we plan to pursue this in a future paper.

Some comments are in order. Barbasch's method of computing some intertwining operators purely in terms of Weyl group representations is central to this paper. The same technique has proven successful in the past: it was used in [5] to prove that the spherical unitary dual of a real split group embeds into the spherical unitary dual of the corresponding $p$-adic split group, and in 3] to prove that the pseudospherical unitary dual of non-trivial coverings of split simple groups embeds into the spherical unitary dual of certain linear groups. Generalizing these ideas to genuine non-pseudospherical representations is non-trivial, because the intertwining operators are harder to compute, due to the presence of bad roots.

We give an outline of the paper. Since understanding the techniques used in the determination of the spherical unitary dual of split real classical groups is crucial for our argument, we summarize the main results of [5] in Section 2. Section 3 is devoted to the structure of the group $M p(2 n)$. The genuine complementary series of $M p(2 n)$ are introduced in Section 4 In Section 5, we discuss the structure of the space $\operatorname{Hom}_{M}(\mu, \delta)$ and the corresponding $W^{\delta}$-representation. Next, in Section 6 , we review the theory of intertwining operators for minimal principal series of $M p(2 n)$ and we prove some preliminary results. In Section 7 , we explain the role of petite $K$-types for producing non-unitarity certificates, and we state the main theorem. The actual proof of the main theorem is contained in Section 10. In Section 8, we present some evidence for Conjecture 1.3. The irreducible representations of Weyl groups of type $C$ are described in Section 9 Finally, in the appendix, we give an explicit description of the spherical unitary dual of split groups of type $B$ as in [4.

Sections 5 6 and 7 are based on unpublished work of D. Barbasch and the first author on non-unitarity certificates for non-spherical principal series (for more general cases than $M p(2 n)$ ), cf. [ $[$. We thank D. Barbasch for generously sharing his ideas. In addition, we would like to thank D. Vogan, P. Trapa and D. Ciubotaru for offering valuable suggestions along the way, and the authors of [3] for providing such an interesting inspiration.

\section{SPHERICAL UNITARY DUAL OF REAL CLASSICAL SPLIT GROUPS}

The purpose of this section is to summarize some of the results we need about the spherical unitary dual of real split groups. For a more detailed description, the interested reader can consult [6].

Let $G$ be the set of real points of a connected linear reductive group defined over $\mathbb{R}$. Let $K$ be a maximal compact subgroup of $G$, let $M A$ be the Levi factor of a minimal parabolic subgroup of $G$ (with compact part $M$ ) and let $W$ be the Weyl 
group of $G$. Assume that $G$ is a split group of rank $n$, so that $M A$ is isomorphic to $\left(\mathbb{R}^{\times}\right)^{n}$ and $M$ is isomorphic to $(\mathbb{Z} / 2 \mathbb{Z})^{n}$.

Theorem 2.1 (cf. [21]). Let $\delta_{0}$ be the trivial $M$-type, and let $\nu$ be a character of A. Choose a minimal parabolic subgroup $P=M A N$ of $G$ making $\nu$ dominant, and let

$$
I\left(\delta_{0}, \nu\right):=\operatorname{Ind}_{M A N}^{G}\left(\delta_{0} \otimes \nu \otimes 1\right)
$$

be the spherical principal series representation of $G$ induced from $\nu$. Then:

- $I\left(\delta_{0}, \nu\right)$ has a unique irreducible spherical subquotient, denoted by $J\left(\delta_{0}, \nu\right)$.

- $J\left(\delta_{0}, \nu\right)$ is equivalent to $J\left(\delta_{0}, \nu^{\prime}\right)$ if and only if $\nu=w \nu^{\prime}$, for some $w \in W$.

- $J\left(\delta_{0}, \nu\right)$ is Hermitian if and only if $w \nu=\bar{\nu}^{-1}$, for some $w \in W$.

Remark 2.2. Because $A$ is a vector group, we can interpret the character $\nu$ of $A$ as a complex linear functional on the Lie algebra of $A$. For the purpose of unitarity, we can also assume that $\nu$ is real (see [11, Theorem 16.10, or 7]). The Hermitian condition becomes: $w \nu=-\nu$, for some $w \in W$. (The element $w$ can be assumed to be the long Weyl group element $w_{0}$.)

Suppose that $\nu \in \mathfrak{a}_{\mathbb{R}}^{*}$ is dominant and satisfies the condition $w_{0} \cdot \nu=-\nu$, with $w_{0}$ the long element in the Weyl group. Then the Langlands quotient $J\left(\delta_{0}, \nu\right)$ is an irreducible Hermitian spherical representation of $G$. The unitarity of $J\left(\delta_{0}, \nu\right)$ depends on the signature of certain intertwining operators. Precisely, there is one operator

$$
T\left(w_{0}, \mu, \delta_{0}, \nu\right): \operatorname{Hom}_{K}\left(E_{\mu}, I\left(\delta_{0}, \nu\right)\right) \rightarrow \operatorname{Hom}_{K}\left(E_{\mu}, I\left(\delta_{0},-\nu\right)\right)
$$

for every irreducible quasi-spherical representation $\left(\mu, E_{\mu}\right)$ of $K$. (A $K$-type $\mu$ is called quasi-spherical if its restriction to $M$ contains the trivial $M$-type.) The spherical Langlands quotient $J\left(\delta_{0}, \nu\right)$ is unitary if and only if each operator $T\left(w_{0}, \mu, \delta_{0}, \nu\right)$ is positive semi-definite.

By Frobenius reciprocity, we can interpret $T\left(w_{0}, \mu, \delta_{0}, \nu\right)$ as an operator on the space $V_{\mu}\left[\delta_{0}\right]:=\operatorname{Hom}_{M}\left(\mu, \delta_{0}\right) \simeq\left(E_{\mu}^{*}\right)^{M}$. When $\mu$ is the trivial $K$-type, i.e., the fine $K$-type $\mu_{\delta_{0}}$, the space $V_{\mu}\left[\delta_{0}\right]$ is one-dimensional; hence the operator $T\left(w_{0}, \mu, \delta_{0}, \nu\right)$ acts on it by a scalar. We normalize the operators so that this scalar is 1 . Let

$$
w_{0}=s_{\alpha_{r}} s_{\alpha_{r-1}} \cdots s_{\alpha_{1}}
$$

be a minimal decomposition of $w_{0}$ as a product of simple reflections. The operator $T\left(w_{0}, \mu, \delta_{0}, \nu\right)$ inherits a similar factorization:

$$
T\left(w_{0}, \mu, \delta_{0}, \nu\right)=\prod_{j=1}^{r} T\left(s_{\alpha_{j}}, \mu, \delta_{0}, \nu_{j-1}\right)
$$

with $\nu_{0}=\nu$ and $\nu_{j}=\underbrace{s_{\alpha_{j}} s_{\alpha_{j-1}} \cdots s_{\alpha_{1}}}_{x_{j}} \cdot \nu=x_{j} \cdot \nu$ for all $j>1$. Every factor

$$
T\left(s_{\alpha}, \mu, \delta_{0}, \gamma\right): V_{\mu}\left[\delta_{0}\right] \rightarrow V_{\mu}\left[\delta_{0}\right]
$$

is an endomorphism of the space $V_{\mu}\left[\delta_{0}\right]$. To construct $T\left(s_{\alpha}, \mu, \delta_{0}, \gamma\right)$, we look at the $S L(2, \mathbb{R})$-subgroup $G_{\alpha}$ associated to $\alpha$, and we pick a generator $Z_{\alpha}$ for the Lie algebra of $G_{\alpha} \cap K \simeq S O(2)$. Then, we decompose $E_{\mu}$ as a direct sum of generalized eigenspaces for $\mathrm{d} \mu\left(Z_{\alpha}\right)$ and let

$$
V_{\mu}\left[\delta_{0}\right]=\bigoplus_{k \in \mathbb{N}} \operatorname{Hom}_{M}\left(V_{\mu}( \pm 2 k), \delta_{0}\right)
$$


be the corresponding decomposition of $V_{\mu}\left[\delta_{0}\right]$. Here we use $\mathbb{N}$ to denote the set of non-negative integers, and we set $V_{\mu}(s):=\left\{v \in E_{\mu} \mid \mathrm{d} \mu\left(i Z_{\alpha}\right) v=s v\right\}$, for all $s \in \mathbb{Z}$. The $\alpha$-factor $T\left(s_{\alpha}, \mu, \delta_{0}, \gamma\right)$ acts on the space $\operatorname{Hom}_{M}\left(V_{\mu}( \pm 2 k), \delta_{0}\right)$ by the scalar:

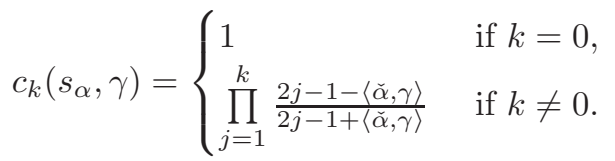

Remark 2.3. Let $\mu$ be a quasi-spherical $K$-type. The eigenvalues of the operator $T\left(w_{0}, \mu, \delta_{0}, \nu\right)$ can vanish only on the reducibility hyperplanes:

$$
\langle\check{\beta}, \nu\rangle=2 n+1, \quad \beta \in \Delta^{+}, n \in \mathbb{Z} .
$$

In the open regions in the complement of this hyperplane arrangement, the principal series $I\left(\delta_{0}, \nu\right)$ is irreducible (hence equal to $\left.J\left(\delta_{0}, \nu\right)\right)$ and the operators $T\left(w_{0}, \mu, \delta_{0}\right.$, $\nu)$ have constant signature. In particular, if $\nu$ belongs to the fundamental alcove

$$
\mathcal{C}_{0}=\left\{\nu \in \mathfrak{a}_{\mathbb{R}}^{*}: 0 \leq\langle\breve{\beta}, \nu\rangle<1, \forall \beta \in \Delta^{+}\right\},
$$

then all the operators $T\left(w_{0}, \mu, \delta_{0}, \nu\right)$ are positive definite and $J\left(\delta_{0}, \nu\right)$ is unitary.

Figure 2 shows a picture of the spherical unitary dual of $S O(3,2)_{0}$, along with the reducibility hyperplanes.

2.0.1. Quasi-spherical petite $K$-types. Let $\mu$ be a quasi-spherical $K$-type. The Weyl group $W$ acts on the space

$$
V_{\mu}\left[\delta_{0}\right]:=\operatorname{Hom}_{M}\left(\mu, \delta_{0}\right) \simeq\left(E_{\mu}^{*}\right)^{M}
$$

by:

$$
w \cdot T(v)=T\left(\mu\left(\sigma^{-1} v\right)\right) \quad \forall v \in E_{\mu}, T \in V_{\mu}\left[\delta_{0}\right], w \in W .
$$

(Here $\sigma$ denotes a representative for $w$ in the normalizer of $A$ in $K$.)

Definition 2.4. A quasi-spherical $K$-type is called petite if its level is less than or equal to 2 .

(The definition of level is recalled in Section 3.6) If $\mu$ is petite, the intertwining operator $T\left(w_{0}, \mu, \delta, \nu\right)$ can be computed by means of Weyl group calculations, and depends only on the $W$-representation on $V_{\mu}\left[\delta_{0}\right]$. To make this precise, we need to introduce some notation.

Notation. Let

$$
w_{0}=s_{\beta_{r}} \cdots s_{\beta_{2}} s_{\beta_{1}}
$$

be a minimal decomposition of $w_{0}$ into simple reflections in $W$. For each $W$-type $\psi$, and each (real) character $\nu$ of $A$, set:

$$
A\left(w_{0}, \psi, \nu\right):=\prod_{j=1}^{r} A\left(s_{\beta_{j}}, \psi, \nu_{j-1}\right)
$$

with $\nu_{0}=\nu, \nu_{j}=s_{\beta_{j}} s_{\beta_{j-1}} \cdots s_{\beta_{1}} \cdot \nu$ for $j>1$, and

$$
A\left(s_{\beta_{j}}, \psi, \nu_{j-1}\right):=\frac{I d+\left\langle\check{\beta}_{j}, \nu_{j-1}\right\rangle \psi\left(s_{\beta_{j}}\right)}{1+\left\langle\check{\beta}_{j}, \nu_{j-1}\right\rangle} .
$$




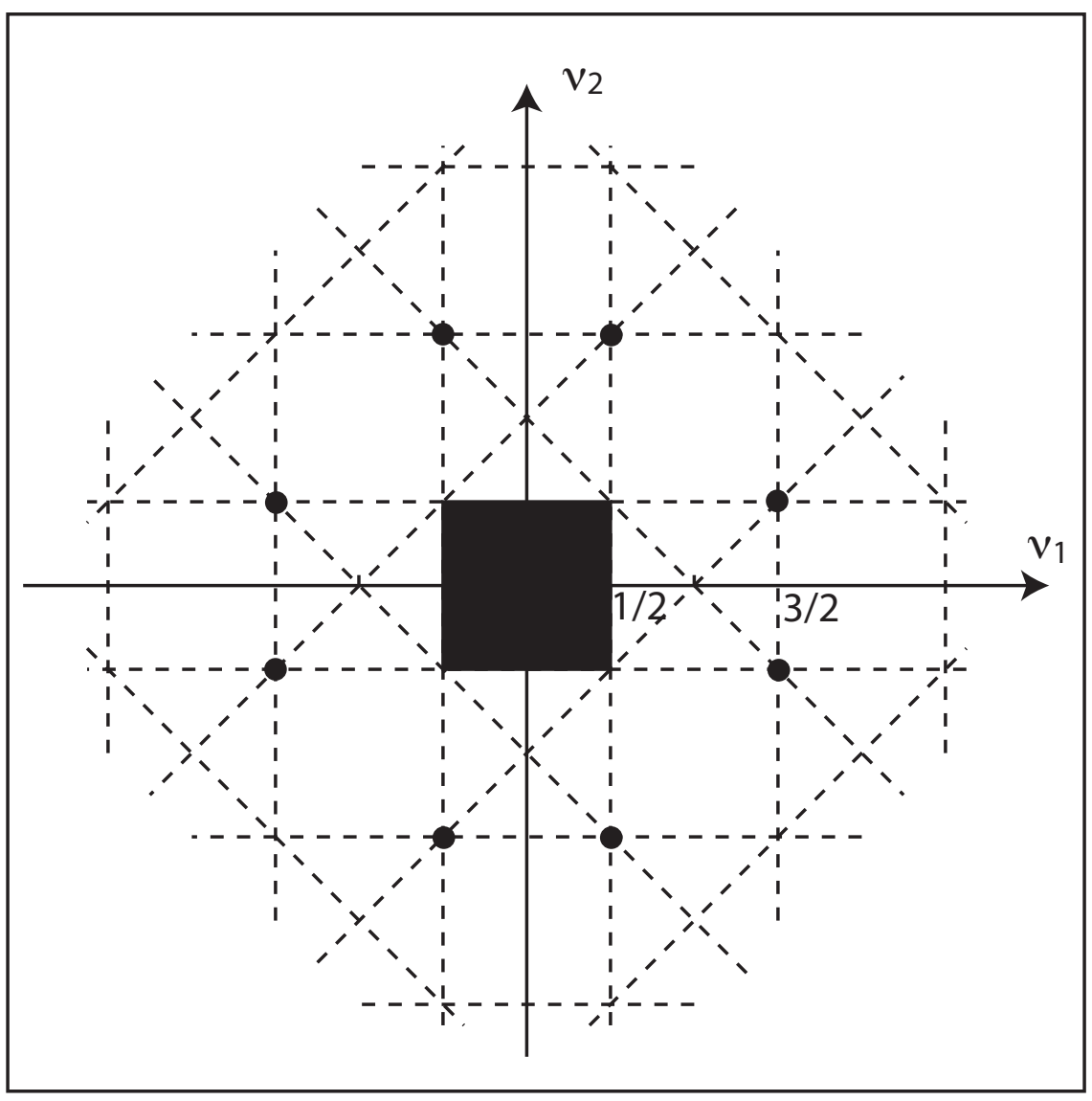

Figure 2. The spherical unitary dual of $S O(3,2)_{0}$.

Theorem 2.5 (cf. [5). Let $\mu$ be a quasi-spherical $K$-type, and let $\psi_{\mu}$ be the representation of $W$ on the space

$$
V_{\mu}\left[\delta_{0}\right]:=\operatorname{Hom}_{M}\left(\mu, \delta_{0}\right) \simeq\left(E_{\mu}^{*}\right)^{M}
$$

defined in equation (2.8). If $\mu$ is petite, the intertwining operator $T\left(w_{0}, \mu, \delta_{0}, \nu\right)$ depends only on the $W$-structure of $V_{\mu}\left[\delta_{0}\right]$. Precisely:

$$
T\left(w_{0}, \mu, \delta_{0}, \nu\right)=A\left(w_{0}, \psi_{\mu}, \nu\right),
$$

for all $\nu \in \mathfrak{a}_{\mathbb{R}}^{*}$.

Remark 2.6. Let $G(\mathbb{F})$ be the split $p$-adic group corresponding to the root system of $G$. For each $\nu \in \mathfrak{a}_{\mathbb{R}}^{*}$, let $I_{G(\mathbb{F})}(\nu)$ be the principal series of $G(\mathbb{F})$ induced from $\nu$ and let $J_{G(\mathbb{F})}(\nu)$ be its unique irreducible subquotient. Assume that $w_{0} \cdot \nu=-\nu$, so that $J_{G(\mathbb{F})}(\nu)$ is Hermitian. The operators

$$
\left\{A\left(w_{0}, \psi, \nu\right): \psi \in \widehat{W}\right\}
$$


are exactly the intertwining operators needed to determine the unitarity of $J_{G(\mathbb{F})}(\nu)$ : the spherical Langlands quotient $J_{G(\mathbb{F})}(\nu)$ is unitary if and only if

$$
A\left(w_{0}, \psi, \nu\right) \text { is positive semi-definite } \forall \psi \in \widehat{W} \text {. }
$$

(To be precise, we should mention that the operators $A\left(w_{0}, \psi, \nu\right)$ are actually operators for the Hecke algebra associated to $G(\mathbb{F})$. See [6] for details.)

2.0.2. Relevant $W$-types. The set of relevant $W$-types is a minimal set of $W$-types which is sufficient to detect unitarity for $p$-adic spherical Langlands quotients.

Definition 2.7 (cf. [3]). A set $\Sigma \subset \widehat{W}$ detects spherical unitarity if each Hermitian spherical Langlands quotient $J_{G(\mathbb{F})}(\nu)$ is unitary if and only if

$$
A\left(w_{0}, \psi, \nu\right) \text { is positive semi-definite } \forall \psi \in \Sigma \text {. }
$$

Theorem 2.8 (cf. [4, [5]). For split groups $G$ of type $B_{n}$ or $C_{n}$, the following sets of $W$-types are relevant:

$$
\{(n-m, m) \times(0): 0 \leq m \leq[n / 2]\} \cup\{(n-m) \times(m): 0 \leq m \leq n\} .
$$

The labeling of $\widehat{W}$ is the standard labeling by partitions and pairs of partitions (see Section 9).

Theorem 2.9 (cf. [5]). Let $G$ be a real split group, with Weyl group $W$. For every relevant $W$-type $\psi$, there exists a quasi-spherical petite $K$-type $\mu$ such that

$$
T\left(w_{0}, \mu, \delta_{0}, \nu\right)=A\left(w_{0}, \psi, \nu\right)
$$

for all $\nu \in \mathfrak{a}_{\mathbb{R}}^{*}$.

This matching of operators allows us to compare spherical unitary parameters for $G$ and $G(\mathbb{F})$.

2.0.3. An embedding of spherical unitary duals. Suppose that $J\left(\delta_{0}, \nu\right)$ is a Hermitian spherical Langlands quotient for a real split group $G$. If $J\left(\delta_{0}, \nu\right)$ is unitary, then the operator $T\left(w_{0}, \mu, \delta_{0}, \nu\right)$ is positive semi-definite for every quasi-spherical $K$-type $\mu$. In particular,

$$
T\left(w_{0}, \mu, \delta_{0}, \nu\right) \text { is positive semi-definite for all } \mu \in \widehat{K} \text { petite. }
$$

Theorem 2.9 implies that

$$
A\left(w_{0}, \psi, \nu\right) \text { is positive semi-definite for all } \psi \in \widehat{W} \text { relevant. }
$$

Because relevant $W$-types detect spherical unitarity for $p$-adic split groups, we deduce that the Hermitian spherical Langlands quotient $J_{\mathbb{F}}(\nu)$ (of $G(\mathbb{F})$ ) is unitary. Hence the set of spherical unitary parameters for the real group $G$ embeds in the corresponding set for the $p$-adic group $G(\mathbb{F})$. (Barbasch has proved that this embedding of spherical unitary duals is actually an equality for classical split groups.)

Theorem 2.10 (cf. 5). The spherical unitary dual of a classical split group is independent of the field.

Corollary 2.11. Let $G$ be a real split classical group, with Weyl group $W$ and long Weyl group element $w_{0}$. A Hermitian spherical Langlands quotient $J\left(\delta_{0}, \nu\right)$ of $G$ is unitary if and only if

$$
A\left(w_{0}, \psi, \nu\right) \text { is positive semi-definite for all } \psi \in \widehat{W} \text { relevant. }
$$


Corollary 2.12. Quasi-spherical petite K-types detect unitarity for spherical Langlands quotients of real split classical groups.

\section{The structure of $M p(2 n)$}

Let $G=M p(2 n)$ be the connected double cover of the real split group

$$
S p(2 n, \mathbb{R})=\left\{g \in G L(2 n, \mathbb{R}): g^{t}\left(\begin{array}{cc}
0 & I_{n} \\
-I_{n} & 0
\end{array}\right) g=\left(\begin{array}{cc}
0 & I_{n} \\
-I_{n} & 0
\end{array}\right)\right\},
$$

with $I_{n}$ the $n \times n$ identity matrix. We denote by $\mathfrak{g}_{0}$ the Lie algebra of $G$ :

$$
\mathfrak{g}_{0}=\mathfrak{s p}(2 n, \mathbb{R})=\left\{\left(\begin{array}{cc}
A & B \\
C & -A^{T}
\end{array}\right): B \text { and } C \text { are symmetric }\right\},
$$

and by $\mathfrak{g}$ its complexification. Let $\mathfrak{k}_{0}$ be the maximal compact Cartan subalgebra of $\mathfrak{g}_{0}$ corresponding to the Cartan involution $\theta(X)=-X^{t}$ :

$$
\mathfrak{k}_{0}=\left\{\left(\begin{array}{cc}
A & B \\
-B & A
\end{array}\right): A \text { is skew-symmetric, and } B \text { is symmetric }\right\},
$$

and let $K$ be the corresponding maximal compact subgroup of $G$. Notice that $\mathfrak{k}_{0}$ is isomorphic to $\mathfrak{u}(n)$ via

$$
\left(\begin{array}{cc}
A & B \\
-B & A
\end{array}\right) \mapsto A+i B
$$

and that $K$ is isomorphic to $\widetilde{U}(n)$ (the connected double cover of $U(n)$ ). We identify $K$ with a subgroup of $U(n) \times U(1)$ :

$$
K=\left\{[g, z] \in U(n) \times U(1): \operatorname{det}(g)=z^{2}\right\},
$$

and $\mathfrak{k}_{0}$ with a subalgebra of $\mathfrak{u}(n) \oplus \mathfrak{u}(1)$ :

$$
\mathfrak{k}_{0}=\{[X, t] \in \mathfrak{u}(n) \oplus \mathfrak{u}(1): \operatorname{tr}(X)=2 t\} \simeq \mathfrak{u}(n) .
$$

Let $\mathfrak{a}_{0}$ be the diagonal Cartan subalgebra of $\mathfrak{g}_{0}$, and let $A=\exp \left(\mathfrak{a}_{0}\right)$. Here exp denotes the exponential map in $M p(2 n)$. The restricted roots

$$
\Delta\left(\mathfrak{g}_{0}, \mathfrak{a}_{0}\right)=\left\{ \pm \epsilon_{k} \pm \epsilon_{l}\right\}_{1 \leq k<l \leq n} \cup\left\{ \pm 2 \epsilon_{k}\right\}_{k=1, \ldots, n}
$$

form a root system of type $C_{n}$, which will be denoted by $\Delta$. The Weyl group $W=W(\Delta)$ can be realized as $N_{K}(A) / Z_{K}(A)$. It is isomorphic to $S_{n} \ltimes(\mathbb{Z} / 2 \mathbb{Z})^{n}$ and consists of all permutations and sign changes on $n$ coordinates.

3.1. The Groups $G_{\alpha}$. For each root $\alpha \in \Delta$ we choose a Lie algebra homomorphism

$$
\phi_{\alpha}: \mathfrak{s l}(2, \mathbb{R}) \rightarrow \mathfrak{g}_{0}=\mathfrak{s p}(2 n, \mathbb{R})
$$

as in (4.3.6) of 21. The image of $\phi_{\alpha}$ is a subalgebra of $\mathfrak{g}_{0}$ isomorphic to $\mathfrak{s l}(2, \mathbb{R})$. The corresponding connected subgroups of $S p(2 n, \mathbb{R})$ and $M p(2 n)$ will be denoted by $G_{\alpha}^{L}$ and $G_{\alpha}$, respectively. Notice that $G_{\alpha}^{L}$ is always isomorphic to $S L(2, \mathbb{R})$. The group $G_{\alpha}$ is the identity component of the inverse image of $G_{\alpha}^{L}$ under the projection map

$$
\text { pr: } M p(2 n) \rightarrow S p(2 n, \mathbb{R}) ;
$$

hence $G_{\alpha}$ is isomorphic to either $S L(2, \mathbb{R})$ or $M p(2)$. The root $\alpha$ is called "metaplectic" if $G_{\alpha} \simeq M p(2)$, and "non-metaplectic" if $G_{\alpha} \simeq S L(2, \mathbb{R})$. Note that $\alpha$ is metaplectic if and only if it is long (see [1, Theorem 1.6). 
For all $\alpha \in \Delta$, we define:

$$
\begin{aligned}
Z_{\alpha} & :=\phi_{\alpha}\left(\begin{array}{cc}
0 & 1 \\
-1 & 0
\end{array}\right), \\
\sigma_{\alpha} & :=\exp \left(\frac{\pi}{2} Z_{\alpha}\right), \text { and } \\
m_{\alpha} & :=\exp \left(\pi Z_{\alpha}\right)=\sigma_{\alpha}^{2} .
\end{aligned}
$$

The properties of these elements are described in 3. Section 4]. Here we only recall that:

- $Z_{\alpha} \in \mathfrak{k}_{0}$ and generates a subalgebra isomorphic to $\mathfrak{s o}(2)$;

- $\sigma_{\alpha} \in N_{K}(\mathfrak{a})$ and is a representative in $K$ for the root reflection $s_{\alpha}$;

- $m_{\alpha} \in M=Z_{K}(\mathfrak{a})$; it has order at most two if $\alpha$ is non-metaplectic and has order four if $\alpha$ is metaplectic.

Remark 3.1. The homomorphism $\phi_{\alpha}$ is unique up to conjugation of $\mathfrak{s l}(2, \mathbb{R})$ by the diagonal matrix with entries $i$ and $-i$. Hence the element $Z_{\alpha}$ is defined only up to a sign; the elements $\sigma_{\alpha}$ and $m_{\alpha}$ are defined up to inverses. Our choice is recorded in Table 1.

TABLE 1 . The elements $Z_{\alpha}, \sigma_{\alpha}$ and $m_{\alpha}$ associated to a root $\alpha$

\begin{tabular}{|c||c|c|c|}
\hline$\alpha$ & $Z_{\alpha}$ & $\sigma_{\alpha}$ & $m_{\alpha}$ \\
\hline \hline $2 \epsilon_{k}$ & {$\left[i E_{k, k}, \frac{i}{2}\right]$} & {$\left[i E_{k, k}+\sum_{j \neq k} E_{j, j}, e^{\frac{\pi i}{4}}\right]$} & {$\left[-E_{k, k}+\sum_{j \neq k} E_{j, j}, i\right]$} \\
\hline$\epsilon_{k}-\epsilon_{l}$ & {$\left[E_{k, l}-E_{l, k}, 0\right]$} & {$\left[E_{k, l}-E_{l, k}+\sum_{j \neq k, l} E_{j, j}, 1\right]$} & {$\left[-E_{k, k}-E_{l, l}+\sum_{j \neq k, l} E_{j, j}, 1\right]$} \\
\hline$\epsilon_{k}+\epsilon_{l}$ & {$\left[i\left(E_{k, l}+E_{l, k}\right), 0\right]$} & {$\left[i\left(E_{k, l}+E_{l, k}\right)+\sum_{j \neq k, l} E_{j, j}, 1\right]$} & {$\left[-E_{k, k}-E_{l, l}+\sum_{j \neq k, l} E_{j, j}, 1\right]$} \\
\hline
\end{tabular}

Here $E_{j, k}$ is the matrix with all entries 0 , except for the $(j, k)$ entry, which is 1 . 3.2. $M$-types. The centralizer of $A$ in $K$ is denoted by $M$ and consists of all pairs

$$
\left\{[g, z] \in K: g \text { is diagonal with entries } \pm 1 \text {, and } \operatorname{det}(g)=z^{2}\right\} \text {. }
$$

It is an abelian group of order $2^{n+1}$, isomorphic to $\mathbb{Z} / 4 \mathbb{Z} \times(\mathbb{Z} / 2 \mathbb{Z})^{n-1}$. We make the following identifications:

$$
\mathbb{Z} / 4 \mathbb{Z}=\langle[\operatorname{diag}(-1,1,1, \ldots, 1), i]\rangle=\left\langle m_{2 \epsilon_{1}}\right\rangle
$$

and

$$
(\mathbb{Z} / 2 \mathbb{Z})_{j}=\langle[\operatorname{diag}(-1,1, \ldots, 1,-1,1, \ldots, 1), 1]\rangle=\left\langle m_{\epsilon_{1}-\epsilon_{j}}\right\rangle, \text { for } j=2, \ldots, n .
$$

It is more convenient to describe $M$ in terms of the generators

$$
m_{k}:=m_{2 \epsilon_{k}}=[\operatorname{diag}(1, \ldots, 1,-\underset{k}{-1,1}, \ldots, 1), i] \quad k=1, \ldots, n
$$

and the relations

$$
m_{k}^{2}=[I,-1], m_{k} m_{l}=m_{l} m_{k} \quad \forall k, l=1, \ldots, n .
$$


Definition 3.2. A representation of $M$ is called non-genuine if $[I,-1]$ acts trivially, and genuine otherwise.

It is easy to see that the group $M$ admits $2^{n}$ non-genuine one-dimensional representations, in which $[I,-1]$ acts trivially and each $m_{k}$ acts by \pm 1 , and $2^{n}$ genuine one-dimensional representations, in which $[I,-1]$ acts by -1 and each $m_{k}$ acts by $\pm i$. For every subset $S \subset\{1,2, \ldots, n\}$, we denote by $\delta_{S}^{\prime}$ the non-genuine representation satisfying

$$
\delta_{S}^{\prime}\left(m_{k}\right)= \begin{cases}-1 & \text { if } k \in S, \\ +1 & \text { if } k \notin S\end{cases}
$$

for all $k=1, \ldots, n$, and by $\delta_{S}$ the genuine representation satisfying

$$
\delta_{S}\left(m_{k}\right)= \begin{cases}-i & \text { if } k \in S, \\ +i & \text { if } k \notin S\end{cases}
$$

Note that

$$
\delta_{S}^{\prime} \otimes \delta_{T}^{\prime}=\delta_{S \triangle T}^{\prime}, \quad \delta_{S} \otimes \delta_{T}=\delta_{(S \Delta T)^{C}}^{\prime}, \quad \delta_{S} \otimes \delta_{T}^{\prime}=\delta_{S \Delta T} .
$$

(The symbol $(S \triangle T)$ denotes the symmetric difference of two subsets.)

Definition 3.3. A genuine irreducible representation $\delta$ of $M$ is called pseudospherical if -1 is not an eigenvalue of $\delta\left(m_{\alpha}\right)$ for any root $\alpha \in \Delta$.

Remark 3.4. Because

$$
\delta_{S}\left(m_{\epsilon_{k}-\epsilon_{l}}\right)=\delta_{S}\left(m_{2 \epsilon_{k}} m_{2 \epsilon_{l}}^{-1}\right)=-1 \quad \forall k \in S, l \notin S,
$$

there are only two pseudospherical (genuine) $M$-types: $\delta_{\emptyset}$ and $\delta_{\{1, \ldots, n\}}$.

The emphasis of this paper is on genuine representations of $M p(2 n)$. Therefore, we will restrict our attention to genuine $M$-types.

3.3. The stabilizer of a genuine $M$-type. The Weyl group $W$ acts on the set of genuine irreducible representations of $M$ by:

$$
\left(s_{\alpha} \cdot \delta\right)(m):=\delta\left(\sigma_{\alpha}^{-1} m \sigma_{\alpha}\right) \quad \forall m \in M, \forall \alpha \in \Delta .
$$

If $s_{\alpha} \cdot \delta \simeq \delta$, we say that $\alpha$ stabilizes $\delta$. (This condition is equivalent to $s_{\alpha} \cdot \delta=\delta$ because $\delta$ is one-dimensional.) The stabilizer of $\delta$ in $W$ is the subgroup

$$
W^{\delta}:=\{w \in W: w \cdot \delta \simeq \delta\}
$$

of $W$. Note that, for all $1 \leq k<l \leq n$,

$$
s_{2 \epsilon_{k}} \cdot \delta_{S}=\delta_{S} \text { and } s_{\epsilon_{k} \pm \epsilon_{l}} \cdot \delta_{S}= \begin{cases}\delta_{S} & \text { if } k, l \in S \text { or } k, l \in S^{C} \\ \delta_{S \triangle\{k, l\}} & \text { otherwise. }\end{cases}
$$

Therefore, the stabilizer of $\delta_{S}$ is the subgroup of $W$ generated by the reflections across the long roots, together with the reflections across the short roots of the form $\epsilon_{k} \pm \epsilon_{l}$ with $k, l \in S$, or $k, l \in S^{C}$. If $p=\# S^{C}$ and $q=\# S$, this is the Weyl group of a root system of type $C_{p} \times C_{q}$.

Also note that the orbit of $\delta_{S}$ under the Weyl group consists of all characters $\delta_{T}$ with $\# S=\# T$. 
3.4. Good roots. Let $\delta$ be a genuine irreducible representation of $M$. A root $\alpha \in \Delta$ is called "good" for $\delta$ if $\delta\left(m_{\alpha}\right) \neq-1$. Otherwise, we say that $\alpha$ is a "bad" root for $\delta$. The set of good roots for $\delta$ will be denoted by $\Delta_{\delta}$.

Recall that:

$$
\left\{\begin{array}{ll}
m_{2 \epsilon_{k}} & =m_{k}, \\
m_{\epsilon_{k}-\epsilon_{l}} & =m_{k} m_{l}^{3}
\end{array} \quad \text { and } \quad \delta_{S}\left(m_{j}\right)= \begin{cases}+i & \text { if } j \notin S \\
-i & \text { if } j \in S .\end{cases}\right.
$$

Therefore:

$$
\Delta_{\delta_{S}}=\left\{ \pm \epsilon_{k} \pm \epsilon_{l}: k, l \in S \text { or } k, l \in S^{C}\right\} \cup\left\{ \pm 2 \epsilon_{k}: 1 \leq k \leq n\right\} .
$$

If $p=\# S^{C}$ and $q=\# S$, this is a root system of type $C_{p} \times C_{q}$.

Remark 3.5. For every genuine $M$-type $\delta$, the Weyl group of the root system $\Delta_{\delta}$ coincides with the stabilizer of $\delta$, which we have denoted by $W^{\delta}$.

3.5. $K$-types. The equivalence classes of irreducible representations of $K$ are parameterized by highest weights. We will abuse notation and identify each $K$-type with its highest weight. If $\mu=\left(a_{1}, \ldots, a_{n}\right)$ is a $K$-type, the coordinates $a_{i}$ are non-increasing; they belong to $\mathbb{Z}$ if $\mu$ is non-genuine, and to $\mathbb{Z}+\frac{1}{2}$ if $\mu$ is genuine.

Definition 3.6 (cf. [3], Section 4). An irreducible representation $\mu$ of $K$ is

$$
\left\{\begin{array}{l}
\text { pseudospherical } \\
\text { fine } \\
\text { petite }
\end{array} \text { if }|\gamma| \leq\left\{\begin{array}{l}
1 / 2 \\
1 \\
2
\end{array}\right.\right.
$$

respectively, for every root and every eigenvalue $\gamma$ of $\mathrm{d} \mu\left(i Z_{\alpha}\right)$. More generally, we say that a $K$-type is level $k$ if $|\gamma| \leq k$ for every $\alpha$ and every eigenvalue $\gamma$ of $\mathrm{d} \mu\left(i Z_{\alpha}\right)$.

The level of a $K$-type is determined by its highest weight, as follows.

Remark 3.7 ([3], Section 4). Let $\mu$ be a $K$-type with highest weight $\lambda$. Then $\mu$ is level $k$ if and only if

$$
\langle\gamma, \check{\beta}\rangle \leq k
$$

for every imaginary root $\beta$ which is either non-compact long, or compact short.

We deduce that a $K$-type $\left(a_{1}, \ldots, a_{n}\right)$ is petite if and only if

$$
a_{1} \leq 2, \quad a_{n} \geq-2 \text { and } a_{1}-a_{n} \leq 2,
$$

and is fine if and only if

$$
a_{1} \leq 1, \quad a_{n} \geq-1 \quad \text { and } \quad a_{1}-a_{n} \leq 1 .
$$

3.6. Genuine fine $K$-types. The genuine fine $K$-types of $M p(2 n)$, along with their restriction to $M$, are listed in Table 2 .

Remark 3.8. The fine $K$-types $\pm\left(\frac{1}{2}, \ldots, \frac{1}{2}\right)$ are pseudospherical.

We have denoted by $\left\{e_{1}, \ldots, e_{n}\right\}$ the standard basis of $\mathbb{C}^{n}$, and by $\left\{f_{1}, \ldots, f_{n}\right\}$ its dual basis. The symbol $\bigwedge_{j \in S} e_{j}$ denotes the wedge product of the vectors $e_{j}$, with $j \in S$, in, say, increasing order. Setting $p=0$ or $p=n$ in the last row of the table provides an alternative description of the pseudospherical fine $K$-types. 
TABLE 2. Genuine fine $K$-types

\begin{tabular}{|c|c||c|c|}
\hline the fine $K$-type $\mu$ & $\begin{array}{c}\text { an explicit } \\
\text { realization } \\
\text { of } \mu\end{array}$ & $\begin{array}{c}\text { the restriction } \\
\text { of } \mu \text { to } M\end{array}$ & $\begin{array}{c}\text { an explicit } \\
\text { realization } \\
\text { of the } M \text {-types }\end{array}$ \\
\hline \hline \multirow{2}{*}{$\left(\frac{1}{2}, \ldots, \frac{1}{2}\right)$} & $\operatorname{det}^{1 / 2}$ & $\delta_{\emptyset}$ & $\delta_{\emptyset} \leftrightarrow \mathbb{C} 1$ \\
\hline$\left(-\frac{1}{2}, \ldots,-\frac{1}{2}\right)$ & $\operatorname{det}^{-1 / 2}$ & $\delta_{\{1, \ldots, n\}}$ & $\delta_{\{1, \ldots, n\}} \leftrightarrow \mathbb{C} 1$ \\
\hline$(\underbrace{\frac{1}{2}, \ldots, \frac{1}{2}}_{1 \leq p \leq n-1}, \underbrace{-\frac{1}{2}, \ldots,-\frac{1}{2}}_{q})$ & $\Lambda^{p}\left(\mathbb{C}^{n}\right) \otimes \operatorname{det}^{-1 / 2}$ & $\bigoplus_{\# S=q} \delta_{S}$ & $\delta_{S} \leftrightarrow \mathbb{C} \bigwedge_{j \in S^{C}} e_{j}$ \\
\cline { 2 - 4 } & $\Lambda^{q}\left(\mathbb{C}^{n}\right)^{*} \otimes \operatorname{det}^{1 / 2}$ & $\begin{array}{c}\bigoplus_{\# S=q} \delta_{S} \\
\delta_{S} \leftrightarrow \mathbb{C} \bigwedge_{j \in S} f_{j}\end{array}$ \\
\hline
\end{tabular}

Remark 3.9. Note that:

- The restriction of a genuine fine $K$-type to $M$ consists of the $W$-orbit of a single $M$-type. If the $K$-type is pseudospherical, this orbit is onedimensional.

- Every genuine $M$-type is contained in the restriction to $M$ of a (genuine) fine $K$-type. This $K$-type is unique and will be denoted by $\mu_{\delta}$. Note that

$$
\mu_{\delta_{S}}=(\underbrace{\frac{1}{2}, \ldots, \frac{1}{2}}_{p}, \underbrace{-\frac{1}{2}, \ldots,-\frac{1}{2}}_{q})
$$

for all $S$ of cardinality $q$.

3.7. Genuine petite $K$-types. For all indices $a, b, c$ such that $a+b+c=n$, the genuine $K$-type

$$
(\underbrace{\frac{3}{2}, \ldots, \frac{3}{2}}_{a}, \underbrace{\frac{1}{2}, \ldots, \frac{1}{2}}_{b}, \underbrace{-\frac{1}{2}, \ldots,-\frac{1}{2}}_{c})
$$

is petite. We obtain another family of genuine petite $K$-types by dualization.

\section{Genuine complementary series of $M p(2 n)$}

Let $M A$ be the Levi factor of a minimal parabolic subgroup of $G=M p(2 n)$, as in Section 3. Choose a genuine representation $\delta$ of $M$, a real character $\nu$ of $A$ and a minimal parabolic subgroup $P=M A N$ of $G$ making $\nu$ weakly dominant. The induced representation

$$
I_{P}(\delta, \nu):=\operatorname{Ind}_{P}^{G}(\delta \otimes \nu \otimes 1)
$$

is a minimal principal series of $G$.

Remark 4.1. The representation $I_{P}(\delta, \nu)$ is independent of the choice of $P$ (see, e.g., 20, Section 2) and will be denoted by $I(\delta, \nu)$.

Let $\mu_{\delta}$ be the unique fine $K$-type containing $\delta$ (as in Section 3.6). The representation $I(\delta, \nu)$ has a distinguished composition factor containing the fine $K$-type $\mu_{\delta}$, 
which we denote by $J(\delta, \nu)$. We are interested in determining all the parameters $\nu$ that make $J(\delta, \nu)$ unitary.

Notation. Following [3], we write

$$
C S(G, \delta):=\left\{\nu \in \mathfrak{a}_{\mathbb{R}}^{*}: J(\delta, \nu) \text { is unitary }\right\}
$$

for the $\delta$-complementary series of $G=M p(2 n)$.

This is a closed set, because unitarity is a closed condition.

Remark 4.2. The $\delta$-complementary series of $G$ is a $W^{\delta}$-invariant set.

Proof. Recall that $J(\delta, \nu) \simeq J(w \cdot \delta, w \cdot \nu)$ for all $w \in W$. If $w$ stabilizes $\delta$, then $J(\delta, \nu)$ is unitary if and only if $J(\delta, w \cdot \nu)$ is unitary.

As an example, we consider the non-pseudospherical genuine principal series of $M p(6)$. For $M p(6)$, the group $M$ has six inequivalent non-pseudospherical genuine representations; these $M$-types fall into two Weyl group orbits, each consisting of three representations. Note that it is sufficient to consider a single orbit, because the $M$-types in the other orbit are obtained by duality. Figure 3 shows the $\delta$-complementary series for three non-pseudospherical genuine $M$-types of $M p(6)$ in the same $W$-orbit. Each set is invariant under the action of the appropriate stabilizer, but none of them is invariant under the action of the full Weyl group. Conjugation by an element of $W \backslash W^{\delta}$ permutes the three complementary series.

If $\delta$ is pseudospherical, then the stabilizer of $\delta$ is the entire Weyl group, and the complementary series $C S(G, \delta)$ is a $W$-invariant set. In this case, $C S(G, \delta)$ is completely determined by its restriction to any closed fundamental Weyl chamber: it suffices to fix any minimal parabolic subgroup $P=M A N$, study the unitarity of $J(\delta, \nu)$ for $\nu$ weakly dominant for $N$ and then conjugate this set of (weakly dominant) unitary parameters by $W$ to determine the entire complementary series. The advantage of this construction is that one can use the same choice of $N$ (hence the same intertwining operator) for all $\nu$ of interest.

If $\delta$ is neither spherical nor pseudospherical, then the stabilizer of $\delta$ is a proper subgroup of $W$, and a fundamental domain for the action of $W^{\delta}$ extends beyond a single closed Weyl chamber. Hence, if we fix a minimal parabolic subgroup $P=M A N$, determine the set of unitary parameters that are weakly dominant for $N$, and then conjugate this set by $W^{\delta}$, we will not find the entire complementary series. All the different Weyl chambers in a fundamental domain for the action of $W^{\delta}$ should be considered. Note that each requires a different parabolic subgroup, hence a different intertwining operator. (If we insist on fixing a parabolic subgroup, then all the $M$-types in the $W$-orbit of $\delta$ should be be taken into account.)

4.1. Intertwining operators for genuine principal series. For every element $w$ of the Weyl group, there is a formal intertwining operator

$$
T(w, \delta, \nu): I(\delta, \nu) \rightarrow I(w \delta, w \nu) .
$$

(If $\nu$ is weakly dominant, the operator $T(w, \delta, \nu)$ is analytic in $\nu$, hence well defined.) For each $K$-type $\left(\mu, E_{\mu}\right)$, we obtain an operator

$$
T(w, \mu, \delta, \nu): \operatorname{Hom}_{K}(\mu, I(\delta, \nu)) \rightarrow \operatorname{Hom}_{K}(\mu, I(w \delta, w \nu))
$$

which, by Frobenius reciprocity, can be interpreted as an operator

$$
T(w, \mu, \delta, \nu): \operatorname{Hom}_{M}(\mu, \delta) \rightarrow \operatorname{Hom}_{M}(\mu, w \delta) .
$$




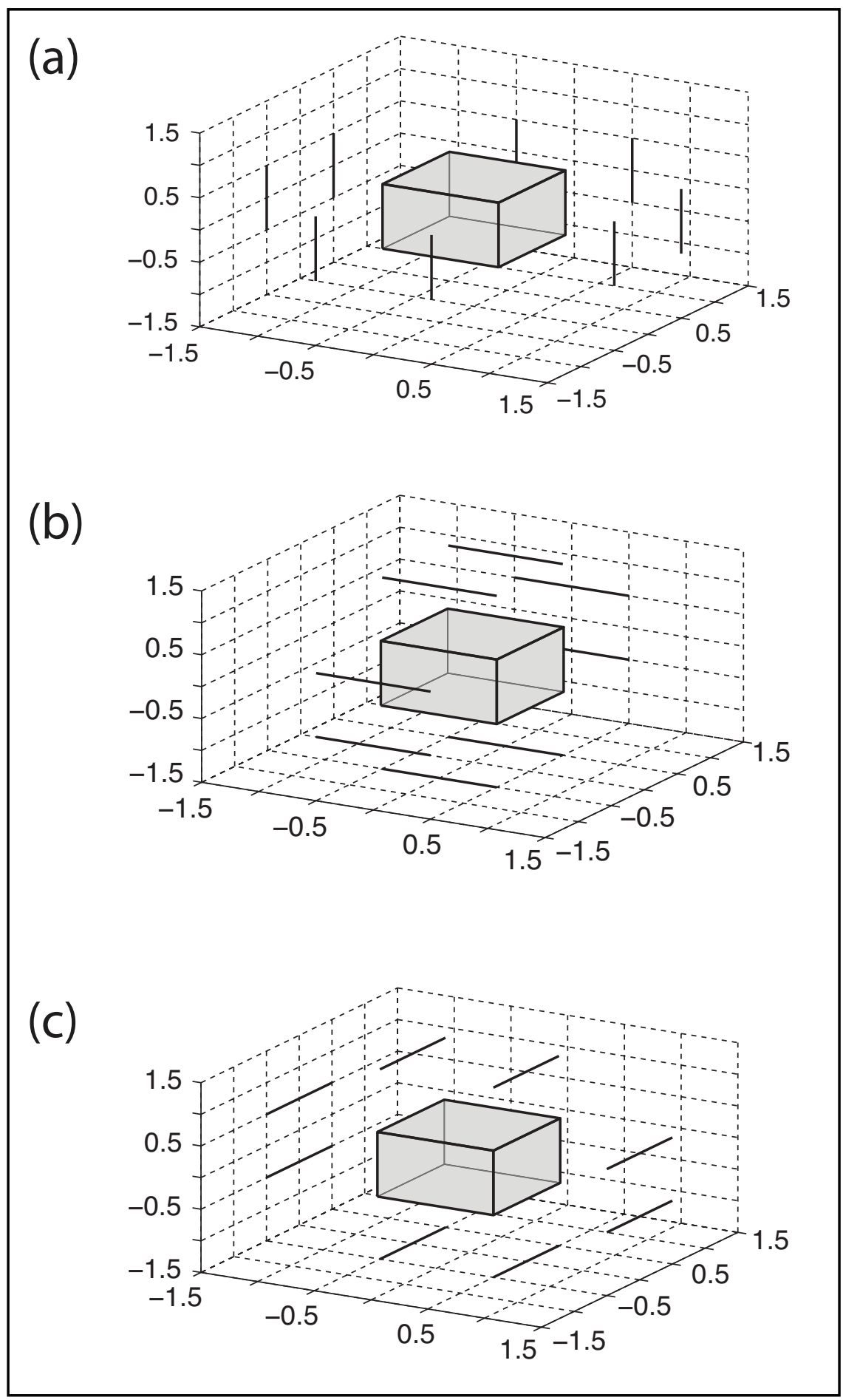

Figure 3. Non-pseudospherical genuine complementary series of $M p(6)$ 
Set $V_{\mu}[\delta]:=\operatorname{Hom}_{M}(\mu, \delta)$, and define $V_{\mu}[w \delta]$ similarly. Because the fine $K$-type $\mu_{\delta}$ contains every $M$-type in the $W$-orbit of $\delta$ with multiplicity one, the operator

$$
T\left(w, \mu_{\delta}, \delta, \nu\right): V_{\mu_{\delta}}[\delta] \rightarrow V_{\mu_{\delta}}[w \delta]
$$

acts by a scalar. We normalize $T(w, \delta, \nu)$ by requiring that this scalar is 1 .

The structure of the space $\operatorname{Hom}_{M}(\mu, \delta)$ is described in detail in Section 5 The action of the operator $T(w, \mu, \delta, \nu)$ on $\operatorname{Hom}_{M}(\mu, \delta)$ is presented in Section 6. Here we only recall the following result.

Proposition. Let $\delta$ be a genuine $M$-type and let $\nu$ be a real character of $A$. Suppose that $\nu$ satisfies:

$$
\begin{cases}\langle\nu, \beta\rangle \notin 2 \mathbb{Z}+1 & \text { for all roots } \beta \text { that are good for } \delta, \\ \langle\nu, \beta\rangle \notin 2 \mathbb{Z} \backslash\{0\} & \text { for all roots } \beta \text { that are bad for } \delta .\end{cases}
$$

Then the operator $T(w, \mu, \delta, \nu)$ has no zero eigenvalues, for every $K$-type $\mu$ containing $\delta$, and for all $w \in W$.

Remark 4.3. If $\delta$ is a pseudospherical irreducible representation of $M$, then every root is good for $\delta$. Condition (4.6) becomes:

$$
\langle\nu, \beta\rangle \notin 2 \mathbb{Z}+1, \forall \beta \in \Delta .
$$

Note that this condition guarantees the invertibility of the spherical intertwining operator with parameter $\nu$ for the real split group $S O(n+1, n)_{0}$ corresponding to the dual root system $\check{\Delta}$ (see Remark 2.3). This is to be expected because, if $\delta$ is pseudospherical, results of [3] tie the unitarity of a pseudospherical Langlands quotient $J(\delta, \nu)$ of $M p(2 n)$ to the unitarity of the spherical Langlands quotient $J\left(\delta_{0}, \nu\right)$ of $S O(n+1, n)_{0}$.

4.2. Unitarity of genuine Langlands quotients. By work of Knapp and Zuckerman, the principal series $I(\delta, \nu)$ (with $\nu$ real) is Hermitian if and only if the long Weyl group element $w_{0}$ satisfies

$$
w_{0} \delta \simeq \delta \text { and } \quad w_{0} \nu=-\nu .
$$

Because $w_{0}=-I d$ for type $C_{n}$, these conditions are always met. Then the principal series $I(\delta, \nu)$ is always Hermitian. The Langlands quotient $J(\delta, \nu)$ inherits an invariant Hermitian form, hence is Hermitian as well.

Assume that $\nu$ is weakly dominant for the positive root system determined by $N$. Then the (formal) operator $T(w, \delta, \nu)$ is analytic in $\nu$, hence well defined, for all $w \in W$. When $w=w_{0}$, the image of $T(w, \delta, \nu)$ is the distinguished composition factor of $I(\delta, \nu)$ containing the fine $K$-type $\mu_{\delta}$, which we have denoted by $J(\delta, \nu)$. The long intertwining operator $T\left(w_{0}, \delta, \nu\right)$ is selfadjoint and defines an invariant Hermitian form on the principal series $I(\delta, \nu)$ :

$$
\langle f, g\rangle=\left(T\left(w_{0}, \delta, \nu\right) f, g\right)_{L^{2}(K)} \quad \forall f, g \in I(\delta, \nu) .
$$

Every space $V_{\mu}[\delta]$ inherits an invariant Hermitian form, via the selfadjoint operator $T\left(w_{0}, \mu, \delta, \nu\right)$. Note that the form on $V_{\mu_{\delta}}[\delta]$ is positive definite, because the operator $T\left(w_{0}, \mu_{\delta}, \delta, \nu\right)$ is trivial.

The (possibly degenerate) invariant form $\langle\cdot, \cdot\rangle$ on $I(\delta, \nu)$ descends to a nondegenerate invariant form on the quotient of $I(\delta, \nu)$ by the kernel of the operator $T\left(w_{0}, \delta, \nu\right)$, which is isomorphic to $J(\delta, \nu)$. The form on $J(\delta, \nu)$ is positive definite 
if and only if $\langle\cdot, \cdot\rangle$ is positive semi-definite. The following theorem asserts that the signature can be computed $K$-type by $K$-type.

Theorem 4.4 (cf. [3]). The Langlands quotient $J(\delta, \nu)$ is unitary if and only if the operator $T\left(w_{0}, \mu, \delta, \nu\right)$ is positive semi-definite, for all $K$-types $\mu$.

Remark 4.5. In the open regions in the complement of the hyperplane arrangement defined by (4.6), the principal series $I(\delta, \nu)$ is irreducible (hence equal to the Langlands quotient $J(\delta, \nu))$, and the operators $T\left(w_{0}, \mu, \delta, \nu\right)$ have constant signature.

Corollary 4.6. The Langlands quotient $J(\delta, \nu)$ is unitary for all $\nu$ in the unit cube:

$$
\left\{\left(x_{1}, \ldots, x_{n}\right) \in \mathfrak{a}_{\mathbb{R}}^{*}|0 \leq| x_{j} \mid \leq \frac{1}{2}, \forall j=1, \ldots, n\right\} .
$$

Proof. Let $P=M A N$ be a minimal parabolic subgroup of $G$ containing our Levi subgroup $M A$ and let $T$ be the closed fundamental Weyl chamber determined by $N$. Every $\nu \in T$ is weakly dominant with respect to the positive root system determined by $N$; hence all the Langlands quotients $J(\delta, \nu)$ with $\nu \in T$ can be constructed using the same parabolic subgroup $P$ (and the same intertwining operator $T\left(w_{0}, \delta, \nu\right)$ ).

Write $T^{\prime}$ for the intersection of $T$ with the interior of the cube. We begin by proving that the Langlands quotient $J(\delta, \nu)$ is unitary for all $\nu \in T^{\prime}$. Because

$$
|\langle\nu, \alpha\rangle|<1 \quad \forall \alpha \in \Delta, \forall \nu \in T^{\prime},
$$

every operator $T\left(w_{0}, \mu, \delta, \nu\right)$ with $\mu \in \widehat{K}$ is invertible on $T^{\prime}$, and has constant signature. When $\nu=0$, the principal series is unitarily induced, hence unitary. We conclude that $T\left(w_{0}, \mu, \delta, \nu\right)$ is positive semidefinite for all $\mu \in \widehat{K}$ and all $\nu \in T^{\prime}$. This proves the unitarity of $J(\delta, \nu)$ for all $\nu \in T^{\prime}$. By varying the choice of $N$, we obtain unitarity for all points in the interior of the cube. The unitarity on the boundary of the cube is automatic, because unitarity is a closed condition.

In Section 7 , we provide a set of strong necessary conditions for the unitarity of genuine Langlands quotients of $M p(2 n)$. A synopsis of the technical background is presented in Sections 5 and 6. Many of the concepts introduced in the next three sections originated in a joint effort (yet unpublished) by D. Barbasch and the first author; cf. [8]. The overarching goal of that program was the development of a broad class of non-unitarity certificates for non-spherical principal series of double covers of real split exceptional groups. The present paper extends those ideas to $M p(2 n)$.

\section{The SPACE $\operatorname{Hom}_{M}(\mu, \delta)$}

5.1. An action of the stabilizer of $\delta$ on the space $\operatorname{Hom}_{M}(\mu, \delta)$. Let $\left(\delta, V^{\delta}\right)$ be an $M$-type, and let $\mu_{\delta}$ be a fixed fine $K$-type containing $\delta$. (The choice of $\mu_{\delta}$ is unique for $\delta$ genuine.) For every $K$-type $\left(\mu, E_{\mu}\right)$ containing $\delta$, we define a representation $\psi_{\mu}$ of the stabilizer $W^{\delta}$ of $\delta$ on the space $V_{\mu}[\delta]:=\operatorname{Hom}_{M}(\mu, \delta)$, as follows. Because $\delta$ appears in $\mu_{\delta}$ with multiplicity one, we can identify the representation $\delta$ with its copy inside $\left.\mu_{\delta}\right|_{M}$, and the space $V^{\delta}$ with the isotypic component $E_{\mu_{\delta}}(\delta)$ of $\delta$ in $\mu_{\delta}$. Let $M_{\delta}^{\prime}$ be the preimage of $W^{\delta}$ in the normalizer $M^{\prime}$ of $A$ in $K$, so that $W^{\delta}=M_{\delta}^{\prime} / M$. The group $M_{\delta}^{\prime}$ acts on both $E_{\mu}(\delta)$ and $E_{\mu_{\delta}}(\delta)$ by restriction of the appropriate action of $K$; hence it acts on

$$
V_{\mu}[\delta]=\operatorname{Hom}_{M}(\mu, \delta)=\operatorname{Hom}_{M}\left(E_{\mu}, E_{\mu_{\delta}}(\delta)\right)=\operatorname{Hom}_{M}\left(E_{\mu}(\delta), E_{\mu_{\delta}}(\delta)\right)
$$


by

$$
\sigma \cdot T(v)=\mu_{\delta}(\sigma) T\left(\mu(\sigma)^{-1} v\right) \quad \forall v \in E_{\mu}, \sigma \in M_{\delta}^{\prime} .
$$

Since $M$ acts trivially, this action of $M_{\delta}^{\prime}$ factors to a representation of $W^{\delta}=M_{\delta}^{\prime} / M$ on $V_{\mu}[\delta]$, which we denote by $\psi_{\mu}$.

We are interested in the representations that arise from genuine petite $K$-types. It is sufficient to consider only one representative for each $W$-orbit of $M$-types. For each pair of non-negative integers $(p, q)$ such that $p+q=n$, let $\delta_{p, q}$ be the unique genuine representation of $M$ satisfying:

$$
\delta_{p, q}\left(m_{2 \epsilon_{k}}\right)= \begin{cases}+i & \text { if } k \leq p, \\ -i & \text { if } k>p .\end{cases}
$$

(In the notation of Section 3.2, $\delta_{p, q}=\delta_{\{p+1, \ldots, n\}}$. ) Following [3], we call

$$
\Sigma_{K}\left(G, \delta_{p, q}\right):=\left\{\mu \in \widehat{K} \mid \mu \text { is petite, and }\left.\mu\right|_{M} \supset \delta_{p, q}\right\}
$$

the set of irreducible petite representations of $K$ containing $\delta_{p, q}$, and

$$
\Sigma_{W}\left(G, \delta_{p, q}\right):=\left\{\psi \in\left(\widehat{W^{\delta_{p, q}}}\right) \mid \psi \subset \psi_{\mu} \text { for some } \mu \in \Sigma_{K}\left(G, \delta_{p, q}\right)\right\}
$$

the set of irreducible representations of $W^{\delta_{p, q}} \simeq W\left(C_{p}\right) \times W\left(C_{q}\right)$ that can be realized on the space $V_{\mu}\left[\delta_{p, q}\right]$ for some petite $K$-type $\mu$.

Recall the notion of relevant representation from Theorem 2.8 .

Proposition 5.1. The set $\Sigma_{W}\left(G, \delta_{p, q}\right)$ contains every irreducible relevant representation of $W\left(C_{p}\right)$ and every irreducible relevant representation of $W\left(C_{q}\right)$. The precise matching is recorded in Table 3 .

TABLE 3. Matching of petite $K$-types and relevant $W^{\delta_{p, q}}$-types

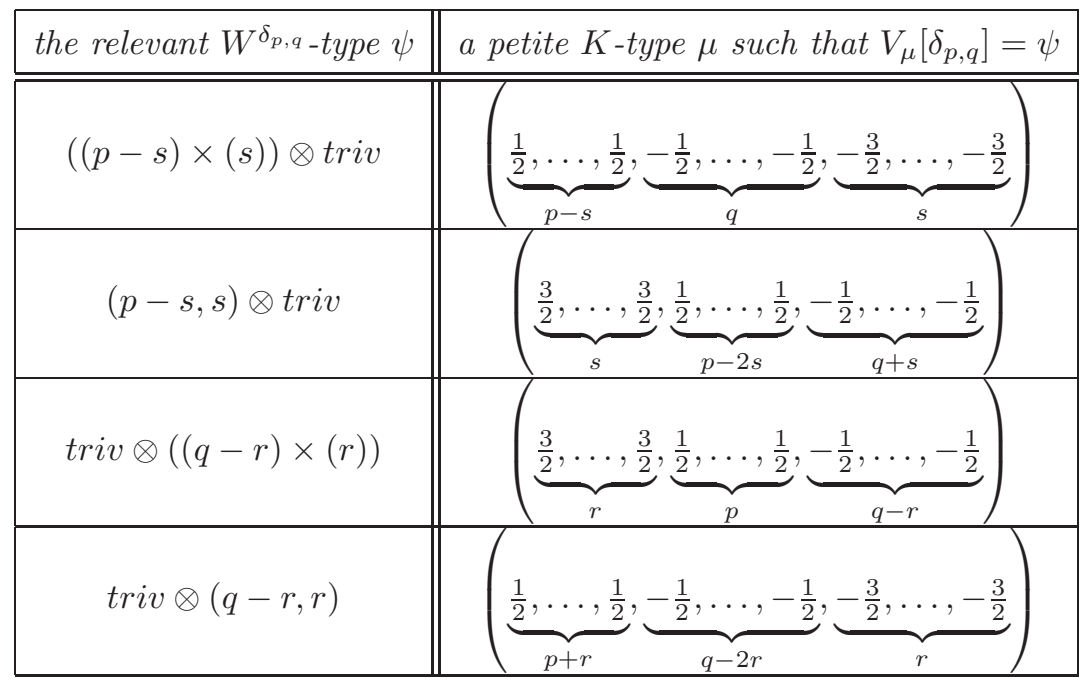

The proof of Proposition 5.1 is given in Section 10 
5.2. The structure of the space $V_{\mu}[\delta]$. For each root $\alpha \in \Delta$, we define an action of $\left(Z_{\alpha}\right)^{2}$ on $E_{\mu}$ via $\left(\mathrm{d} \mu\left(Z_{\alpha}\right)\right)^{2}$. The decomposition of $E_{\mu}$ into $\left(Z_{\alpha}\right)^{2}$-generalized eigenspaces induces a decomposition of the space

$$
V_{\mu}[\delta]:=\operatorname{Hom}_{M}(\mu, \delta) .
$$

If $\mu$ is petite and $\alpha$ is good for $\delta$, this decomposition coincides with the decomposition of $V_{\mu}[\delta]$ into $( \pm 1)$-eigenspaces for the action of $\psi_{\mu}\left(s_{\alpha}\right)$, with $\psi_{\mu}$ the representation of $W^{\delta}$ on $V_{\mu}[\delta]$ introduced in Section 5.1.

5.2.1. An action of $\left(Z_{\alpha}\right)^{2}$ on $E_{\mu}$. Let $\delta$ be a genuine irreducible representation of $M$ and let $\left(\mu, E_{\mu}\right)$ be a $K$-type containing $\delta$. For each root $\alpha$, we choose an element $Z_{\alpha} \in \mathfrak{k}$ as in Section 3.1. Recall that:

(1) $Z_{\alpha}$ generates a subalgebra isomorphic to $\mathfrak{s o}(2)$.

(2) $\sigma_{\alpha}:=\exp \left(\frac{\pi}{2} Z_{\alpha}\right)$ is a representative in $K$ for the root reflection $s_{\alpha}$.

(3) $m_{\alpha}:=\exp \left(\pi Z_{\alpha}\right)$ belongs to $M$ and satisfies $m_{\alpha}^{2}=[I,-1]$ if $\alpha$ is metaplectic and $m_{\alpha}^{2}=[I, 1]$ otherwise.

Because

$$
\exp \left(4 \pi \mathrm{d} \mu\left(Z_{\alpha}\right)\right)=\mu\left(m_{\alpha}^{4}\right)=I d,
$$

the element $\left(i Z_{\alpha}\right)$ acts on $E_{\mu}$ with half-integer eigenvalues. (The eigenvalues lie in $\mathbb{Z}+\frac{1}{2}$ if $\alpha$ is metaplectic, and in $\mathbb{Z}$ otherwise.) For all $k \in \mathbb{Z} / 2$, let $V_{\mu}^{\alpha}(k)$ be the generalized eigenspace of $\mathrm{d} \mu\left(i Z_{\alpha}\right)$ with eigenvalue $k$ :

$$
V_{\mu}^{\alpha}(k):=\left\{v \in E_{\mu}: \mathrm{d} \mu\left(i Z_{\alpha}\right) v=k v\right\} .
$$

The space

$$
V_{\mu}^{\alpha}( \pm k):=V_{\mu}^{\alpha}(k)+V_{\mu}^{\alpha}(-k)
$$

is stable under the action of $M$ (because $A d(m) Z_{\alpha}= \pm Z_{\alpha}, \forall m \in M$ ), and it coincides with the generalized eigenspace of eigenvalue $\left(-k^{2}\right)$ for the action of $Z_{\alpha}^{2}$ on $E_{\mu}$ via $\left(\mathrm{d} \mu\left(Z_{\alpha}\right)\right)^{2}$. Therefore, we can write:

$$
V_{\mu}[\delta]=\operatorname{Hom}_{M}\left(E_{\mu}, \delta\right)= \begin{cases}\bigoplus_{k \in \mathbb{N}+\frac{1}{2}} \operatorname{Hom}_{M}\left(V_{\mu}^{\alpha}( \pm k), \delta\right) & \text { if } \alpha \text { is metaplectic } \\ \bigoplus_{k \in \mathbb{N}} \operatorname{Hom}_{M}\left(V_{\mu}^{\alpha}( \pm k), \delta\right) & \text { otherwise }\end{cases}
$$

In case the index $k$ is an integer, its parity is dictated by the following remark.

Remark 5.2. Let $E_{\mu}(\delta)$ be the isotypic component of $\delta$ inside $\mu$. If $\alpha$ is nonmetaplectic, the eigenvalues of $\left(\mathrm{d} \mu\left(Z_{\alpha}\right)\right)^{2}$ on $E_{\mu}(\delta)$ are all even integers if $\delta\left(m_{\alpha}\right)=$ 1 , and all odd integers if $\delta\left(m_{\alpha}\right)=-1$.

For further applications, it is convenient to have an explicit description of the actions of $\sigma_{\alpha}=\exp \left(\frac{\pi}{2} Z_{\alpha}\right)$ and $m_{\alpha}=\exp \left(\pi Z_{\alpha}\right)$ on the space $V_{\mu}^{\alpha}( \pm k) \cap E_{\mu}(\delta)$. See Table 4 . 
TABLE 4. Actions of $\sigma_{\alpha}$ and $m_{\alpha}$ on the space $V_{\mu}^{\alpha}( \pm k) \cap E_{\mu}(\delta)$

\begin{tabular}{|c||c|c|c|}
\hline$v \in V_{\mu}^{\alpha}( \pm k) \cap E_{\mu}(\delta)$ & $\mathrm{d} \mu\left(Z_{\alpha}\right)^{2} v$ & $\mu\left(\sigma_{\alpha}\right) v$ & $\delta\left(m_{\alpha}\right) v$ \\
\hline \hline$k=0$ & 0 & $+v$ & $+v$ \\
\hline$k=2 n$ & $-(2 n)^{2} v$ & $(-1)^{n} v$ & $+v$ \\
\hline$k=2 n+1$ & $-(2 n+1)^{2} v$ & $\frac{1}{2 n+1}(-1)^{n} \mathrm{~d} \mu\left(Z_{\alpha}\right) v$ & $-v$ \\
\hline$k=2 n+\frac{1}{2}$ & $-\left(2 n+\frac{1}{2}\right)^{2} v$ & $e^{\left(2 n+\frac{1}{2}\right) \frac{\pi}{2} i} v$ & $+i v$ \\
\cline { 3 - 4 } & & $e^{-\left(2 n+\frac{1}{2}\right) \frac{\pi}{2} i} v$ & $-i v$ \\
\hline$k=2 n+\frac{3}{2}$ & \multirow{2}{*}{$-\left(2 n+\frac{3}{2}\right)^{2} v$} & $e^{-\left(2 n+\frac{3}{2}\right) \frac{\pi}{2} i} v$ & $+i v$ \\
\cline { 3 - 4 } & & $e^{\left(2 n+\frac{3}{2}\right) \frac{\pi}{2} i} v$ & $-i v$ \\
\hline
\end{tabular}

To prove these formulas, we observe that if $v$ is a non-zero vector in $V_{\mu}^{\alpha}( \pm k) \cap$ $E_{\mu}(\delta)$ and $k \neq 0$, then

$$
\begin{aligned}
& \mu\left(\exp \left(t Z_{\alpha}\right)\right) v=\exp \left(t \mathrm{~d} \mu\left(Z_{\alpha}\right)\right) v=\sum_{n=0}^{\infty} \frac{1}{n !} t^{n}\left[\mathrm{~d} \mu\left(Z_{\alpha}\right)\right]^{n} v \\
& =\sum_{n=0}^{\infty} \frac{1}{(2 n) !} t^{2 n} \underbrace{\left[\mathrm{d} \mu\left(Z_{\alpha}\right)\right]^{2 n} v}_{\left(-k^{2}\right)^{n} v}+\sum_{n=0}^{\infty} \frac{1}{(2 n+1) !} t^{2 n+1} \underbrace{\left[\mathrm{d} \mu\left(Z_{\alpha}\right)\right]^{2 n+1} v}_{\left(-k^{2}\right)^{n} \mathrm{~d} \mu\left(Z_{\alpha}\right) v} \\
& =\left[\sum_{n=0}^{\infty} \frac{1}{(2 n) !}(-1)^{n}(k t)^{2 n}\right] v+\frac{1}{k}\left[\sum_{n=0}^{\infty} \frac{1}{(2 n+1) !}(-1)^{n}(k t)^{2 n+1}\right] \mathrm{d} \mu\left(Z_{\alpha}\right) v \\
& =\cos (k t) v+\frac{1}{k} \sin (k t) \mathrm{d} \mu\left(Z_{\alpha}\right) v
\end{aligned}
$$

for all $t \in \mathbb{R}$. For $t=\pi / 2$ and $t=\pi$, we obtain:

$$
\left\{\begin{aligned}
\mu\left(\sigma_{\alpha}\right) v & =\cos \left(\frac{\pi}{2} k\right) v+\frac{1}{k} \sin \left(\frac{\pi}{2} k\right) \mathrm{d} \mu\left(Z_{\alpha}\right) v \\
\mu\left(m_{\alpha}\right) v & =\cos (\pi k) v+\frac{1}{k} \sin (\pi k) \mathrm{d} \mu\left(Z_{\alpha}\right) v .
\end{aligned}\right.
$$

If $k=0$, the same calculation shows that $\mu\left(\exp \left(t Z_{\alpha}\right)\right) v=v+t \mathrm{~d} \mu\left(Z_{\alpha}\right) v$.

Remark 5.3. Two different eigenvalues for $Z_{\alpha}$ in $E_{\mu}$ must differ by an even integral multiple of $i$; therefore, if $k \in \mathbb{Z}+\frac{1}{2}$, then at most one of $V_{\mu}^{\alpha}( \pm k) \cap E_{\mu}(\delta)$ can be non-zero. Consequently, we have that if $\alpha$ is a good root for $\delta$, i.e., if $\delta\left(m_{\alpha}\right) \neq-1$, then $\sigma_{\alpha}$ acts on each space $V_{\mu}^{\alpha}( \pm k) \cap E_{\mu}(\delta)$ by a scalar.

5.3. Genuine petite $K$-types. Let $\left(\mu, E_{\mu}\right)$ be a petite $K$-type containing $\delta$ and let $\alpha$ be a good root for $\delta$. The action of $\left(Z_{\alpha}\right)^{2}$ on $E_{\mu}$ induces a decomposition of the space $V_{\mu}[\delta]$, as in equation (5.9). We claim that this is also the decomposition of $V_{\mu}[\delta]$ as a direct sum of $( \pm 1)$-eigenspaces for $\psi_{\mu}\left(s_{\alpha}\right)$. Precisely:

$$
V_{\mu}[\delta]=\underbrace{\operatorname{Hom}_{M}\left(V_{\mu}^{\alpha}( \pm 1 / 2), \delta\right)}_{(+1) \text {-eigenspace of } \psi_{\mu}\left(s_{\alpha}\right)} \oplus \underbrace{\operatorname{Hom}_{M}\left(V_{\mu}^{\alpha}( \pm 3 / 2), \delta\right)}_{(-1) \text {-eigenspace of } \psi_{\mu}\left(s_{\alpha}\right)}
$$


if $\alpha$ is metaplectic, and

$$
V_{\mu}[\delta]=\underbrace{\operatorname{Hom}_{M}\left(V_{\mu}^{\alpha}(0), \delta\right)}_{(+1) \text {-eigenspace of } \psi_{\mu}\left(s_{\alpha}\right)} \oplus \underbrace{\operatorname{Hom}_{M}\left(V_{\mu}^{\alpha}( \pm 2), \delta\right)}_{(-1) \text {-eigenspace of } \psi_{\mu}\left(s_{\alpha}\right)}
$$

if $\alpha$ is non-metaplectic.

We give a proof by direct computation. Identify $V_{\mu}[\delta]$ with the space

$$
\operatorname{Hom}_{M}\left(E_{\mu}(\delta), E_{\mu_{\delta}}(\delta)\right)
$$

and define

$$
\left(\psi_{\mu}\left(s_{\alpha}\right) T\right)(v)=\mu_{\delta}\left(\sigma_{\alpha}\right) T\left(\mu\left(\sigma_{\alpha}^{-1}\right) v\right) \quad \forall T \in V_{\mu}[\delta], v \in E_{\mu}(\delta),
$$

as in Section 5.1, Then

$$
E_{\mu}(\delta)= \begin{cases}{\left[E_{\mu}(\delta) \cap V_{\mu}^{\alpha}( \pm 1 / 2)\right] \oplus\left[E_{\mu}(\delta) \cap V_{\mu}^{\alpha}( \pm 3 / 2)\right]} & \text { if } \alpha \text { is metaplectic } \\ {\left[E_{\mu}(\delta) \cap V_{\mu}^{\alpha}(0)\right] \oplus\left[E_{\mu}(\delta) \cap V_{\mu}^{\alpha}( \pm 2)\right]} & \text { otherwise }\end{cases}
$$

because $\mu$ has level at most two, and

$$
E_{\mu_{\delta}}(\delta)= \begin{cases}E_{\mu_{\delta}}(\delta) \cap V_{\mu_{\delta}}^{\alpha}( \pm 1 / 2) & \text { if } \alpha \text { is metaplectic, } \\ E_{\mu_{\delta}}(\delta) \cap V_{\mu_{\delta}}^{\alpha}(0) & \text { otherwise }\end{cases}
$$

because $\mu_{\delta}$ is fine. The action of $\sigma_{\alpha}$ on these spaces is recorded in Table 5 .

TABLE 5

\begin{tabular}{|c||c|}
\hline & $\mu\left(\sigma_{\alpha}\right)$ \\
\hline \hline$E_{\mu}(\delta) \cap V_{\mu}^{\alpha}( \pm 0)$ & +1 \\
\hline$E_{\mu}(\delta) \cap V_{\mu}^{\alpha}( \pm 2)$ & -1 \\
\hline$E_{\mu}(\delta) \cap V_{\mu}^{\alpha}( \pm 1 / 2)$ & $e^{\frac{\pi}{4} \varepsilon i}$ \\
\hline$E_{\mu}(\delta) \cap V_{\mu}^{\alpha}( \pm 3 / 2)$ & $e^{\frac{3 \pi}{4} \varepsilon i}$ \\
\hline
\end{tabular}

\begin{tabular}{|c||c|}
\hline & $\mu_{\delta}\left(\sigma_{\alpha}\right)$ \\
\hline \hline$E_{\mu_{\delta}}(\delta) \cap V_{\mu_{\delta}}^{\alpha}( \pm 0)$ & 1 \\
\hline$E_{\mu_{\delta}}(\delta) \cap V_{\mu_{\delta}}^{\alpha}( \pm 1 / 2)$ & $e^{\frac{\pi}{4} \varepsilon i}$ \\
\hline
\end{tabular}

Here $\varepsilon= \pm 1$ (depending on $\delta\left(m_{\alpha}\right)$ ). The claim follows.

\section{IntertWINING OPERATORS FOR GENUINE PRINCIPAL SERIES OF $M p(2 n)$}

Let $\delta$ be a genuine $M$-type. We describe the formal intertwining operator $T(w, \mu, \delta, \nu)$ introduced in Section 4 . The reader can consult [14 for more details.

Remark 6.1 . We can identify every $M$-type in the $W$-orbit of $\delta$ with its unique copy inside the fine $K$-type $\mu_{\delta}$, and we can think of the operator

$$
T(w, \mu, \delta, \nu): V_{\mu}[\delta] \rightarrow V_{\mu}[w \delta]
$$

as an endomorphism of the space $\operatorname{Hom}_{M}\left(\mu, \mu_{\delta}\right)$.

Let

$$
w=s_{\alpha_{r}} s_{\alpha_{r-1}} \cdots s_{\alpha_{1}}
$$

be a minimal decomposition of $w$ as a product of simple reflections. The operator $T(w, \mu, \delta, \nu)$ factors as a product of operators of the form

$$
T\left(s_{\alpha}, \mu, \tau, \gamma\right): V_{\mu}[\tau] \rightarrow V_{\mu}\left[s_{\alpha} \tau\right]
$$


with $\tau$ an $M$-type in the $W$-orbit of $\delta$, and $\gamma$ an element of $\mathfrak{a}_{\mathbb{R}}^{*}$. Precisely:

$$
T(w, \mu, \delta, \nu)=\prod_{j=1}^{r} T\left(s_{\alpha_{j}}, \mu, \delta_{j-1}, \nu_{j-1}\right)
$$

with $\delta_{0}=\delta, \nu_{0}=\nu$, and

$$
\delta_{j}=\underbrace{s_{\alpha_{j}} s_{\alpha_{j-1}} \cdots s_{\alpha_{1}}}_{x_{j}} \cdot \delta=x_{j} \cdot \delta, \quad \nu_{j}=\underbrace{s_{\alpha_{j}} s_{\alpha_{j-1}} \cdots s_{\alpha_{1}}}_{x_{j}} \cdot \nu=x_{j} \cdot \nu
$$

for $j \geq 1$. We show how to compute a factor $T\left(s_{\alpha}, \mu, \tau, \gamma\right)$ of this decomposition. Recall from Section 5.2.1 that the action of $\left(\mathrm{d} \mu\left(Z_{\alpha}\right)\right)^{2}$ on $E_{\mu}$ induces a decomposition of the $\operatorname{Hom}_{M}$-spaces. Write:

$$
V_{\mu}[\tau]= \begin{cases}\bigoplus_{k \in 2 \mathbb{N}} \operatorname{Hom}_{M}\left(V_{\mu}^{\alpha}( \pm k), \tau\right) & \text { if } \alpha \text { is non-metaplectic and good for } \tau \\ \bigoplus_{k \in 2 \mathbb{N}+1} \operatorname{Hom}_{M}\left(V_{\mu}^{\alpha}( \pm k), \tau\right) & \text { if } \alpha \text { is non-metaplectic and bad for } \tau \\ \bigoplus_{k \in \mathbb{N}+1 / 2} \operatorname{Hom}_{M}\left(V_{\mu}^{\alpha}( \pm k), \tau\right) & \text { if } \alpha \text { is metaplectic }\end{cases}
$$

and decompose $V_{\mu}\left[s_{\alpha} \tau\right]$ similarly. The operator

$$
T\left(s_{\alpha}, \mu, \tau, \gamma\right): V_{\mu}[\tau] \rightarrow V_{\mu}\left[s_{\alpha} \tau\right]
$$

maps

$$
\operatorname{Hom}_{M}\left(V_{\mu}^{\alpha}( \pm k), \tau\right) \rightarrow \operatorname{Hom}_{M}\left(V_{\mu}^{\alpha}( \pm k), s_{\alpha} \tau\right)
$$

for all $k \in \mathbb{N} / 2$, via

$$
F \mapsto d_{k}(\alpha, \gamma) \mu_{\delta}\left(\sigma_{\alpha}\right)\left(F \circ \mu\left(\sigma_{\alpha}^{-1}\right)\right) .
$$

The constant $d_{k}(\alpha, \gamma)$ depends on the half-integer $k$ and on the inner product $\lambda=\langle\gamma, \check{\alpha}\rangle$. Precisely, we have:

$$
d_{0}(\alpha, \gamma)=d_{\frac{1}{2}}(\alpha, \gamma)=d_{1}(\alpha, \gamma)=1
$$

and

$$
\begin{aligned}
d_{\frac{3}{2}+2 m}(\alpha, \gamma) & =(-1)^{m+1} \frac{\left(\frac{1}{2}-\lambda\right)\left(\frac{5}{2}-\lambda\right) \cdots\left(\frac{1}{2}+2 m-\lambda\right)}{\left(\frac{1}{2}+\lambda\right)\left(\frac{5}{2}+\lambda\right) \cdots\left(\frac{1}{2}+2 m+\lambda\right)}, \\
d_{\frac{5}{2}+2 m}(\alpha, \gamma) & =(-1)^{m+1} \frac{\left(\frac{3}{2}-\lambda\right)\left(\frac{7}{2}-\lambda\right) \cdots\left(\frac{3}{2}+2 m-\lambda\right)}{\left(\frac{3}{2}+\lambda\right)\left(\frac{7}{2}+\lambda\right) \cdots\left(\frac{3}{2}+2 m+\lambda\right)}, \\
d_{2 m+1}(\alpha, \gamma) & =(-1)^{m} \frac{(2-\lambda)(4-\lambda) \cdots(2 m-\lambda)}{(2+\lambda)(4+\lambda) \cdots(2 m+\lambda)}, \\
d_{2 m}(\alpha, \gamma) & =(-1)^{m} \frac{(1-\lambda)(3-\lambda) \cdots(2 m-1-\lambda)}{(1+\lambda)(3+\lambda) \cdots(2 m-1+\lambda)}
\end{aligned}
$$

for all $m>0$.

If the root $\alpha$ is good for $\tau$, then the operator $T\left(s_{\alpha}, \mu, \tau, \gamma\right)$ has a simpler form.

Proposition 6.2. If $\alpha$ is a good root for $\tau$, then the reflection $s_{\alpha}$ stabilizes $\tau$ and

$$
T\left(s_{\alpha}, \mu, \tau, \gamma\right): V_{\mu}[\tau] \rightarrow V_{\mu}[\tau]
$$


is an endomorphism of $V_{\mu}[\tau]$. It acts on each subspace $\operatorname{Hom}_{M}\left(V_{\mu}^{\alpha}( \pm k), \tau\right)$ by the scalar

$$
b_{k}(\alpha, \gamma):=\left\{\begin{array}{cl}
1 & \text { if } k=0 \text { or } 1 / 2, \\
\prod_{0 \leq j \leq[(k-1) / 2]} \frac{(k-1-2 j)-\langle\gamma, \check{\alpha}\rangle}{(k-1-2 j)+\langle\gamma, \check{\alpha}\rangle} & \text { if } k>1 / 2 .
\end{array}\right.
$$

Proof. By assumption, $\tau$ is an $M$-type in the $W$-orbit of $\delta$. Hence we can identify $\tau$ with its copy inside the fine $K$-type $\mu_{\delta}$, and write:

$$
\left.\mu_{\delta}\left(\sigma_{\alpha}\right)\right|_{E_{\mu_{\delta}}(\tau)}= \begin{cases}1 & \text { if } \tau\left(m_{\alpha}\right)=1 \\ e^{\varepsilon \frac{\pi}{4} i} & \text { if } \tau\left(m_{\alpha}\right)=\varepsilon i\end{cases}
$$

with $\varepsilon= \pm 1$. Recall from Section 5.2 .1 that

$$
\left.\mu\left(\sigma_{\alpha}\right)\right|_{V_{\mu}^{\alpha}( \pm k) \cap E_{\mu}(\tau)}= \begin{cases}(-1)^{n} & \text { if } k=2 n \text { and } \tau\left(m_{\alpha}\right)=1, \\ e^{\varepsilon\left(2 n+\frac{1}{2}\right) \frac{\pi}{2} i} & \text { if } k=2 n+\frac{1}{2} \text { and } \tau\left(m_{\alpha}\right)=\varepsilon i, \\ e^{-\varepsilon\left(2 n+\frac{3}{2}\right) \frac{\pi}{2} i} & \text { if } k=2 n+\frac{3}{2} \text { and } \tau\left(m_{\alpha}\right)=\varepsilon i .\end{cases}
$$

The action of $T\left(s_{\alpha}, \mu, \tau, \gamma\right)$ on an element $T \in \operatorname{Hom}_{M}\left(V_{\mu}^{\alpha}( \pm k), \tau\right)$ is then given by:

$$
\begin{aligned}
& T\left(s_{\alpha}, \mu, \tau, \gamma\right) \cdot T=d_{k}(\alpha, \gamma) \mu_{\delta}\left(\sigma_{\alpha}\right)\left(T \circ \mu\left(\sigma_{\alpha}^{-1}\right)\right) \\
& \quad= \begin{cases}(-1)^{n} d_{k}(\alpha, \gamma) T & \text { if } k=2 n, \\
e^{-\varepsilon\left(2 n+\frac{1}{2}\right) \frac{\pi}{2} i} e^{\varepsilon \frac{\pi}{4} i} d_{k}(\alpha, \gamma) T=(-1)^{n} d_{k}(\alpha, \gamma) T & \text { if } k=2 n+\frac{1}{2}, \\
e^{+\varepsilon\left(2 n+\frac{3}{2}\right) \frac{\pi}{2} i} e^{\varepsilon \frac{\pi}{4} i} d_{k}(\alpha, \gamma) T=(-1)^{n+1} d_{k}(\alpha, \gamma) T & \text { if } k=2 n+\frac{3}{2} .\end{cases}
\end{aligned}
$$

This proves the claim because

$$
b_{k}(\alpha, \gamma)= \begin{cases}(-1)^{\frac{k}{2}} d_{k}(\alpha, \gamma) & \text { if } k \in 2 \mathbb{N} \\ (-1)^{\frac{k+1 / 2}{2}} d_{k}(\alpha, \gamma) & \text { if } k \in \frac{3}{2}+2 \mathbb{N} \\ (-1)^{\frac{k-1 / 2}{2}} d_{k}(\alpha, \gamma) & \text { if } k \in \frac{5}{2}+2 \mathbb{N}\end{cases}
$$

Remark 6.3. Suppose that for every positive root $\alpha,\langle\alpha, \nu\rangle$ is not a non-negative integer. Then the (formal) operator $T(w, \delta, \nu)$ is analytic in $\nu$, hence well defined.

We now discuss the invertibility of the operator $T(w, \mu, \delta, \nu)$.

Proposition 6.4. Choose a minimal decomposition of $w$ in $W$ :

$$
w=s_{\alpha_{r}} s_{\alpha_{r-1}} \cdots s_{\alpha_{1}}
$$

and write $x_{j}:=s_{\alpha_{j}} s_{\alpha_{j-1}} \cdots s_{\alpha_{1}}$ for all $j=1, \ldots, r$, and $x_{0}=1$. Suppose that

$$
\begin{cases}\left\langle\nu, x_{j-1}^{-1} \cdot \alpha_{j}\right\rangle \notin 2 \mathbb{N}+1 & \text { if } x_{j-1}^{-1} \cdot \alpha_{j} \text { is good for } \delta \\ \left\langle\nu, x_{j-1}^{-1} \cdot \alpha_{j}\right\rangle \notin 2 \mathbb{N}+2 & \text { if } x_{j-1}^{-1} \cdot \alpha_{j} \text { is bad for } \delta\end{cases}
$$

for all $j=1, \ldots, r$. Then the operator $T(w, \mu, \delta, \nu)$ has no zero eigenvalues, for every $K$-type $\mu$ containing $\delta$.

Proof. Decompose $T(w, \mu, \delta, \nu)$ in factors corresponding to simple reflections, as in equation (6.4). Because the map

$$
F \mapsto \mu_{\delta}\left(\sigma_{\alpha_{j}}\right)\left(F \circ \mu\left(\sigma_{\alpha_{j}}^{-1}\right)\right)
$$


is invertible, the operator $T\left(s_{\alpha_{j}}, \mu, \delta_{j-1}, \nu_{j-1}\right)$ is invertible if and only if

$$
d_{k}\left(\alpha_{j}, \nu_{j-1}\right) \neq 0
$$

for all $k \in \mathbb{N} / 2$ such that the subspace $\operatorname{Hom}_{M}\left(V_{\mu}^{\alpha_{j}}( \pm k), \delta_{j-1}\right) \neq\{0\}$. This condition is certainly met if we require that:

$$
\begin{cases}d_{k}\left(\alpha_{j}, \nu_{j-1}\right) \neq 0, \forall k \in 2 \mathbb{N}+2 & \text { if } \alpha_{j} \text { is non-metaplectic and good for } \delta_{j-1}, \\ d_{k}\left(\alpha_{j}, \nu_{j-1}\right) \neq 0, \forall k \in 2 \mathbb{N}+3 & \text { if } \alpha_{j} \text { is non-metaplectic and bad for } \delta_{j-1}, \\ d_{k}\left(\alpha_{j}, \nu_{j-1}\right) \neq 0, \forall k \in \mathbb{N}+\frac{3}{2} & \text { if } \alpha_{j} \text { is metaplectic. }\end{cases}
$$

The constants $d_{k}$ are given in equations (6.5) and (6.6). It is clear that the above condition is equivalent to:

$$
\begin{cases}\left\langle\nu_{j-1}, \check{\alpha}_{j}\right\rangle \notin 2 \mathbb{N}+1 & \text { if } \alpha_{j} \text { is non-metaplectic and good for } \delta_{j-1}, \\ \left\langle\nu_{j-1}, \check{\alpha_{j}}\right\rangle \notin 2 \mathbb{N}+2 & \text { if } \alpha_{j} \text { is non-metaplectic and bad for } \delta_{j-1}, \\ \left\langle\nu_{j-1}, \check{\alpha_{j}}\right\rangle \notin \mathbb{N}+\frac{1}{2} & \text { if } \alpha_{j} \text { is metaplectic. }\end{cases}
$$

It is convenient to rephrase (6.11) in terms of $\delta$ and $\nu$. Assume $j>1$. Then

$\left\langle\nu_{j-1}, \check{\alpha_{j}}\right\rangle=\left\langle x_{j-1} \cdot \nu, \check{\alpha_{j}}\right\rangle=\left\langle s_{\alpha_{j-1}} \cdots s_{\alpha_{1}} \cdot \nu, \check{\alpha_{j}}\right\rangle=\left\langle\nu, s_{\alpha_{1}} \cdots s_{\alpha_{j-1}} \cdot \check{\alpha_{j}}\right\rangle=\left\langle\nu, x_{j-1}^{-1} \cdot \check{\alpha_{j}}\right\rangle$.

Moreover, $\alpha_{j}$ is good for $\delta_{j-1}$ if and only if $x_{j-1}^{-1} \cdot \alpha_{j}$ is good for $\delta$ :

$s_{\alpha_{j}} \cdot \delta_{j-1}=\delta_{j-1} \Leftrightarrow s_{\alpha_{j}} \cdot\left(x_{j-1} \cdot \delta\right)=x_{j-1} \cdot \delta \Leftrightarrow\left(x_{j-1}^{-1} s_{\alpha_{j}} x_{j-1}\right) \cdot \delta=\delta \Leftrightarrow s_{x_{j-1}^{-1} \cdot \alpha_{j}} \cdot \delta=\delta$.

These conditions hold trivially for $j=1$, because $x_{0}=I d$. Finally, observe that

$\alpha_{j}$ is metaplectic $\Leftrightarrow \alpha_{j}$ is long $\Leftrightarrow x_{j-1}^{-1} \cdot \alpha_{j}$ is long $\Leftrightarrow x_{j-1}^{-1} \cdot \alpha_{j}$ is metaplectic.

We conclude that the intertwining operator $T(\omega, \mu, \delta, \nu$ is invertible if

$$
\begin{cases}\left\langle\nu, x_{j-1}^{-1} \cdot \check{\alpha_{j}}\right\rangle \notin 2 \mathbb{N}+1 & \text { if } x_{j-1}^{-1} \cdot \alpha_{j} \text { is non-metaplectic and good for } \delta, \\ \left\langle\nu, x_{j-1}^{-1} \cdot \check{\alpha_{j}}\right\rangle \notin 2 \mathbb{N}+2 & \text { if } x_{j-1}^{-1} \cdot \alpha_{j} \text { is non-metaplectic and bad for } \delta, \\ \left\langle\nu, x_{j-1}^{-1} \cdot \check{\alpha_{j}}\right\rangle \notin \mathbb{N}+\frac{1}{2} & \text { if } x_{j-1}^{-1} \cdot \alpha_{j} \text { is metaplectic }\end{cases}
$$

for all $j=1, \ldots, r$. There is a more compact way to state this condition. Recall that every metaplectic root is good for $\delta$ and that

$$
\check{\beta}= \begin{cases}\beta & \text { if } \beta \text { is non-metaplectic (i.e., short), } \\ \frac{1}{2} \beta & \text { if } \beta \text { is metaplectic (i.e., long) }\end{cases}
$$

for all $\beta \in \Delta$. Hence condition (6.12) is equivalent to:

$$
\begin{cases}\left\langle\nu, x_{j-1}^{-1} \cdot \alpha_{j}\right\rangle \notin 2 \mathbb{N}+1 & \text { if } x_{j-1}^{-1} \cdot \alpha_{j} \text { is good for } \delta, \\ \left\langle\nu, x_{j-1}^{-1} \cdot \alpha_{j}\right\rangle \notin 2 \mathbb{N}+2 & \text { if } x_{j-1}^{-1} \cdot \alpha_{j} \text { is bad for } \delta\end{cases}
$$

for all $j=1, \ldots, r$. (Note that this condition is independent of $\mu$.)

Corollary 6.5. Suppose that

$$
\begin{cases}\langle\nu, \beta\rangle \notin 2 \mathbb{Z}+1 & \text { for all roots } \beta \text { that are good for } \delta, \\ \langle\nu, \beta\rangle \notin 2 \mathbb{Z} \backslash\{0\} & \text { for all roots } \beta \text { that are bad for } \delta .\end{cases}
$$

Then the operator $T(w, \mu, \delta, \nu)$ has no zero eigenvalues, for every $K$-type $\mu$ containing $\delta$, and for all $w \in W$. 


\section{Non-Unitarity CERTIFICATES FOR GEnUine LANGLANDS QUOTIENTS}

In this section, we discuss the role of petite $K$-types in the determination of non-unitarity certificates for genuine Langlands quotients of $M p(2 n)$.

7.1. Operators on genuine petite $K$-types. Let $\delta$ be a genuine $M$-type. For every $K$-type $\mu$ containing $\delta$, let $\psi_{\mu}$ be the representation of $W^{\delta}$ on the space $V_{\mu}[\delta]:=\operatorname{Hom}_{M}(\mu, \delta)$ introduced in Section 5.1. If $\mu$ is petite, i.e., is level at most two, the intertwining operator $T\left(w_{0}, \mu, \delta, \nu\right)$ can be computed by means of Weyl group calculations, and depends only on the $W^{\delta}$-representation $\psi_{\mu}$. To make this precise, we need to introduce some notation. Recall that the long Weyl group element $w_{0}$ stabilizes every $M$-type. In particular, $w_{0} \in W^{\delta}$.

Notation. Let

$$
w_{0}=s_{\beta_{r}} \cdots s_{\beta_{2}} s_{\beta_{1}}
$$

be a minimal decomposition of $w_{0}$ in $W^{\delta}=W\left(\Delta_{\delta}\right)$. For each $W^{\delta}$-representation $\psi$ on a space $V_{\psi}$, and each (real) character $\nu$ of $A$, define the following operators on $V_{\psi}:$

$$
\check{A}\left(w_{0}, \psi, \nu\right):=\prod_{j=1}^{r} \check{A}\left(s_{\beta_{j}}, \psi, \nu_{j-1}\right)
$$

with $\nu_{0}=\nu, \nu_{j}=s_{\beta_{j}} s_{\beta_{j-1}} \cdots s_{\beta_{1}} \cdot \nu$ for $j>1$, and

$$
\check{A}\left(s_{\beta_{j}}, \psi, \nu_{j-1}\right):=\frac{I d+\left\langle\beta_{j}, \nu_{j-1}\right\rangle \psi\left(s_{\beta_{j}}\right)}{1+\left\langle\beta_{j}, \nu_{j-1}\right\rangle} .
$$

We call $\check{A}\left(s_{\beta_{j}}, \psi, \nu_{j-1}\right)$ the $\check{\beta}_{j}$-factor of $\check{A}\left(w_{0}, \psi_{\mu}, \nu\right)$.

Theorem 7.1. Let $\mu$ be a genuine $K$-type containing $\delta$, and let $\psi_{\mu}$ be the representation of $W^{\delta}$ on the space $V_{\mu}[\delta]:=\operatorname{Hom}_{M}(\mu, \delta)$. If $\mu$ is petite, the intertwining operator $T\left(w_{0}, \mu, \delta, \nu\right)$ depends only on the $W^{\delta}$-structure of $V_{\mu}[\delta]$. Precisely:

$$
T\left(w_{0}, \mu, \delta, \nu\right)=\check{A}\left(w_{0}, \psi_{\mu}, \nu\right),
$$

for all $\nu \in \mathfrak{a}_{\mathbb{R}}^{*}$.

Proof. Both operators in equation (7.4) can be decomposed as a product of operators corresponding to simple reflections. The factorization of $T\left(w_{0}, \mu, \delta, \nu\right)$ mimics a minimal factorization of the long Weyl group element $w_{0}$ in $W$, the one of $\check{A}\left(w_{0}, \psi_{\mu}, \nu\right)$ mimics a minimal factorization of $w_{0}$ in $W^{\delta}$. We need to choose these two factorizations of $w_{0}$ in a "compatible" fashion. Assume that

$$
w_{0}=s_{\beta_{r}} \cdots s_{\beta_{2}} s_{\beta_{1}}
$$

is a minimal decomposition of $w_{0}$ in $W^{\delta}$. For each root $\beta$ which is simple in $W^{\delta}$, but not simple in $W$, we choose a minimal decomposition of $s_{\beta}$ in $W$ of the form:

$$
s_{\beta}=\left(s_{\eta_{1}} s_{\eta_{2}} \cdots s_{\eta_{l}}\right) s_{\xi}\left(s_{\eta_{l}} \cdots s_{\eta_{2}} s_{\eta_{1}}\right)
$$

with

- $\xi \operatorname{good}$ for $\left(s_{\eta_{l}} \cdots s_{\eta_{2}} s_{\eta_{1}}\right) \delta$, and

- $\eta_{j}$ bad for both $\left(s_{\eta_{j-1}} \cdots s_{\eta_{2}} s_{\eta_{1}}\right) \delta$ and $\left(s_{\eta_{j+1}} \cdots s_{\eta_{l}} s_{\xi} s_{\eta_{l}} \cdots s_{\eta_{1}}\right) \delta$. 
(Such a decomposition always exists, as the following example illustrates.) We require that, after replacing every reflection $s_{\beta}$ in (7.1) which is not simple in $W$ by its expression in (7.6), we obtain a minimal decomposition

$w_{0}=s_{\beta_{r}} \cdots(\underbrace{s_{\zeta_{1}} s_{\varsigma_{2}} \cdots s_{\varsigma_{n}} s_{\zeta} s_{\varsigma_{n}} \cdots s_{\varsigma_{2}} s_{\varsigma_{1}}}_{s_{\beta_{j}}}) s_{\beta_{i-1}} \cdots(\underbrace{s_{\eta_{1}} s_{\eta_{2}} \cdots s_{\eta_{l}} s_{\xi} s_{\eta_{l}} \cdots s_{\eta_{2}} s_{\eta_{1}}}_{s_{\beta_{i}}}) \cdots s_{\beta_{1}}$

of $w_{0}$ in $W$. We will prove that:

(a) If $\beta$ is a good root for $\delta$, and $\beta$ is simple in both $W^{\delta}$ and $W$, then the $\breve{\beta}$ factor of $\check{A}\left(w_{0}, \psi_{\mu}, \nu\right)$ matches the corresponding $\beta$-factor of $T\left(w_{0}, \mu, \delta, \nu\right)$.

(b) If $\beta$ is a good root for $\delta$, and $\beta$ is simple in $W^{\delta}$ but not in $W$, then the $\widetilde{\beta}$ factor of $\check{A}\left(w_{0}, \psi_{\mu}, \nu\right)$ matches the product of all the factors of $T\left(w_{0}, \mu, \delta, \nu\right)$ coming from the minimal decomposition (7.6) of $s_{\beta}$ in $W$.

Here is an example. Refer to Section 3.2 for the notation. Let $G$ be $M p(10)$, and let $\delta$ be the genuine $M$-type $\delta_{2,3}$. Note that $W$ and $W^{\delta}$ are Weyl groups of type $C_{5}$ and $C_{2} \times C_{3}$, respectively. We choose

$$
\left\{\epsilon_{i} \pm \epsilon_{j} \mid 1 \leq i<j \leq 5\right\} \cup\left\{2 \epsilon_{k} \mid k=1, \ldots, 5\right\}
$$

to be the positive roots in $\Delta$ (with $\epsilon_{1}-\epsilon_{2}, \epsilon_{2}-\epsilon_{3}, \epsilon_{3}-\epsilon_{4}, \epsilon_{4}-\epsilon_{5}$ and $2 \epsilon_{5}$ simple), and

$$
\left\{\epsilon_{1} \pm \epsilon_{2}, 2 \epsilon_{1}, 2 \epsilon_{2}\right\} \cup\left\{\epsilon_{3} \pm \epsilon_{4}, \epsilon_{3} \pm \epsilon_{5}, \epsilon_{4} \pm \epsilon_{5}, 2 \epsilon_{3}, 2 \epsilon_{4}, 2 \epsilon_{5}\right\}
$$

to be the positive roots in $\Delta_{\delta}$ (with $\epsilon_{1}-\epsilon_{2}, 2 \epsilon_{2}, \epsilon_{3}-\epsilon_{4}, \epsilon_{4}-\epsilon_{5}$ and $2 \epsilon_{5}$ simple). The long Weyl group element $w_{0}=-I d$ has length 13 in $W^{\delta}$ and length 25 in $W$. In $W^{\delta}$, we decompose it as:

$$
w_{0}=\left(s_{\epsilon_{1}-\epsilon_{2}} s_{2 \epsilon_{2}} s_{\epsilon_{1}-\epsilon_{2}}\right) s_{2 \epsilon_{2}}\left(s_{\epsilon_{3}-\epsilon_{4}} s_{\epsilon_{4}-\epsilon_{5}} s_{2 \epsilon_{5}} s_{\epsilon_{4}-\epsilon_{5}} s_{\epsilon_{3}-\epsilon_{4}}\right)\left(s_{\epsilon_{4}-\epsilon_{5}} s_{2 \epsilon_{5}} s_{\epsilon_{4}-\epsilon_{5}}\right) s_{2 \epsilon_{5}} .
$$

Note that $2 \epsilon_{2}$ is the only good root which is simple in $\Delta_{\delta}$ but not in $\Delta$. We claim that

$$
s_{2 \epsilon_{2}}=s_{\epsilon_{2}-\epsilon_{3}} s_{\epsilon_{3}-\epsilon_{4}} s_{\epsilon_{4}-\epsilon_{5}} s_{2 \epsilon_{5}} s_{\epsilon_{4}-\epsilon_{5}} s_{\epsilon_{3}-\epsilon_{4}} s_{\epsilon_{2}-\epsilon_{3}}
$$

is a minimal decomposition of $s_{2 \epsilon_{2}}$ in $W$ that satisfies all the required properties.

For all $\gamma \in \mathfrak{a}_{\mathbb{R}}^{*}$, the matching of operators is as follows:

- If $\beta=2 \epsilon_{5}, \epsilon_{1}-\epsilon_{2}, \epsilon_{3}-\epsilon_{4}$ or $\beta=\epsilon_{4}-\epsilon_{5}$, then

$$
\check{A}\left(s_{\beta}, \psi_{\mu}, \gamma\right)=T\left(s_{\beta}, \mu, \delta, \gamma\right) \text {. }
$$

- If $\beta=2 \epsilon_{2}$, then

$$
\begin{aligned}
\check{A}\left(s_{2 \epsilon_{2}}, \psi_{\mu}, \gamma\right)=T\left(s_{\epsilon_{2}-\epsilon_{3}}, \mu, \delta_{1}, s_{\epsilon_{3}-\epsilon_{4}} s_{\epsilon_{4}-\epsilon_{5}} s_{2 \epsilon_{2}} s_{\epsilon_{4}-\epsilon_{5}} s_{\epsilon_{3}-\epsilon_{4}} s_{\epsilon_{2}-\epsilon_{3}} \gamma\right) \\
\circ T\left(s_{\epsilon_{3}-\epsilon_{4}}, \mu, \delta_{2}, s_{\epsilon_{4}-\epsilon_{5}} s_{2 \epsilon_{2}} s_{\epsilon_{4}-\epsilon_{5}} s_{\epsilon_{3}-\epsilon_{4}} s_{\epsilon_{2}-\epsilon_{3}} \gamma\right) \\
\circ T\left(s_{\epsilon_{4}-\epsilon_{5}}, \mu, \delta_{3}, s_{2 \epsilon_{2}} s_{\epsilon_{4}-\epsilon_{5}} s_{\epsilon_{3}-\epsilon_{4}} s_{\epsilon_{2}-\epsilon_{3}} \gamma\right) \\
\circ T\left(s_{2 \epsilon_{2}}, \mu, \delta_{3}, s_{\epsilon_{4}-\epsilon_{5}} s_{\epsilon_{3}-\epsilon_{4}} s_{\epsilon_{2}-\epsilon_{3}} \gamma\right) \\
\circ T\left(s_{\epsilon_{4}-\epsilon_{5}}, \mu, \delta_{2}, s_{\epsilon_{3}-\epsilon_{4}} s_{\epsilon_{2}-\epsilon_{3}} \gamma\right) \\
\circ T\left(s_{\epsilon_{3}-\epsilon_{4}}, \mu, \delta_{1}, s_{\epsilon_{2}-\epsilon_{3}} \gamma\right) \circ T\left(s_{\epsilon_{2}-\epsilon_{3}}, \mu, \delta, \gamma\right)
\end{aligned}
$$


We now give the proof of the theorem.

For part $(a)$, we assume that the root $\beta$ is good for $\delta$. Recall from Section 5.3 that for every genuine petite $K$-type $\mu$, the space $V_{\mu}[\delta]$ decomposes as

$$
V_{\mu}[\delta]=\underbrace{\operatorname{Hom}_{M}\left(V_{\mu}^{\beta}( \pm 1 / 2), \delta\right)}_{(+1) \text {-eigenspace of } \psi_{\mu}\left(s_{\beta}\right)} \oplus \underbrace{\operatorname{Hom}_{M}\left(V_{\mu}^{\beta}( \pm 3 / 2), \delta\right)}_{(-1) \text {-eigenspace of } \psi_{\mu}\left(s_{\beta}\right)}
$$

if $\beta$ is metaplectic, and

$$
V_{\mu}[\delta]=\underbrace{\operatorname{Hom}_{M}\left(V_{\mu}^{\beta}(0), \delta\right)}_{(+1) \text {-eigenspace of } \psi_{\mu}\left(s_{\beta}\right)} \oplus \underbrace{\operatorname{Hom}_{M}\left(V_{\mu}^{\beta}( \pm 2), \delta\right)}_{(-1) \text {-eigenspace of } \psi_{\mu}\left(s_{\beta}\right)}
$$

if $\beta$ is non-metaplectic. Because $\beta$ is good for $\delta$, the operator $T\left(s_{\beta}, \mu, \delta, \gamma\right)$ acts on each subspace $\operatorname{Hom}_{M}\left(V_{\mu}^{\beta}( \pm k), \delta\right)$ by the scalar $b_{k}(\beta, \gamma)$ (see Proposition 6.2). Recall that

$$
b_{k}(\beta, \gamma)= \begin{cases}1 & \text { if } k=0 \text { or } k=1 / 2 \\ \frac{1 / 2-\langle\gamma, \check{\beta}\rangle}{1 / 2+\langle\gamma, \check{\beta}\rangle} & \text { if } k=3 / 2 \\ \frac{1-\langle\gamma, \breve{\beta}\rangle}{1+\langle\gamma, \ddot{\beta}\rangle} & \text { if } k=2\end{cases}
$$

and

$$
\check{\beta}= \begin{cases}\beta & \text { if } \beta \text { is non-metaplectic, } \\ \frac{1}{2} \beta & \text { if } \beta \text { is metaplectic. }\end{cases}
$$

Hence, we can rewrite these constants as:

$$
b_{k}(\beta, \gamma)= \begin{cases}1 & \text { if } k=0, \frac{1}{2} \text { or } 1 \\ \frac{1-\langle\gamma, \beta\rangle}{1+\langle\gamma, \beta\rangle} & \text { if } k=\frac{3}{2} \text { or } 2\end{cases}
$$

It follows that for every good root $\beta$ (metaplectic or not), the operator $T\left(s_{\beta}, \mu, \delta, \gamma\right)$ acts on $V_{\mu}[\delta]$ by

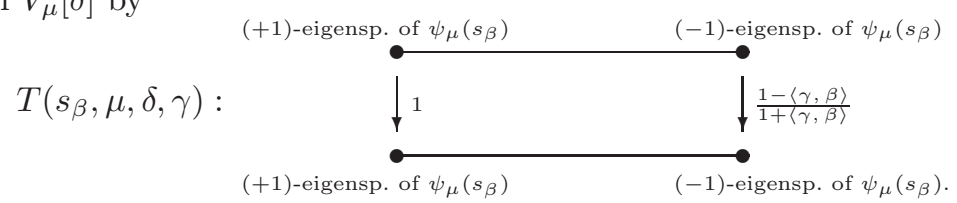

This is exactly the action of the $\check{\beta}$-factor $\check{A}\left(s_{\beta}, \psi_{\mu}, \gamma\right)$.

For part $(b)$, we assume that the $\operatorname{root} \beta$ is simple in $W^{\delta}$ but not in $W$, and we prove that $\check{A}\left(s_{\beta}, \psi_{\mu}, \gamma\right)$ matches the product of all the factors of $T\left(w_{0}, \mu, \delta, \nu\right)$ coming from the minimal decomposition

$$
s_{\beta}=\left(s_{\eta_{1}} s_{\eta_{2}} \cdots s_{\eta_{l}}\right) s_{\xi}\left(s_{\eta_{l}} \cdots s_{\eta_{2}} s_{\eta_{1}}\right)
$$

of $s_{\beta}$ in $W$. For brevity of notation, set:

$$
\begin{aligned}
\delta_{j}:=s_{\eta_{j}} \ldots s_{\eta_{1}} \delta & \forall j=1, \ldots, l, \\
\gamma_{j}:=s_{\eta_{j}} \ldots s_{\eta_{1}} \gamma & \forall j=1, \ldots, l, \\
\gamma_{l}^{\prime}:=s_{\xi} s_{\eta_{l}} \ldots s_{\eta_{1}} \gamma & \text { and } \\
\gamma_{j}^{\prime}:=s_{\eta_{j+1}} \ldots s_{\eta_{l}} s_{\xi} s_{\eta_{l}} \ldots s_{\eta_{1}} \gamma & \forall j=1, \ldots, l-1 .
\end{aligned}
$$

Then the claim is that

$$
\check{A}\left(s_{\beta}, \psi_{\mu}, \gamma\right)=T\left(s_{\eta_{1}}, \mu, \delta_{1}, \gamma_{1}^{\prime}\right) \circ T\left(s_{\eta_{2}}, \mu, \delta_{2}, \gamma_{2}^{\prime}\right) \circ \cdots \circ T\left(s_{\eta_{l}}, \mu, \delta_{l}, \gamma_{l}^{\prime}\right)
$$

$$
\circ T\left(s_{\xi}, \mu, \delta_{l}, \gamma_{l}\right) \circ T\left(s_{\eta_{l}}, \mu, \delta_{l-1}, \gamma_{l-1}\right) \circ \cdots \circ T\left(s_{\eta_{2}}, \mu, \delta_{1}, \gamma_{1}\right) \circ T\left(s_{\eta_{1}}, \mu, \delta, \gamma\right) \text {. }
$$


On the left, we have the operator

$$
\check{A}\left(s_{\beta}, \psi_{\mu}, \gamma\right):=\frac{I d+\langle\gamma, \beta\rangle \psi_{\mu}\left(s_{\beta}\right)}{1+\langle\gamma, \beta\rangle}
$$

of $V_{\mu}[\delta]$. On the right, we have a composition of operators. We point out that although the product acts on $V_{\mu}[\delta]$, the single factors do not:

$$
\begin{aligned}
T\left(s_{\eta_{j}}, \mu, \delta_{j-1}, \gamma_{j-1}\right): V_{\mu}\left[\delta_{j-1}\right] \rightarrow V_{\mu}\left[\delta_{j}\right] & j=1, \ldots, l, \\
T\left(s_{\xi}, \mu, \delta_{l}, \gamma_{l}\right): V_{\mu}\left[\delta_{l}\right] \rightarrow V_{\mu}\left[\delta_{l}\right], & \\
T\left(s_{\eta_{j}}, \mu, \delta_{j}, \gamma_{j}^{\prime}\right): V_{\mu}\left[\delta_{j}\right] \rightarrow V_{\mu}\left[\delta_{j-1}\right] & j=1, \ldots, l .
\end{aligned}
$$

(Here $\delta_{0}=\delta$ and $\gamma_{0}=\gamma$.) Let us look more closely at these factors. The root $\eta_{j}$ is bad for both $\delta_{j-1}$ and $\delta_{j}$ and is necessarily non-metaplectic (because metaplectic roots are good for every genuine $M$-type). By our hypothesis, $\mu$ is level two, so the only possible eigenvalue of $\left(\mathrm{d} \mu\left(Z_{\eta_{j}}\right)\right)^{2}$ on the isotypic components of both $\delta_{j-1}$ and $\delta_{j}$ inside $\mu$ is $(-1)$. It follows that the operators $T\left(s_{\eta_{j}}, \mu, \delta_{j-1}, \gamma_{j-1}\right)$ and $T\left(s_{\eta_{j}}, \mu, \delta_{j}, \gamma_{j}^{\prime}\right)$ act by

$$
T \mapsto 1 \cdot \mu_{\delta}\left(\sigma_{\eta_{j}}\right) T \mu\left(\sigma_{\eta_{j}}\right)^{-1} .
$$

This holds true for all $j=1, \ldots, l$. We are left with the central factor, $T\left(s_{\xi}, \mu, \delta_{l}, \gamma_{l}\right)$. The root $\xi$ is good for $\delta_{l}$; hence (by essentially the same argument used in part $a$ ) the operator $T\left(s_{\xi}, \mu, \delta_{l}, \gamma_{l}\right)$ acts on $V_{\mu}\left[\delta_{l}\right]$ by

$$
T \mapsto \frac{T+\left\langle\gamma_{l}, \xi\right\rangle \mu_{\delta}\left(\sigma_{\xi}\right) T \mu\left(\sigma_{\xi}\right)^{-1}}{1+\left\langle\gamma_{l}, \xi\right\rangle} .
$$

Composing all these operators, we obtain the map:

$$
T \mapsto \frac{T+\left\langle\gamma_{l}, \xi\right\rangle \mu_{\delta}\left(\sigma_{\beta}\right) T \mu\left(\sigma_{\beta}\right)^{-1}}{1+\left\langle\gamma_{l}, \xi\right\rangle} .
$$

Note that

$$
\left\langle\gamma_{l}, \xi\right\rangle=\left\langle s_{\eta_{l}} \ldots s_{\eta_{1}} \gamma, \xi\right\rangle=\left\langle\gamma, s_{\eta_{1}} \ldots s_{\eta_{l}} \xi\right\rangle=\langle\gamma, \beta\rangle
$$

and that

$$
\mu_{\delta}\left(\sigma_{\beta}\right) T \mu\left(\sigma_{\beta}\right)^{-1}=\psi_{\mu}\left(s_{\beta}\right) T
$$

(because $\beta$ is a good root for $\delta$ ). Hence we can rewrite (7.15) as

$$
T \mapsto \frac{T+\langle\gamma, \beta\rangle \psi_{\mu}\left(s_{\beta}\right) T}{1+\langle\gamma, \beta\rangle} .
$$

This shows that the composition of operators in equation (7.11) matches the operator $\check{A}\left(s_{\beta}, \psi_{\mu}, \gamma\right)$ and concludes the proof of the theorem.

Notation. Let $\Delta_{\delta}$ be the system of good roots for $\delta$. Define $G^{\delta}$ to be the (classical) real connected split group corresponding to the root system which is dual to $\Delta_{\delta}$.

E. $g$., if $\delta=\delta_{p, q}$ and $\Delta_{\delta}=C_{p} \times C_{q}$, define $G^{\delta} \simeq S O(p+1, p)_{0} \times S O(q+1, q)_{0}$.

Proposition 7.2. If the $W^{\delta}$-type $\psi_{\mu}$ is relevant, then there is a petite quasispherical $K$-type $\tau_{\psi_{\mu}}$ of $G^{\delta}$ such that

$$
T^{M p(2 n)}\left(w_{0}, \mu, \delta, \nu\right)=T^{G^{\delta}}\left(w_{0}, \tau_{\psi_{\mu}}, \delta_{0}, \nu\right) .
$$

The operator on the left is a genuine intertwining operator for $M p(2 n)$; the one on the right is a spherical intertwining operator for $G^{\delta}$. 
Proof. This follows from Theorems 2.5. 2.9 and 7.1 and from the fact that, for each $\beta$, the $\breve{\beta}$-factor of the operator $\check{A}\left(w_{0}, \nu, \delta\right)$ for $M p(2 n)$ matches the $\beta$-factor of the operator $A\left(w_{0}, \nu, \delta\right)$ for $G^{\delta}$ (compare equations (7.3) and (2.11)).

7.2. Non-unitarity certificates for genuine Langlands quotients. Because

$$
J(\delta, \nu) \simeq J(w \delta, w \nu) \quad \forall w \in W
$$

it suffices to consider a single $M$-type in each $W$-orbit for the action of the Weyl group on $\widehat{M}$. (If $\tau=w \cdot \delta$, then $C S(G, \tau)=w^{-1} \cdot C S(G, \delta)$.)

Orbits of $M$-types are parameterized by pairs of non-negative integers $(p, q)$ such that $p+q=n$. In each orbit, we choose the representative $\delta_{p, q}$ introduced in Section 5.1. This is the unique genuine irreducible representation of $M$ satisfying

$$
\delta\left(m_{2 \epsilon_{k}}\right)= \begin{cases}+i & \text { if } k=1, \ldots, p, \\ -i & \text { if } k=p+1, \ldots, n .\end{cases}
$$

The corresponding fine $K$-type is:

$$
\mu_{\delta_{p, q}}:=(\underbrace{\frac{1}{2}, \ldots, \frac{1}{2}}_{p}, \underbrace{-\frac{1}{2}, \ldots,-\frac{1}{2}}_{q}) .
$$

For all $\nu \in \mathfrak{a}_{\mathbb{R}}^{*}$, let $J\left(\delta_{p, q}, \nu\right)$ be the Langlands quotient with parameter $\nu$, that is, the distinguished composition factor of the principal series $I\left(\delta_{p, q}, \nu\right)$ containing $\mu_{\delta_{p, q}}$. We know that $J\left(\delta_{p, q}, \nu\right)$ is Hermitian for all values of $\nu$ and is unitary if and only if $\nu$ satisfies the condition:

$$
T\left(w_{0}, \mu, \delta_{p, q}, \nu\right) \text { is positive semi-definite, for all } \mu \in \widehat{K} \text {. }
$$

Definition 7.3 (cf. [3]). Let $\Sigma \subset \widehat{K}$ be a set of $K$-types. We say that $J\left(\delta_{p, q}, \nu\right)$ is unitary on $\Sigma$ if its invariant form is positive semi-definite on the $\mu$-isotypic component of $J\left(\delta_{p, q}, \nu\right)$ for all $\mu \in \Sigma$. Equivalently, $J\left(\delta_{p, q}, \nu\right)$ is unitary on $\Sigma$ if and only if the operator $T\left(w_{0}, \mu, \delta_{p, q}, \nu\right)$ is positive semi-definite for all $\mu \in \Sigma$.

Note that $J\left(\delta_{p, q}, \nu\right)$ is unitary if and only if it is unitary on $\widehat{K}$. Because $\widehat{K}$ is an infinite set, this condition is hard to check. We will restrict our attention to the (finite) set $\Sigma_{K}\left(G, \delta_{p, q}\right)$ of petite $K$-types containing $\delta_{p, q}$.

By Theorem [7.1, the intertwining operator on a petite $K$-type matches a spherical operator for the $p$-adic split group $G^{\delta_{p, q}}(\mathbb{F})$ whose root system is dual to the set $\Delta_{\delta_{p, q}}$ of good roots for $\delta_{p, q}$. Because $\Delta_{\delta_{p, q}}$ is a root system of type $C_{p} \times C_{q}$ (see Section [3.4), the corresponding real split group is

$$
G^{\delta_{p, q}}(\mathbb{R}):=S O(p+1, p)_{0} \times S O(q+1, q)_{0} .
$$

Suppose that the Langlands quotient $J\left(\delta_{p, q}, \nu\right)$ of $G$ is unitary. Then $J\left(\delta_{p, q}, \nu\right)$ is unitary on $\Sigma_{K}\left(G, \delta_{p, q}\right)$. By Theorem 7.1, the Langlands quotient $J_{\mathbb{F}}(\nu)$ of $G^{\delta_{p, q}}(\mathbb{F})$

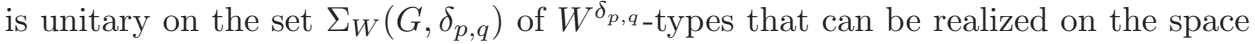
$V_{\mu}\left[\delta_{p, q}\right]$ for some $\mu \in \Sigma_{K}\left(G, \delta_{p, q}\right)$. (The notation is as in Section 5.1]) By Proposi-

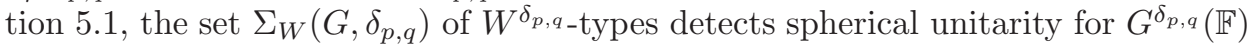
(see Definition 2.77) hence the spherical quotient $J_{\mathbb{F}}(\nu)$ is unitary. By Theorem 2.10. the spherical module for the real split group $G^{\delta_{p, q}}(\mathbb{R})$ is also unitary. The result is an embedding of unitary duals: the $\delta_{p, q}$-complementary series of $G$ is embedded into the spherical unitary dual of $S O(p+1, p)_{0} \times S O(q+1, q)_{0}$. 
Notation. Let $C S\left(S O(p+1, p)_{0}, \delta_{0}\right)$ and $C S\left(S O(q+1, q)_{0}, \delta_{0}\right)$ denote the spherical complementary series of $S O(p+1, p)_{0}$ and $S O(q+1, q)_{0}$, respectively.

Theorem 7.4. Let $G$ be $M p(2 n)$ and let $\nu=\left(\nu_{1}, \ldots, \nu_{n}\right)$ be a real character of $A$. For each pair of non-negative integers $(p, q)$ such that $p+q=n$, let $\delta_{p, q}$ be the genuine representation of $M$ introduced in Section [5.1. Set

$$
\nu^{p}:=\left(\nu_{1}, \ldots, \nu_{p}\right) \quad \nu^{q}:=\left(\nu_{p+1}, \ldots, \nu_{n}\right)
$$

and write $\nu=\left(\nu^{p} \mid \nu^{q}\right)$. The map

$$
\begin{aligned}
C S\left(M p(2 n), \delta_{p, q}\right) & \rightarrow C S\left(S O(p+1, p)_{0}, \delta_{0}\right) \times C S\left(S O(q+1, q)_{0}, \delta_{0}\right) \\
\nu & \mapsto\left(\nu^{p}, \nu^{q}\right)
\end{aligned}
$$

is a well-defined injection.

Corollary 7.5. Let $G$ be $M p(2 n)$ and let $\nu=\left(\nu_{1}, \ldots, \nu_{n}\right)=\left(\nu^{p} \mid \nu^{q}\right)$ be a real character of $A$. If the spherical Langlands quotient $J\left(\delta_{0}, \nu^{p}\right)$ of $S O(p+1, p)_{0}$ and/or the spherical Langlands quotient $J\left(\delta_{0}, \nu^{q}\right)$ of $S O(q+1, q)_{0}$ are not unitary, then the genuine Langlands quotient $J\left(\delta_{p, q}, \nu\right)$ of $M p(2 n)$ is also not unitary.

Remark 7.6. The same result holds if we replace the identity component of each special orthogonal group by the corresponding full special orthogonal group, or even by the full orthogonal group, because the spherical complementary series are the same.

The unitary spherical spectrum of split groups of type $B$ is known, by work of D. Barbasch (cf. 4). Hence Theorem 7.4 and Corollary 7.5 provide a set of non-unitarity certificates for genuine irreducible representations of $M p(2 n)$. The following proposition shows the strength of these certificates.

Proposition 7.7. Let $G$ be $M p(2 n)$ and let $\nu=\left(\nu_{1}, \ldots, \nu_{n}\right)$ be a real character of $A$. The complementary series $C S\left(M p(2 n), \delta_{p, q}\right)$ is invariant under conjugation by the stabilizer of $\delta_{p, q}$ (which is of the form $W\left(C_{p}\right) \times W\left(C_{q}\right)$ ), so we may assume that

$$
\nu_{1} \geq \cdots \geq \nu_{p} \geq 0 \quad \text { and } \quad \nu_{p+1} \geq \cdots \geq \nu_{n} \geq 0 .
$$

Suppose that any of the following conditions holds:

(1) $\nu_{p}>1 / 2$

(2) $\nu_{n}>1 / 2$,

(3) $\nu_{a}-\nu_{a+1}>1$, for some a with $1 \leq a \leq p-1$, or

(4) $\nu_{a}-\nu_{a+1}>1$, for some a with $p+1 \leq a \leq n-1$.

Then the genuine Langlands quotient $J\left(\delta_{p, q}, \nu\right)$ of $M p(2 n)$ is not unitary.

Proof. This is an immediate consequence of the analogous non-unitarity certificates for spherical Langlands quotients of $O(p+1, p)$ and $O(q+1, q)$ (cf. [3], Lemma 14.6).

We conclude by stating our main conjecture. (The notation is as in Theorem 7.4.) 
Conjecture 7.8. Let $G$ be $M p(2 n)$. For each pair of non-negative integers $(p, q)$ such that $p+q=n$, the map

$$
\begin{aligned}
C S\left(M p(2 n), \delta_{p, q}\right) & \rightarrow C S\left(S O(p+1, p)_{0}, \delta_{0}\right) \times C S\left(S O(q+1, q)_{0}, \delta_{0}\right) \\
\nu & \mapsto\left(\nu^{p}, \nu^{q}\right)
\end{aligned}
$$

is a bijection.

Some evidence for this conjecture is given in the next section.

\section{SOME EVIDENCE FOR CONJECTURE 7.8}

In light of Theorem 7.4, proving Conjecture 7.8 is equivalent to the following.

Conjecture 8.1. Let $G$ be $M p(2 n)$. For each pair of non-negative integers $(p, q)$ such that $p+q=n$, let

$$
\nu^{p} \in C S\left(S O(p+1, p)_{0}, \delta_{0}\right) \quad \text { and } \quad \nu^{q} \in C S\left(S O(q+1, q)_{0}, \delta_{0}\right)
$$

be spherical unitary parameters for $S O(p+1, p)_{0}$ and $S O(q+1, q)_{0}$, respectively.

Then the Langlands quotient $J\left(\delta_{p, q},\left(\nu^{p} \mid \nu^{q}\right)\right)$ of $M p(2 n)$ is unitary.

(The notation is as in Theorem 7.4])

Remark 8.2. We may assume $p \geq q$, because

$$
J\left(\delta_{p, q},\left(\nu^{p} \mid \nu^{q}\right)\right) \simeq\left[J\left(\delta_{q, p},\left(\nu^{q} \mid \nu^{p}\right)\right)\right]^{*}
$$

and duality preserves unitarity.

In this section we give some evidence for Conjecture 8.1, including the pseudospherical case, two families of special (non-pseudospherical) examples, as well as some small rank cases.

Some of the arguments use Howe's dual pair correspondence for pairs of the form $(O(p, q), S p(2 n, \mathbb{R}))$, with $p+q$ odd. We start by reviewing some of the results on dual pair correspondence; the basic references are 29, 16] and 2]. See also Section 14 of $[3]$.

8.1. The dual pairs $(O(p, q), S p(2 n, \mathbb{R}))$. Let $\left(G, G^{\prime}\right)$ be a reductive dual pair in $S p(2 N, \mathbb{R})$; i.e., let $G$ and $G^{\prime}$ be reductive subgroups of $S p(2 N, \mathbb{R})$ which are mutual centralizers. The preimages $\widetilde{G}$ and $\widetilde{G}^{\prime}$ of $G$ and $G^{\prime}$, respectively, under the covering map $M p(2 N) \rightarrow S p(2 N, \mathbb{R})$ form a dual pair inside the metaplectic group. If $\omega$ is one of the oscillator representations of $M p(2 N)$, then the restriction of $\omega$ to $\widetilde{G} \cdot \widetilde{G}^{\prime}$ gives rise to a correspondence between irreducible representations of $\widetilde{G}$ and irreducible representations of $\widetilde{G}^{\prime}$, by defining

$$
\pi \leftrightarrow \pi^{\prime} \Longleftrightarrow \operatorname{Hom}_{\tilde{G} \times \widetilde{G}^{\prime}}\left(\omega, \pi \otimes \pi^{\prime}\right) \neq 0 .
$$

All modules and maps are assumed to be in the category of $(\mathfrak{g}, K)$-modules. The correspondence satisfies the following properties.

Theorem 8.3 (Howe). Retain the notation described above.

(1) Equation (8.3) defines a bijection between subsets of the sets of genuine irreducible representations of the groups $\widetilde{G}$ and $\widetilde{G}^{\prime}$.

(2) The correspondence in (8.3) gives rise to a well-defined correspondence of infinitesimal characters. 
We will restrict our attention to dual pairs of the form $(O(p, q), S p(2 n, \mathbb{R}))$ with $p+q$ odd. In this case, the double cover of $S p(2 n, \mathbb{R})$ is metaplectic; the double cover of the orthogonal group is linear. Recall that $\widetilde{O}(p, q)$ has a genuine character $\xi$ such that the map $\pi \leftrightarrow \pi \otimes \xi$ gives a bijection between irreducible representations of $O(p, q)$ and genuine irreducible representations of $\widetilde{O}(p, q)$. Consequently, we can regard the theta correspondence for this dual pair as a correspondence between irreducible representations of $O(p, q)$ and irreducible genuine representations of $M p(2 n)$.

In general, the theta correspondence does not preserve unitarity. However, we have preservation of unitarity in the stable range, which we define here only for dual paris of the form under consideration.

Definition 8.4. The dual pair $(O(p, q), S p(2 n, \mathbb{R}))$ is said to be in the stable range with $O(p, q)$ the smaller member if $n \geq p+q$. It is in the stable range with $S p(2 n, \mathbb{R})$ the smaller member if $\min \{p, q\} \geq 2 n$.

Theorem 8.5 (cf. [13). Suppose $\left(G, G^{\prime}\right)$ is a dual pair in the stable range with $G$ the smaller member. If $\pi$ is an irreducible genuine unitary representation of $\widetilde{G}$, then $\pi$ occurs in the dual pair correspondence. Moreover, if $\pi$ corresponds to $\pi^{\prime}$ in the dual pair correspondence, then $\pi^{\prime}$ is a unitary representation of $\widetilde{G}^{\prime}$.

We will apply this theorem to dual pairs of the form $(O(m+1, m), S p(2 n, \mathbb{R}))$ which are in the stable range with the orthogonal group the smaller member, i.e., to dual pairs $(O(m+1, m), S p(2 n, \mathbb{R}))$ satisfying $n \geq 2 m+1$.

Given a dual pair $\left(G, G^{\prime}\right)$, write $K$ and $K^{\prime}$ for the maximal compact subgroups of $\widetilde{G}$ and $\widetilde{G}^{\prime}$, respectively. Howe associates to each $K$ - and $K^{\prime}$-type a degree and defines a subspace $\mathcal{H}$ of the representation space for $\omega$, called the space of joint harmonics, on which there is a one-to-one correspondence between $K$ - and $K^{\prime}$-types. The following result is a valuable tool in determining the dual pair correspondence explicitly.

Theorem 8.6 (cf. 9]). Suppose that $\pi$ corresponds to $\pi^{\prime}$ in the correspondence for the dual pair $\left(G, G^{\prime}\right)$, and that $\mu$ is a $K$-type of minimal degree occurring in $\pi$. Then $\mu$ occurs in $\mathcal{H}$. Let $\mu^{\prime}$ be the $K^{\prime}$-type corresponding to $\mu$ in $\mathcal{H}$; then $\mu^{\prime}$ occurs in $\pi^{\prime}$ and is of minimal degree.

Recall that the orthogonal group $O(p, q)$ has four one-dimensional characters, which we will denote by triv, det, $\chi_{+-}$, and $\chi_{-+}$. Here $\chi_{+-}$represents the character which restricts to triv $\otimes$ det on $O(p) \times O(q) ; \chi_{-+}$is defined analogously. For dual pairs of the form $(O(m+1, m), S p(2 n, \mathbb{R}))$, the correspondence of $K$-types on the space of joint harmonics is such that

$$
\begin{aligned}
\operatorname{triv} & \leftrightarrow \mu_{\delta_{n, 0}}, \\
\operatorname{triv} \otimes \operatorname{det} & \leftrightarrow \mu_{\delta_{n-m, m}} .
\end{aligned}
$$

The trivial $(O(m+1) \times O(m))$-type occurs for all dual pairs, while triv $\otimes$ det occurs whenever $n \geq m$.

8.2. Pseudospherical unitary representations of $M p(2 n)$. Recall that a representation of $M p(2 n)$ is called pseudospherical if it contains a $\widetilde{U}(n)$-type of the form $\operatorname{det}^{ \pm \frac{1}{2}}$. Adams and Barbasch have determined the theta correspondence explicitly, in terms of Langlands parameters, for all dual pairs of the form $(O(p, q), S p(2 n, \mathbb{R}))$ 
with $p+q=2 n+1$ (cf. [2]). If $q=p-1=n$, they show that spherical representations of $O(n+1, n)$ correspond to pseudospherical representations of $M p(2 n)$ with lowest $\widetilde{U}(n)$-type $\sqrt{\operatorname{det} \text { : }}$

$$
J_{O(n+1, n)}\left(\delta_{0}, \nu\right) \leftrightarrow J_{M p(2 n)}\left(\delta_{n, 0}, \nu\right)
$$

for all choices of $\nu$ allowed. The main result of [3] implies that the correspondence (8.6) preserves unitarity from right to left. (Note that this result also follows from our Theorem [7.4. since $O(n+1, n)$ and $S O(n+1, n)_{0}$ have the same spherical unitary parameters.)

Computations on petite $K$-types show that unitarity is preserved in the reverse direction, as well. This implies the truth of our conjecture in the pseudospherical case.

Theorem 8.7 (cf. [3). The correspondence (8.6) preserves unitarity both ways; hence, it gives a parametrization of the pseudospherical unitary dual of $M p(2 n)$ in terms of the spherical unitary dual of $O(n+1, n)$.

8.3. A family of non-pseudospherical examples. For each non-negative integer $m$, let $\rho_{m}$ be the the $m$-tuple

$$
\rho_{m}=\left(m-\frac{1}{2}, m-\frac{3}{2}, \ldots, \frac{3}{2}, \frac{1}{2}\right),
$$

i.e., the infinitesimal character of the trivial representation of $O(m+1, m)$, and of the oscillator representation of $M p(2 m)$. If $\eta$ is an $n$-tuple of numbers, then we denote by $\left(\rho_{m} \mid \eta\right)$ the $(n+m)$-tuple obtained by adding the entries of $\eta$ to $\rho_{m}$.

Theorem 8.8. The representation $J_{M p(2(p+q))}\left(\delta_{p, q},\left(\rho_{p} \mid \nu\right)\right)$ is unitary, for all $\nu \in$ $C S\left(S O(q+1, q)_{0}, \delta_{0}\right)$ and all $p>q$.

Proof. The main theorem of 2 implies that, in the dual pair correspondence for the pair $(O(q+1, q), S p(2 q, \mathbb{R}))$,

$$
J_{O(q+1, q)}\left(\delta_{0}, \nu\right) \otimes \chi_{+-} \leftrightarrow J_{M p(2 q)}\left(\delta_{0, q}, \nu\right)
$$

for all choices of the parameter $\nu$ allowed. Tensoring by a unitary character and taking duals does not change whether a representation is unitary or not, so this is again a unitarity-preserving bijection. Then, if $\nu$ is in the spherical complementary series of $O(q+1, q)$ (or $\left.S O(q+1, q)_{0}\right)$, the representation $J_{M p(2 q)}\left(\delta_{0, q}, \nu\right)$ of $M p(2 q)$ is unitary. We want to prove that, if $p>q$, the representation $J_{M p(2(p+q))}\left(\delta_{p, q},\left(\rho_{p} \mid \nu\right)\right)$ of $M p(2(p+q))$ is also unitary. Consider the dual pair correspondence for the pair $(O(q+1, q), S p(2(p+q), \mathbb{R}))$; set $\pi=J_{O(q+1, q)}\left(\delta_{0}, \nu\right) \otimes \chi_{+-}$and let $\pi^{\prime}$ be the representation of $M p(2(p+q))$ which corresponds to $\pi$. Because $p>q$, we are in the stable range with the orthogonal group the smallest member; therefore, by Theorem 8.5. $\pi^{\prime}$ is unitary (and non-zero). We will show that $\pi^{\prime}=J_{M p(2(p+q))}\left(\delta_{p, q},\left(\rho_{p} \mid \nu\right)\right)$. The induction principle (cf. Section 8 of [2]) implies that $\pi^{\prime}$ is a constituent of the principal series $I_{M p(2(p+q))}\left(\delta_{p, q},\left(\rho_{p} \mid \nu\right)\right)$. Because triv $\otimes$ det is of minimal degree in $\pi$, the $K$-type triv $\otimes \operatorname{det}$ occurs in $\mathcal{H}$ and, by (8.5), the corresponding $K^{\prime}$-type is $\mu_{\delta_{p, q}}$. By Theorem 8.6. $\mu_{\delta_{p, q}}$ occurs in $\pi^{\prime}$, and is of minimal degree. Hence $\pi^{\prime}$ must be the Langlands quotient $J_{M p(2(p+q))}\left(\delta_{p, q},\left(\rho_{p} \mid \nu\right)\right)$.

8.4. Some small rank examples. In this section, we prove our conjecture for metaplectic groups of rank up to three. Additional small rank examples will be given at the end of Section 8.5 . 
8.4.1. $M p(2)$. For $M p(2)$, we only need to consider the case $p=1, q=0$. It is well known that

$$
C S\left(S O(2,1)_{0}, \delta_{0}\right)=\left[-\frac{1}{2}, \frac{1}{2}\right]
$$

and each of these parameters is unitary for the Langlands quotient $J\left(\delta_{1,0}, \nu\right)$ of $M p(2)$. (Note that this result also follows from Corollary 4.6 or Theorem 8.7.) Hence the conjecture holds.

8.4.2. $M p(4)$. For $M p(4)$, we have to consider two cases:

$$
p=2, q=0 \quad \text { and } \quad p=1, q=1 .
$$

For the pseudospherical case, the claim follows from Theorem 8.7 A picture of the unitary parameters is given in Figure 2. Section 2.

For the case $p=q=1$, note that the product of the two spherical complementary series

$$
C S\left(S O(2,1)_{0}, \delta_{0}\right) \times C S\left(S O(2,1)_{0}, \delta_{0}\right)=\left[-\frac{1}{2}, \frac{1}{2}\right] \times\left[-\frac{1}{2}, \frac{1}{2}\right]
$$

is just the unit square, so the claim follows from Corollary 4.6.

8.4.3. $M p(6)$. For $M p(6)$, we have to consider two cases:

$$
p=3, q=0 \quad \text { and } \quad p=2, q=1 .
$$

For the pseudospherical case, the claim follows from Theorem 8.7 The unitary parameters in the fundamental Weyl chamber $(\mathrm{FWC})$ are:

- The intersection of the FWC with the unit cube: $\left\{0 \leq \nu_{3} \leq \nu_{2} \leq \nu_{1} \leq \frac{1}{2}\right\}$.

- The segment from $\left(\frac{1}{2}, \frac{1}{2}, \frac{1}{2}\right)$ to $\left(1, \frac{1}{2}, 0\right):\left\{\left(\frac{1}{2}+t, \frac{1}{2}, \frac{1}{2}-t\right)\right.$, for $\left.0 \leq t \leq \frac{1}{2}\right\}$.

- The segment from $\left(1, \frac{1}{2}, 0\right)$ to $\left(\frac{3}{2}, \frac{1}{2}, \frac{1}{2}\right):\left\{\left(1+t, \frac{1}{2}, t\right)\right.$, for $\left.0 \leq t \leq \frac{1}{2}\right\}$.

- The segment from $(1,1,0)$ to $\left(\frac{3}{2}, \frac{1}{2}, \frac{1}{2}\right):\left\{(1+t, 1-t, t)\right.$, for $\left.0 \leq t \leq \frac{1}{2}\right\}$.

- The segment from $\left(\frac{3}{2}, \frac{1}{2}, 0\right)$ to $\left(\frac{3}{2}, \frac{1}{2}, \frac{1}{2}\right):\left\{\left(\frac{3}{2}, \frac{1}{2}, t\right)\right.$, for $\left.0 \leq t \leq \frac{1}{2}\right\}$.

- The isolated point: $\left(\frac{5}{2}, \frac{3}{2}, \frac{1}{2}\right)$.

For the case $p=2$ and $q=1$, the product of the two spherical complementary series

$$
C S\left(S O(3,2)_{0}, \delta_{0}\right) \times C S\left(S O(2,1)_{0}, \delta_{0}\right)
$$

is shown in Figure 4. We need to prove that every point in this picture is unitary for $M p(6)$. The unitarity of the unit cube follows from Corollary 4.6. It remains to prove the unitarity of the line segments. Note that every point in a line segment is conjugate to a parameter of the form

$$
\left(\frac{3}{2}, \frac{1}{2}, t\right), \quad 0 \leq t \leq \frac{1}{2}
$$

under the stabilizer of $\delta_{2,1}$. Because $\left(\frac{3}{2}, \frac{1}{2}\right)$ is equal to $\rho_{2}$ (see equation (8.7)), the claim follows immediately from Theorem 8.8. Another proof will be given in the next section. 


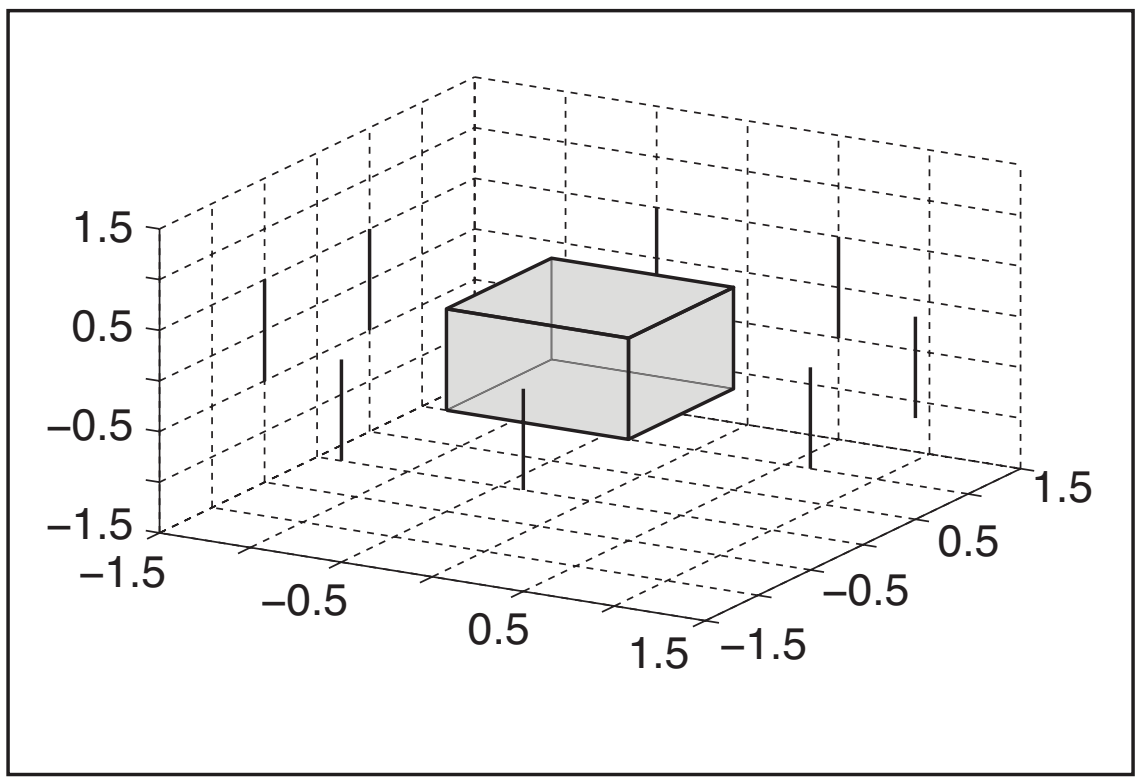

Figure 4. $C S\left(S O(3,2)_{0}, \delta_{0}\right) \times C S\left(S O(2,1)_{0}, \delta_{0}\right)$

8.5. Another family of non-pseudospherical examples. Let $P=M A N$ be a minimal parabolic subgroup of $M p(2 n)$, and let $P_{1}=M_{1} A_{1} N_{1}$ be a parabolic subgroup containing $P$. Then

$$
P \cap M_{1}=M A_{M} N_{M}
$$

is a minimal parabolic subgroup of $M_{1}$, and $A=A_{M} A_{1}$. For each pair of (real) characters $\nu_{M} \in \mathfrak{a}_{M, \mathbb{R}}^{*}$ and $\nu_{1} \in \mathfrak{a}_{1, \mathbb{R}}^{*}$, write $\nu=\left(\nu_{M} \mid \nu_{1}\right)$ for the corresponding character of $A$. Let $\delta$ be a genuine character of $M$, and let $J_{M_{1}}\left(\delta, \nu_{M}\right)$ be the Langlands subquotient of the principal series

$$
\operatorname{Ind}_{M A_{M} N_{M}}^{M_{1}}\left(\delta \otimes \nu_{M} \otimes 1\right) .
$$

Note that $J_{M_{1}}\left(\delta, \nu_{M}\right)$ is always irreducible.

Proposition 8.9. Consider the induced representation

$$
I\left(\nu_{1}\right):=\operatorname{Ind}_{M_{1} A_{1} N_{1}}^{M p(2 n)}\left(J_{M_{1}}\left(\delta, \nu_{M}\right) \otimes \nu_{1} \otimes 1\right)
$$

of $M p(2 n)$. Set $\nu=\left(\nu_{M} \mid \nu_{1}\right)$, and assume that $\nu_{1}$ satisfies:

$$
\begin{cases}\langle\nu, \beta\rangle \notin 2 \mathbb{N}+1 & \text { for all } \beta \in \Delta\left(\mathfrak{n}_{1}\right) \text { that are good for } \delta, \\ \langle\nu, \beta\rangle \notin 2 \mathbb{N}+2 & \text { for all } \beta \in \Delta\left(\mathfrak{n}_{1}\right) \text { that are bad for } \delta .\end{cases}
$$

Then I $\left(\nu_{1}\right)$ is irreducible.

Proof. This follows from Corollary 6.5, (the proof of) Theorem 8 in [12] and Corollary 3.9 in 20.

Corollary 8.10. In the setting of Proposition 8.9 , let $J\left(\nu_{1}\right)$ be the (irreducible) Langlands subquotient of $I\left(\nu_{1}\right)$. Let $R \subset \mathfrak{a}_{1, \mathbb{R}}^{*}$ be any connected region in the complement of the hyperplane arrangement defined in (8.16). If $J\left(\nu_{1}\right)$ is unitary for some value of $\nu_{1}$ in $R$, then $J\left(\nu_{1}\right)$ is unitary throughout the closure of $R$. 
Note that, in our standard notation, $J\left(\nu_{1}\right)=J(\delta, \nu)$ for $\nu=\left(\nu_{M} \mid \nu_{1}\right)$.

Theorem 8.11. Let $G=M p(2 n)$. Choose a genuine representation $\delta=\delta_{p, q}$ of $M$, and let $\nu=\left(\nu^{p} \mid \nu^{q}\right)$, with $\nu^{p}=\left(a_{1}, \ldots, a_{p}\right)$ and $\nu^{q}=\left(a_{p+1}, \ldots, a_{n}\right)$, be a character of $A$ such that

$$
\nu^{p} \in C S\left(S O(p+1, p)_{0}, \delta_{0}\right) \quad \text { and } \quad \nu^{q} \in C S\left(S O(q+1, q)_{0}, \delta_{0}\right) .
$$

Suppose that

- For all $j=1, \ldots, p$, either $0 \leq\left|a_{j}\right| \leq 3 / 2$ or $a_{j} \in \mathbb{Z}+\frac{1}{2}$.

- For all $j=p+1, \ldots, n, 0 \leq\left|a_{j}\right| \leq \frac{1}{2}$ (i.e., $\nu^{q}$ belongs to the unit cube).

Then the Langlands subquotient $J(\delta, \nu)$ of $M p(2 n)$ is unitary.

Proof. Let $P=M A N$ be a minimal parabolic subgroup of $G$, and let $P_{1}=M_{1} A_{1} N_{1}$ be a parabolic subgroup containing $P$, with Levi factor

$$
M_{1} A_{1}:=M p(2 p) \times(\widetilde{G L}(1, \mathbb{R}))^{q} .
$$

The Langlands subquotient of $\operatorname{Ind}_{M_{1} \cap P}^{M_{1}}\left(\delta \otimes \nu^{p} \otimes 1\right)$ is isomorphic to $J\left(\delta_{p, 0}, \nu^{p}\right) \otimes \delta_{0, q}$. Note that $J\left(\delta_{p, 0}, \nu^{p}\right)$ is a pseudospherical irreducible representation of $M p(2 p)$. Given the assumptions on $\nu^{p}$ and Theorem 8.7. $J\left(\delta_{p, 0}, \nu^{p}\right)$ is unitary.

Consider the induced representation

$$
I\left(\nu^{q}\right):=\operatorname{Ind}_{M_{1} A_{1} N_{1}}^{M p(2 n)}\left(\left(J\left(\delta_{p, 0}, \nu^{p}\right) \otimes \delta_{0, q}\right) \otimes \nu^{q} \otimes 1\right),
$$

and let $J\left(\nu^{q}\right)$ be its Langlands subquotient. (Here $J\left(\nu^{q}\right)$ is isomorphic to $J(\delta, \nu)$, for $\nu=\left(\nu^{p} \mid \nu^{q}\right)$.) Up to sign, the roots of $N_{1}$ are:

$$
\begin{array}{ll}
2 \epsilon_{i} & \text { for } p+1 \leq i \leq n, \\
\epsilon_{i} \pm \epsilon_{j} & \text { for } p+1 \leq i<j \leq n, \\
\epsilon_{i} \pm \epsilon_{j} & \text { for } 1 \leq i \leq p \text { and } p+1 \leq j \leq n
\end{array}
$$

The roots in the first two rows are good for $\delta$; the remaining ones are bad. Under our assumptions on $\nu^{p}$, if $\nu^{q}$ is in the interior of the unit cube, then the conditions (8.16) are satisfied. Hence, by Proposition 8.9, $I\left(\nu^{q}\right)$ is irreducible. Moreover, $J(0)=I(0)$ is unitarily induced, hence unitary. By Corollary 8.10, $J\left(\nu^{q}\right)=J(\delta, \nu)$ is unitary for all $\nu^{q}$ in the (closed) unit cube.

We list some applications. First, Theorem 8.11 provides a second proof of the fact that the Langlands quotient

$$
J\left(\delta_{2,1},\left(\frac{3}{2}, \frac{1}{2}, t\right)\right)
$$

of $M p(6)$ is unitary, for all $0 \leq t \leq \frac{1}{2}$.

Second, consider a Langlands subquotient $J\left(\delta_{3,1}, \nu\right)$ of $M p(8)$ with parameter $\nu=\left(\nu^{3} \mid \nu^{1}\right)$ satisfying

$$
\nu^{3} \in C S\left(S O(4,3)_{0}, \delta_{0}\right) \quad \text { and } \quad \nu^{1} \in C S\left(S O(2,1)_{0}, \delta_{0}\right) .
$$

(For an explicit description of $C S\left(S O(4,3)_{0}, \delta_{0}\right)$, see Section 8.4.3.) By inspection, the parameters $\nu^{3}$ and $\nu^{1}$ satisfy all the conditions on $\nu^{p}$ and $\nu^{q}$ in Theorem 8.11 Hence $J\left(\delta_{3,1}, \nu\right)$ is unitary, and Conjecture 8.1 holds for the $\delta_{3,1}$-complementary series of $M p(8)$.

Third, consider the $\delta_{2,2}$-complementary series of $M p(8)$. Theorem 8.11 implies unitarity for all expected parameters, except (possibly) the isolated representation 
$J\left(\delta_{2,2},\left(\frac{3}{2}, \frac{1}{2}, \frac{3}{2}, \frac{1}{2}\right)\right)$. This proves our conjecture for $M p(8)$, with the possible exception of a single unipotent representation. (It turns out that the representation $J\left(\delta_{2,2},\left(\frac{3}{2}, \frac{1}{2}, \frac{3}{2}, \frac{1}{2}\right)\right)$ is also unitary. This claim will be proved in a future paper.)

Finally, observe that all parameters $\nu^{4} \in C S\left(S O(5,4)_{0}, \delta_{0}\right)$ also satisfy the conditions on $\nu^{p}$ in Theorem 8.11 (see Section 11). Therefore, our conjecture holds for the $\delta_{4,1}$-complementary series of $M p(10)$ as well.

\section{Representations of Weyl groups of type $C$}

The stabilizers of genuine $M$-types are subgroups of $W\left(C_{n}\right)$ of the form $W\left(C_{p}\right) \times$ $W\left(C_{q}\right)$, for some pair $(p, q)$ of non-negative integers such that $p+q=n$. In this section, we describe the irreducible representations of Weyl groups of type $C$.

Let $k$ be a non-negative integer. The Weyl group $W\left(C_{k}\right)$ is the semidirect product of the symmetric group $S_{k}$ by the abelian normal subgroup $(\mathbb{Z} / 2 \mathbb{Z})^{k}$ :

$$
W\left(C_{k}\right) \simeq S_{k} \ltimes(\mathbb{Z} / 2 \mathbb{Z})^{k} .
$$

Using the method of "little groups" of Wigner and Mackey (cf. 19, Chapter 7 or 18, Chapter 8), one can show that all irreducible representations of $W\left(C_{k}\right)$ can be obtained as follows.

For non-negative integers $a$ and $b$ such that $a+b=k$, let $\chi=\operatorname{triv}^{a} \otimes \operatorname{sign}^{b}$ be the character of $(\mathbb{Z} / 2 \mathbb{Z})^{k}$ which is trivial on the first $a(\mathbb{Z} / 2 \mathbb{Z})$ factors, and is the sign character on the last $b$. The stabilizer of $\chi$ in $S_{k}$ is isomorphic to $S_{a} \times S_{b}$. Irreducible representations of $S_{a} \times S_{b}$ are parameterized by pairs of partitions $(\lambda, \tau)$, with $\lambda \vdash a$ and $\tau \vdash b$. We write $\lambda \otimes \tau$ for the corresponding representation of $S_{a} \times S_{b}$. Then

$$
[\lambda \otimes \tau] \cdot\left[(\text { triv })^{a} \otimes(\text { sign })^{b}\right]
$$

is a well-defined representation of $\left(S_{a} \times S_{b}\right) \ltimes(\mathbb{Z} / 2 \mathbb{Z})^{k}$. The induced representation

$$
\lambda \times \tau:=\operatorname{Ind}_{\left(S_{a} \times S_{b}\right) \ltimes(\mathbb{Z} / 2 \mathbb{Z})^{k}}^{S_{k} \ltimes(\mathbb{Z} / \mathbb{Z})^{k}}[\lambda \otimes \tau] \cdot\left[(\text { triv })^{a} \otimes(\text { sign })^{b}\right]
$$

is an irreducible representation of $W\left(C_{k}\right)=S_{k} \ltimes(\mathbb{Z} / 2 \mathbb{Z})^{k}$, and all irreducible representations of $W\left(C_{k}\right)$ are obtained this way.

Recall from Theorem 2.8 the relevant representations of $W\left(C_{k}\right)$; they are those of the form $(c, d) \times(0)$ or $(a) \times(b)$.

The representation $(c, d) \times(0)$, which we will denote simply by $(c, d)$ for brevity of notation, is the pull-back to $W\left(C_{k}\right)$ of the irreducible representation of $S_{k}$ corresponding to the partition $(c, d)$ of $k$. It is an irreducible representation of $W\left(C_{k}\right)$ of dimension $\left(\begin{array}{l}k \\ c\end{array}\right)(k-2 c+1) /(k-c+1)$ in which $(\mathbb{Z} / 2 \mathbb{Z})^{k}$ acts trivially. The symbol $(a) \times(b)$ denotes the representation of $W\left(C_{k}\right)$ of dimension $\left(\begin{array}{l}k \\ a\end{array}\right)$ that is induced from the unique representation of $\left(S_{a} \times S_{b}\right) \ltimes(\mathbb{Z} / 2 \mathbb{Z})^{k}$ in which $S_{a}, S_{b}$ and $(\mathbb{Z} / 2 \mathbb{Z})^{a}$ act trivially, and $(\mathbb{Z} / 2 \mathbb{Z})^{b}$ acts by $(\text { sign })^{b}$. In particular, $(k-1) \times(1)$ denotes the natural $k$-dimensional reflection representation of $W\left(C_{k}\right)$. 


\section{Calculations}

In this section, we prove Proposition 5.1 of Section 5.1. Refer to Section 5.1 for the notation.

Proposition. The set $\Sigma_{W}\left(G, \delta_{p, q}\right)$ contains every irreducible relevant representation of $W\left(C_{p}\right)$ and every irreducible relevant representation of $W\left(C_{q}\right)$.

The proof is by direct computation. For every irreducible relevant representation $\tau$ of $W\left(C_{p}\right)$ or $W\left(C_{q}\right)$, we display a petite $K$-type $\mu$ such that the representation of $W^{\delta_{p, q}}=W\left(C_{p}\right) \times W\left(C_{q}\right)$ on the space

$$
V_{\mu}\left[\delta_{p, q}\right]:=\operatorname{Hom}_{M}\left(\mu, \delta_{p, q}\right)
$$

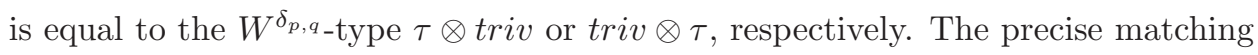
is given in Table 3

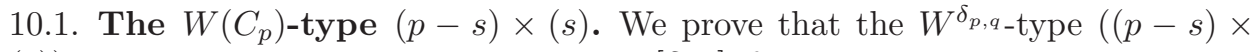
$(s)) \otimes$ triv can be realized on the space $V_{\mu}\left[\delta_{p, q}\right]$, for

$$
\mu=(\underbrace{\frac{1}{2}, \ldots, \frac{1}{2}}_{p-s}, \underbrace{-\frac{1}{2}, \ldots,-\frac{1}{2}}_{q}, \underbrace{-\frac{3}{2}, \ldots,-\frac{3}{2}}_{s}) .
$$

It is convenient to realize the $K$-type $\mu$ inside the tensor product

$$
\rho:=\Lambda^{p-s} \mathbb{C}^{n} \otimes \Lambda^{n-s} \mathbb{C}^{n} \otimes \operatorname{det}^{-3 / 2} .
$$

Note that

$$
\Lambda^{p-s} \mathbb{C}^{n} \otimes \Lambda^{n-s} \mathbb{C}^{n}=\bigoplus_{a=0}^{p-s}(\underbrace{2, \ldots, 2}_{p-s-a}, \underbrace{1, \ldots, 1}_{q+2 a}, \underbrace{0, \ldots, 0}_{s-a}) ;
$$

hence

$$
\rho=\bigoplus_{a=0}^{p-s}(\underbrace{\frac{1}{2}, \ldots, \frac{1}{2}}_{p-s-a}, \underbrace{-\frac{1}{2}, \ldots,-\frac{1}{2}}_{q+2 a}, \underbrace{-\frac{3}{2}, \ldots,-\frac{3}{2}}_{s-a}) .
$$

As an $M$-module,

$$
\rho=\left[\bigoplus_{|T|=p-s} \delta_{T}^{\prime}\right] \otimes\left[\bigoplus_{|S|=n-s} \delta_{S}^{\prime}\right] \otimes \delta_{\emptyset}=\bigoplus_{\substack{|T|=p-s \\|S|=n-s}}^{\bigoplus} \delta_{(T \Delta S)},
$$

where $T \Delta S$ denotes, as usual, the symmetric difference of the two sets. (The notation for $M$-types is as in Section 3.2) Note that $\rho$ contains every $M$-type $\delta_{U}$ with $q \leq|U| \leq q+2(p-s)$ and, in particular, it contains $\delta_{p, q}$. For all $\mathcal{I}=$ $\left\{i_{1}, i_{2}, \ldots, i_{p-s}\right\} \subset\{1,2, \ldots, p\}$, set

$$
v_{\mathcal{I}}:=\left[e_{i_{1}} \wedge e_{i_{2}} \wedge \cdots \wedge e_{i_{p-s}}\right] \otimes\left[e_{i_{1}} \wedge e_{i_{2}} \wedge \cdots \wedge e_{i_{p-s}} \wedge e_{p+1} \wedge e_{p+2} \wedge \cdots \wedge e_{n}\right]
$$

and define

$$
V:=\operatorname{Span}\left\{v_{\mathcal{I}}: \mathcal{I} \subset\{1,2, \ldots, p\},|\mathcal{I}|=p-s\right\} .
$$


It is easy to check that the $\left(\begin{array}{c}p \\ s\end{array}\right)$-dimensional space $V$ is contained in the $\delta_{p, q}$-isotypic component inside $\rho$. We claim that $V$ is actually contained in the $\delta_{p, q}$-isotypic component inside $\mu$ : for all $\mathcal{I}, v_{\mathcal{I}}$ is a weight vector of weight

$$
(-\frac{3}{2}, \ldots,-\frac{3}{2}, \frac{1}{2},-\frac{3}{2}, \ldots,-\frac{3}{2}, \frac{1}{i_{1}} \underset{\substack{\uparrow \\ i_{p-s}}}{\frac{1}{2}},-\frac{3}{2}, \ldots,-\frac{3}{2}, \underbrace{-\frac{1}{2}, \ldots,-\frac{1}{2}}_{q}) .
$$

This weight is conjugate to the highest weight of $\mu$ (and $\rho$ )

$$
\varpi=(\underbrace{\frac{1}{2}, \ldots, \frac{1}{2}}_{p-s}, \underbrace{-\frac{1}{2}, \ldots,-\frac{1}{2}}_{q}, \underbrace{-\frac{3}{2}, \ldots,-\frac{3}{2}}_{s})
$$

by the compact Weyl group; hence $v_{\mathcal{I}}$ must belong to $\mu$.

We study the representation $\psi$ of the stabilizer of $\delta_{p, q}$ on the subspace

$$
\operatorname{Hom}_{M}\left(V, V^{\delta_{p, q}}\right)
$$

of $V_{\mu}\left[\delta_{p, q}\right]$, and prove that it equals $((p-s) \times(s)) \otimes \operatorname{triv}$. (Here $V^{\delta_{p, q}}$ denotes the representation space for $\delta_{p, q}$ )

Recall that $W^{\delta_{p, q}} \simeq W\left(C_{p}\right) \times W\left(C_{q}\right)$. Let $\sigma_{\epsilon_{k}-\epsilon_{l}}$ and $\sigma_{2 \epsilon_{k}}$ be representatives in $K$ for the root reflections $s_{\epsilon_{k}-\epsilon_{l}}$ and $s_{2 \epsilon_{k}}$, as in Section 3.1. Identify $\delta_{p, q}$ with its isotypic component inside the fine $K$-type

$$
\mu_{\delta_{p, q}}=(\underbrace{\frac{1}{2}, \ldots, \frac{1}{2}}_{p}, \underbrace{-\frac{1}{2}, \ldots,-\frac{1}{2}}_{q})=\Lambda^{p} \mathbb{C}^{n} \otimes \operatorname{det}^{-1 / 2},
$$

and write $V^{\delta_{p, q}}=\mathbb{C}\langle w\rangle$, with $w=e_{1} \wedge e_{2} \wedge \cdots \wedge e_{p}$. The elements $v_{\mathcal{I}}$ with $\mathcal{I}=\left\{i_{1}<i_{2} \cdots<i_{p-s}\right\} \subset\{1,2, \ldots, p\}$ form a basis of $V$; let

$$
\left\{T_{\mathcal{J}}: v_{\mathcal{I}} \mapsto \delta_{\mathcal{I}, \mathcal{J}} w\right\}
$$

be the corresponding basis of $\operatorname{Hom}_{M}\left(V, V^{\delta_{p, q}}\right)$. The symbol $\delta_{\mathcal{I}, \mathcal{J}}$ in (10.11) is the Kronecker delta.

If $\alpha=\epsilon_{k}-\epsilon_{l}$, with $1 \leq k<l \leq p$ or $p+1 \leq k<l \leq n$, the element $\sigma_{\alpha}$ acts trivially on both $V$ and $V^{\delta_{p, q}}$; therefore the root reflection $s_{\alpha}$ acts trivially on $\operatorname{Hom}_{M}\left(V, V^{\delta_{p, q}}\right)$. If $\alpha=2 \epsilon_{k}$, then

$$
\mu\left(\sigma_{\alpha}\right) v_{\mathcal{I}}= \begin{cases}-e^{-\frac{3 \pi i}{4}} v_{\mathcal{I}} & \text { if } k \in \mathcal{I} \\ +e^{-\frac{3 \pi i}{4}} v_{\mathcal{I}} & \text { if } k \in\{1,2, \ldots, p\}-\mathcal{I}, \\ i e^{-\frac{3 \pi i}{4}} v_{\mathcal{I}} & \text { if } k \in\{p+1, p+2, \ldots, n\}\end{cases}
$$

and

$$
\mu_{\delta}\left(\sigma_{\alpha}\right) w= \begin{cases}i e^{-\frac{\pi i}{4}} w & \text { if } k \in\{1,2, \ldots, p\} \\ e^{-\frac{\pi i}{4}} w & \text { if } k \in\{p+1, p+2, \ldots, n\}\end{cases}
$$

Hence

$$
\psi\left(s_{\alpha}\right) T_{\mathcal{J}}= \begin{cases}-T_{\mathcal{J}} & \text { if } k \in\{1,2, \ldots, p\}-\mathcal{J} \\ +T_{\mathcal{J}} & \text { if } k \in \mathcal{J} \cup\{p+1, p+2, \ldots, n\}\end{cases}
$$


Let $S_{p-s}$ and $(\mathbb{Z} / 2 \mathbb{Z})^{p-s}$ be the (appropriate) subgroups of $W\left(C_{p}\right)$ acting on the first $p-s$ coordinates, and let $S_{s},(\mathbb{Z} / 2 \mathbb{Z})^{s}$ be the ones acting on the last $s$ coordinates. Note that:

- The restriction of $\psi$ to $W\left(C_{q}\right)$ is trivial; hence $\psi$ is of the form $\psi^{\prime} \otimes$ triv for some representation $\psi^{\prime}$ of $W\left(C_{p}\right)$.

- The groups $S_{p-s}, S_{s}$ and $(\mathbb{Z} / 2 \mathbb{Z})^{p-s}$ act trivially on the vector $T_{\{1, \ldots, p-s\}}$, and $(\mathbb{Z} / 2 \mathbb{Z})^{s}$ acts on it by sign. Hence the restriction of $\psi^{\prime}$ to the group $\left(S_{p-s} \times S_{s}\right) \ltimes(\mathbb{Z} / 2 \mathbb{Z})^{p}$ contains the one-dimensional representation

$$
[(p-s) \otimes(s)] \cdot\left[(\text { triv })^{p-s} \otimes(\text { sign })^{s}\right] .
$$

By Frobenius reciprocity,

$$
\psi^{\prime} \supset(p-s) \times(s)
$$

Actually, $\psi^{\prime}=(p-s) \times(s)$, for dimensional reasons.

We conclude that $\psi=((p-s) \times(s)) \otimes$ triv, as claimed.

10.2. The $W\left(C_{p}\right)$-type $(p-s, s)$. For all $s \in\left\{0,1, \ldots,\left[\frac{p}{2}\right]\right\}$, let $\rho_{s}$ be the tensor product

$$
\rho_{s}:=\Lambda^{s} \mathbb{C}^{n} \otimes \Lambda^{q+s}\left(\mathbb{C}^{n}\right)^{*} \otimes \operatorname{det}^{1 / 2}
$$

Note that

$$
\Lambda^{s} \mathbb{C}^{n} \otimes \Lambda^{q+s}\left(\mathbb{C}^{n}\right)^{*}=\bigoplus_{a=0}^{s}(\underbrace{1, \ldots, 1}_{a}, \underbrace{0, \ldots, 0}_{p-2 a}, \underbrace{-1, \ldots,-1}_{q+a}) ;
$$

hence

$$
\rho_{s}=\bigoplus_{a=0}^{s}(\underbrace{\frac{3}{2}, \ldots, \frac{3}{2}}_{a}, \underbrace{\frac{1}{2}, \ldots, \frac{1}{2}}_{p-2 a}, \underbrace{-\frac{1}{2}, \ldots,-\frac{1}{2}}_{q+a}) .
$$

As an $M$-module,

$$
\rho_{s}=\left[\bigoplus_{|T|=s} \delta_{T}^{\prime}\right] \otimes\left[\bigoplus_{|S|=q+s} \delta_{S}^{\prime}\right] \otimes \delta_{\emptyset}=\underset{\substack{|T|=s \\|S|=q+s}}{\bigoplus} \delta_{T \Delta S}
$$

Then $\rho_{s}$ contains every $M$-type $\delta_{U}$ with $q \leq|U| \leq q+2 s$ and, in particular, it contains the $M$-type $\delta_{p, q}$. The $\delta_{p, q}$-isotypic component inside $\rho_{s}$ is spanned by the vectors:

(10.21) $v_{\mathcal{I}}:=\left[e_{i_{1}} \wedge e_{i_{2}} \wedge \cdots \wedge e_{i_{s}}\right] \otimes\left[f_{i_{1}} \wedge f_{i_{2}} \wedge \cdots \wedge f_{i_{s}} \wedge f_{p+1} \wedge f_{p+2} \wedge \cdots \wedge f_{n}\right]$ with $\mathcal{I}=\left\{i_{1}, i_{2}, \ldots, i_{s}\right\} \subset\{1,2, \ldots, p\}$ (of cardinality $s$ ), and can be identified with the weight space in $\rho_{s}$ corresponding to the weight

$$
\varpi=(\underbrace{\frac{1}{2}, \ldots, \frac{1}{2}}_{p}, \underbrace{-\frac{1}{2}, \ldots,-\frac{1}{2}}_{q}) .
$$


Note that the weight $\varpi$ occurs in each $\rho_{s}$ with multiplicity $\left(\begin{array}{l}p \\ s\end{array}\right)$, and in $\rho_{0}=$ $\Lambda^{q}\left(\mathbb{C}^{n}\right)^{*} \otimes \operatorname{det}^{1 / 2}$ with multiplicity 1 . Because

$$
\rho_{s}=\rho_{s-1} \oplus(\underbrace{\frac{3}{2}, \ldots, \frac{3}{2}}_{s}, \underbrace{\frac{1}{2}, \ldots, \frac{1}{2}}_{p-2 s}, \underbrace{-\frac{1}{2}, \ldots,-\frac{1}{2}}_{q+s}), \quad \forall s \in\left\{1, \ldots,\left[\frac{p}{2}\right]\right\},
$$

we conclude that the $K$-type

$$
\mu_{s}:=(\underbrace{\frac{3}{2}, \ldots, \frac{3}{2}}_{s}, \underbrace{\frac{1}{2}, \ldots, \frac{1}{2}}_{p-2 s}, \underbrace{-\frac{1}{2}, \ldots,-\frac{1}{2}}_{q+s})
$$

contains $\delta_{p, q}$ with multiplicity

$$
m_{s}=\left(\begin{array}{l}
p \\
s
\end{array}\right)-\left(\begin{array}{c}
p \\
s-1
\end{array}\right)
$$

for all $s \in\left\{1, \ldots,\left[\frac{p}{2}\right]\right\}$. Note that the multiplicity of $\delta_{p, q}$ in $\mu_{0}$ is 1 , so the previous formula holds also for $s=0$ if we let $\left(\begin{array}{c}p \\ -1\end{array}\right)=0$.

The purpose of this section is to compute the representation $\psi_{s}$ of the group $W^{\delta_{p, q}} \simeq W\left(C_{p}\right) \times W\left(C_{q}\right)$ on the space $V_{\mu_{s}}\left[\delta_{p, q}\right]$ and to prove that it equals $(p-s, s) \otimes$ triv.

It is convenient to first look at the representation of $W^{\delta_{p, q}}$ on the larger space $V_{\rho_{s}}\left[\delta_{p, q}\right]$, which we denote by $\tilde{\psi}_{s}$. We identify $\delta_{p, q}$ with its isotypic component inside the fine $K$-type $\mu_{\delta_{p, q}}=\Lambda^{p} \mathbb{C}^{n} \otimes \operatorname{det}^{-1 / 2}$ and choose the bases

$$
w=e_{1} \wedge e_{2} \wedge \cdots \wedge e_{p}
$$

of $V^{\delta_{p, q}}$ and

$$
\left\{v_{\mathcal{I}}: \mathcal{I} \subset\{1,2, \ldots, p\},|\mathcal{I}|=s\right\}
$$

of the $\delta_{p, q}$-isotypic component in $\rho_{s}$. The maps

$$
T_{\mathcal{J}}: v_{\mathcal{I}} \rightarrow \delta_{\mathcal{I}, \mathcal{J}} w
$$

form a basis of $V_{\rho_{s}}\left[\delta_{p, q}\right]$. If $\alpha=2 \epsilon_{k}$, then

$$
\mu\left(\sigma_{\alpha}\right) v_{\mathcal{I}}= \begin{cases}e^{\frac{\pi i}{4}} v_{\mathcal{I}} & \text { if } k \in\{1,2, \ldots, p\}, \\ e^{-\frac{\pi i}{4}} v_{\mathcal{I}} & \text { if } k \in\{p+1, p+2, \ldots, n\}\end{cases}
$$

and

$$
\mu_{\delta}\left(\sigma_{\alpha}\right) w= \begin{cases}e^{\frac{\pi i}{4}} w & \text { if } k \in\{1,2, \ldots, p\} \\ e^{-\frac{\pi i}{4}} w & \text { if } k \in\{p+1, p+2, \ldots, n\}\end{cases}
$$

Hence

$$
\tilde{\psi}_{s}\left(s_{\alpha}\right) T_{\mathcal{J}}=T_{\mathcal{J}}, \quad \forall \mathcal{J} .
$$

This proves that the representation $\tilde{\psi}_{s}$ of $W\left(C_{p}\right) \times W\left(C_{q}\right)$ on $V_{\rho_{s}}\left[\delta_{p, q}\right]$ is actually a representation of $S_{p} \times S_{q}$. Now set $\alpha=\epsilon_{k}-\epsilon_{l}$. If $p+1 \leq k<l \leq n$, then

$$
\mu\left(\sigma_{\alpha}\right) v_{\mathcal{I}}=v_{\mathcal{I}}, \quad \forall \mathcal{I}
$$


and

$$
\mu_{\delta}\left(\sigma_{\alpha}\right) w=w .
$$

Again, we find that $\tilde{\psi}_{s}\left(s_{\alpha}\right) T_{\mathcal{J}}=T_{\mathcal{J}}, \forall \mathcal{J}$. So the symmetric group $S_{q}$ acts trivially, and we are really dealing with a representation of $S_{p}$. If $\alpha=\epsilon_{k}-\epsilon_{l}$ with $1 \leq k<$ $l \leq p$, then

$$
\mu\left(\sigma_{\alpha}\right) v_{\mathcal{I}}= \begin{cases}v_{\mathcal{I}} & \text { if }\{k, l\} \subseteq \mathcal{I} \text { or }\{k, l\} \subseteq\{1, \ldots, p\}-\mathcal{I}, \\ v_{\mathcal{I} \triangle\{k, l\}} & \text { otherwise }\end{cases}
$$

and $\mu_{\delta_{p, q}}\left(\sigma_{\alpha}\right) w=w$. It is easy to check that the symmetric group $S_{p}$ permutes the subspaces

$$
U_{\mathcal{I}}:=\mathbb{C} v_{\mathcal{I}} \quad \mathcal{I} \subseteq\{1, \ldots, p\},|\mathcal{I}|=s
$$

transitively, and that the space $V_{\rho_{s}}\left[\delta_{p, q}\right]$ (on which the representation $\tilde{\psi}_{s}$ is defined) decomposes as the direct sum of the $U_{\mathcal{I}}$ 's:

$$
V_{\rho_{s}}\left[\delta_{p, q}\right]=\bigoplus_{\mathcal{I} \subseteq\{1, \ldots, p\},|\mathcal{I}|=s} U_{\mathcal{I}} .
$$

Set $\mathcal{I}_{0}=\{1,2, \ldots, s\}$ and $U=U_{\mathcal{I}_{0}}$, and let $H$ be the stabilizer of $U$ in $S_{p}$ (i.e., the set of all $\eta$ in $S_{p}$ such that $\left.\eta U=U\right)$. Then

- $U$ is stable under $H$, and

- the $S_{p}$-module $V_{\rho_{s}}\left[\delta_{p, q}\right]$ is induced from the $H$-module $U$

(cf. 18, Proposition 19). Because $U$ is isomorphic to the trivial representation of $H$, and $H$ is isomorphic to $S_{p-s} \times S_{s}$, we find that:

$$
\left.\tilde{\psi}_{s}\right|_{S_{p}}=\operatorname{Ind}_{S_{p-s} \times S_{s}}^{S_{p}} \text { triv }=M^{(p-s, s)} .
$$

The permutation module $M^{(p-s, s)}$ of $S_{p}$ decomposes as a direct sum of Specht modules:

$$
M^{(p-s, s)}=\bigoplus_{\lambda \unrhd(p-s, s)} \mathcal{S}^{\lambda}=\bigoplus_{a=0}^{s} \mathcal{S}^{(p-a, a)}
$$

(with abuse of notation we let $(p-0,0)$ denote the trivial partition). Then

$$
\left.\tilde{\psi}_{s}\right|_{W\left(C_{p}\right)}=\bigoplus_{a=0}^{s}(p-a, a)
$$

and

$$
\tilde{\psi}_{s}=\bigoplus_{a=0}^{s}(p-a, a) \otimes \text { triv }
$$

We can also write

$$
\tilde{\psi}_{s}=V_{\rho_{s}}\left[\delta_{p, q}\right]=V_{\bigoplus_{a=0}^{s} \mu_{a}}\left[\delta_{p, q}\right]=\bigoplus_{a=0}^{s} V_{\mu_{a}}\left[\delta_{p, q}\right]=\bigoplus_{a=0}^{s} \psi_{a} .
$$

In order to prove that $\psi_{a}=(p-a, a) \otimes$ triv for all $a$, it is sufficient to show that the result holds for $a=0$ :

$$
\psi_{0}=(p) \otimes t r i v=t r i v \otimes t r i v ;
$$


the rest of the claim will follow by induction. This is easy to do: the $K$-type $\mu_{0}$ is the unique fine $K$-type $\mu_{\delta_{p, q}}$ containing $\delta_{p, q}$; hence the representation $\psi_{0}=V_{\mu_{0}}\left[\delta_{p, q}\right]$ is trivial. We conclude that

$$
\psi_{a}=(p-a, a) \otimes t r i v
$$

for all $a=0, \ldots, s$. In particular, $\psi_{s}=(p-s, s) \otimes$ triv.

10.3. Relevant $W\left(C_{q}\right)$-types. Similar arguments prove that the $W^{\delta_{p, q} \text {-types }}$

$$
\text { triv } \otimes((q-r) \times(r)) \quad \text { and } \quad \text { triv } \otimes(q-r, r)
$$

can be realized on the space $V_{\mu}\left[\delta_{p, q}\right]$, for

$$
\mu=(\underbrace{\frac{3}{2}, \ldots, \frac{3}{2}}_{r}, \underbrace{\frac{1}{2}, \ldots, \frac{1}{2}}_{p}, \underbrace{-\frac{1}{2}, \ldots,-\frac{1}{2}}_{q-r})
$$

and

$$
\mu=(\underbrace{\frac{1}{2}, \ldots, \frac{1}{2}}_{p+r}, \underbrace{-\frac{1}{2}, \ldots,-\frac{1}{2}}_{q-2 r}, \underbrace{-\frac{3}{2}, \ldots,-\frac{3}{2}}_{r}),
$$

respectively. The details are left to the reader.

11. Appendix: The spherical unitary dual of $S O(n+1, n)_{0}$.

In this section, we give an explicit description of the spherical unitary dual of split groups of type $B$. All the results are known, and due to D. Barbasch (see [4] or [6); we include them here for convenience.

Let $G=S O(n+1, n)_{0}$ be the real split connected group of type $B_{n}$ and let $\check{\mathfrak{g}}=\mathfrak{s p}(2 n, \mathbb{C})$ be the complex dual Lie algebra. The spherical unitary dual of $G$ is a disjoint union of sets, called "complementary series", each parameterized by a nilpotent orbit in $\mathfrak{\mathfrak { g }}$. Recall that nilpotent orbits in $\mathfrak{s p}(2 n, \mathbb{C})$ are parameterized by partitions of $2 n$ in which every odd part occurs with even multiplicity.

Definition 11.1. Let $\chi$ be a real parameter in $\check{\mathfrak{h}}$, and let $\check{\mathcal{O}}$ be a nilpotent orbit in $\check{\mathfrak{g}}$. We say that " $\chi$ is attached to $\check{\mathcal{O}}$ " if

(1) $\chi=\check{h} / 2+\nu$, for some $\nu \in \mathfrak{Z}_{\mathfrak{g}}(\check{\mathcal{O}})$ semisimple, and

(2) whenever $\check{\mathcal{O}}^{\prime}$ is another nilpotent orbit in $\check{\mathfrak{g}}$ such that $\chi=\check{h}^{\prime} / 2+\nu^{\prime}$, for some $\nu^{\prime} \in \mathfrak{Z}_{\check{\mathfrak{g}}}\left(\check{\mathcal{O}}^{\prime}\right)$ semisimple, then $\check{\mathcal{O}}^{\prime} \subset \check{\mathcal{O}}$.

If $\chi$ is a real parameter in $\check{\mathfrak{h}}$, we can identify $\chi$ with an element of $\mathfrak{a}_{\mathbb{R}}^{*}$ and consider the irreducible spherical representation $J\left(\delta_{0}, \chi\right)$ of $G$.

Definition 11.2. A representation $J\left(\delta_{0}, \chi\right)$ is in the "complementary series for $\breve{\mathcal{O}}$ " if

(1) $\chi$ is a parameter attached to $\check{\mathcal{O}}$, and

(2) $J\left(\delta_{0}, \chi\right)$ is unitary; i.e., $\chi \in C S\left(G, \delta_{0}\right)$.

The zero-complementary series, i.e., the complementary series attached to the trivial nilpotent orbit, plays a special role. 


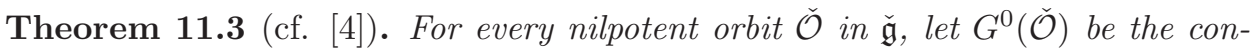
nected real split group whose complex dual Lie algebra is $\mathfrak{\mathfrak { Z }}_{\mathfrak{\mathfrak { g }}}(\check{\mathcal{O}})$. Let $\chi=\frac{1}{2} \check{h}+\nu$ be a parameter attached to the nilpotent orbit $\check{\mathcal{O}}$. Then $\chi$ is in the $\check{\mathcal{O}}$-complementary series for the group $G$ if and only if $\nu$ is in the zero-complementary series for the group $G^{0}(\check{\mathcal{O}})$.

(Note that $\nu \in \mathfrak{Z}_{\mathfrak{\mathfrak { g }}}(\check{\mathcal{O}})$, so we can think of $\nu$ as a spherical parameter for $G^{0}(\check{\mathcal{O}})$.) The zero-complementary series of all real split groups is known, thanks to the work of D. Barbasch. If $G=S O(n+1, n)_{0}$, the group $G^{0}(\check{\mathcal{O}})$ can only involve factors of type $B_{k}, C_{k}$ and $D_{k}$. We recall the zero-complementary series for these groups.

Theorem 11.4 (cf. 4]). The zero-complementary series for split groups of type $B_{k}, C_{k}$ and $D_{k}$ consists of the following dominant parameters:

$B_{k}$. The set of all $\chi=\left(\nu_{1}, \ldots, \nu_{k}\right)$ such that $0 \leq \nu_{1} \leq \nu_{2} \leq \cdots \leq \nu_{k}<\frac{1}{2}$.

$C_{k}$. The set of all $\chi=\left(\nu_{1}, \ldots, \nu_{k}\right)$ such that there exists an index $i=2, \ldots, k$ with the property that

$$
0 \leq \nu_{1} \leq \cdots \leq \nu_{i}<1-\nu_{i-1}<\nu_{i+1}<\cdots<\nu_{k}<1
$$

and, for every $i \leq j<k$, there is an odd number of $\nu_{l}$ with $1 \leq l<i$ such that $\nu_{j}<1-\nu_{l}<\nu_{j+1}$.

$D_{k}$. Similar to type $C_{k}$. If $k$ is even, replace every occurrence of $\nu_{1}$ by $\left|\nu_{1}\right|$. If $k$ is odd, replace every occurrence of $\nu_{1}$ by 0 .

Note that the choice of simple roots (and dominant parameters) is not the standard one. As Theorem 11.4 shows, the zero-complementary series for a split group of type $B$ reduces to the fundamental alcove, whereas the ones for types $C$ and $D$ are a bit more complicated. Here are some low rank examples.

$C_{1} .0 \leq \nu_{1}<1$.

$D_{1} . \nu_{1}=0$.

$C_{2} .0 \leq \nu_{1} \leq \nu_{2}<1-\nu_{1}<1$.

$D_{2} .0 \leq\left|\nu_{1}\right| \leq \nu_{2}<1-\left|\nu_{1}\right|<1$.

$C_{3} .0 \leq \nu_{1} \leq \nu_{2}<1-\nu_{1}<\nu_{3}<1$ or $0 \leq \nu_{1} \leq \nu_{2} \leq \nu_{3}<1-\nu_{2}<1$.

$D_{3} . \nu_{1}=0$ and $0 \leq \nu_{2} \leq \nu_{3}<1-\nu_{2}<1$.

$C_{4} .0 \leq \nu_{1} \leq \nu_{2} \leq \nu_{3}<1-\nu_{2}<\nu_{4}<1-\nu_{1}<1$ or $0 \leq \nu_{1} \leq \nu_{2} \leq \nu_{3} \leq \nu_{4}<$

$1-\nu_{3}<1$.

$D_{4} .0 \leq\left|\nu_{1}\right| \leq \nu_{2} \leq \nu_{3}<1-\nu_{2}<\nu_{4}<1-\left|\nu_{1}\right|<1$ or $0 \leq\left|\nu_{1}\right| \leq \nu_{2} \leq \nu_{3} \leq$ $\nu_{4}<1-\nu_{3}<1$.

Let us go back to the problem of describing the spherical unitary dual of $S O(n+1, n)_{0}$. In order to determine the complementary series attached to an orbit $\check{\mathcal{O}}$ in $\check{\mathfrak{g}}$, we need to:

(1) Find the group $G^{0}(\check{\mathcal{O}})$.

(2) Find all the parameters $\chi=\frac{1}{2} \check{h}+\nu$ attached to $\check{\mathcal{O}}$.

(3) Impose that $\nu$ belongs to the zero-complementary series of $G^{0}(\check{\mathcal{O}})$.

Let $\lambda$ be the partition corresponding to the nilpotent orbit $\check{\mathcal{O}}$; denote the parts of $\lambda$ by $a_{l}$, and their multiplicity by $r_{l}$ :

$$
\lambda=(\underbrace{a_{1}, \ldots, a_{1}}_{r_{1}}, \ldots, \underbrace{a_{m}, \ldots, a_{m}}_{r_{m}})
$$


$\left(r_{l}\right.$ is even if $a_{l}$ is odd). The centralizer $\mathfrak{Z}_{\check{\mathfrak{g}}}(\check{\mathcal{O}})$ of $\check{\mathcal{O}}$ in $\check{\mathfrak{g}}$ is a direct sum of symplectic and orthogonal Lie algebras. Precisely, there is a summand $\mathfrak{s p}\left(r_{l}\right)$ coming from each odd part, and a summand $\mathfrak{s o}\left(r_{l}\right)$ coming from each even part.

Example 11.5. If $\lambda=\left(6^{4} 2^{3} 1^{8}\right)$, then $\mathfrak{Z}_{\mathfrak{g}}(\check{\mathcal{O}})$ is of type $D_{2} \times B_{1} \times C_{4}$ :

$$
\mathfrak{Z}_{\check{\mathfrak{g}}}(\check{\mathcal{O}})=\mathfrak{s o}(4) \oplus \mathfrak{s o}(3) \oplus \mathfrak{s p}(8) \text {. }
$$

Hence $G^{0}(\check{\mathcal{O}})$ is of type $D_{2} \times C_{1} \times B_{4}$ :

$$
G^{0}(\check{\mathcal{O}})=S O(2,2)_{0} \times S p(2) \times S O(5,4)_{0} .
$$

We describe the contribution of each part of $\lambda$ to $\chi=\frac{1}{2} \check{h}+\nu$, and show how to determine the restrictions on $\nu$ that make $\chi$ unitary.

If $a$ is an odd part of $\lambda$, its multiplicity is necessarily even. If $r_{a}=2 n_{a}$, the partition $\lambda$ contains $n_{a}$ pairs of the form $(a, a)$. The $j^{\text {th }}$ pair $(a, a)$ contributes a string

$$
-\left(\frac{a-1}{2}\right),-\left(\frac{a-3}{2}\right), \ldots,-1,0,+1, \ldots,+\left(\frac{a-3}{2}\right),+\left(\frac{a-1}{2}\right)
$$

(of length $a$ ) to $\frac{1}{2} \check{h}$, and a string

$$
\left(\nu_{j}^{a}, \nu_{j}^{a}, \ldots, \nu_{j}^{a}\right)
$$

(also of length $a$ ) to $\nu$. Moreover, the part $a$ contributes a summand $\mathfrak{s p}\left(2 n_{a}\right)$ to the stabilizer of the orbit, and a factor $S O\left(n_{a}+1, n_{a}\right)_{0}$ to the group $G^{0}(\check{\mathcal{O}})$. To find the possible values of the $\nu_{j}^{a}$ 's, we impose the condition that the parameter

$$
\left(\nu_{1}^{a}, \ldots, \nu_{n_{a}}^{a}\right)
$$

belongs to the zero-complementary series for $S O\left(n_{a}+1, n_{a}\right)_{0}$ (see Theorem 11.4).

If $a$ is an even part of $\lambda$, its multiplicity $r_{a}$ can be even or odd. We discuss the two cases separately. If $r_{a}=2 n_{a}$, the partition $\lambda$ contains $n_{a}$ pairs of the form $(a, a)$. The $j^{\text {th }}$ pair $(a, a)$ contributes a string

$$
-\left(\frac{a-1}{2}\right),-\left(\frac{a-3}{2}\right), \ldots,-\frac{1}{2},+\frac{1}{2}, \ldots,+\left(\frac{a-3}{2}\right),+\left(\frac{a-1}{2}\right)
$$

(of length $a$ ) to $\frac{1}{2} \check{h}$, and a string

$$
\left(\nu_{j}^{a}, \nu_{j}^{a}, \ldots, \nu_{j}^{a}\right)
$$

(also of length $a$ ) to $\nu$. Moreover, the part $a$ contributes a summand $\mathfrak{s o}\left(2 n_{a}\right)$ to the stabilizer of the orbit, and a factor $S O\left(n_{a}, n_{a}\right)_{0}$ to the group $G^{0}(\check{\mathcal{O}})$. To find the possible values of the $\nu_{j}^{a}$ 's, we require that the parameter

$$
\left(\nu_{1}^{a}, \ldots, \nu_{n_{a}}^{a}\right)
$$

belongs to the zero-complementary series for $S O\left(n_{a}, n_{a}\right)_{0}$. If $r_{a}=2 n_{a}+1$, the partition $\lambda$ contains $n_{a}$ pairs of the form $(a, a)$ and a "singleton" $a$. The contributions of each pair $(a, a)$ to $\frac{1}{2} \check{h}$ and to $\nu$ are the same as before. The singleton contributes a string

$$
\left(\frac{1}{2}, \frac{3}{2}, \ldots, \frac{a-1}{2}\right)
$$

(of length $a / 2$ ) to $\frac{1}{2} \check{h}$, and a string of zeros to $\nu$ (also of length $a / 2$ ). The part $a$ also contributes a summand $\mathfrak{s o}\left(2 n_{a}+1\right)$ to the stabilizer of the orbit, and a 
factor $S p\left(2 n_{a}\right)$ to the group $G^{0}(\check{\mathcal{O}})$. To find the range for the $\nu_{j}^{a}$ 's, we impose the condition that the parameter

$$
\left(\nu_{1}^{a}, \ldots, \nu_{n_{a}}^{a}\right)
$$

belongs to the zero-complementary series for $S p\left(2 n_{a}\right)$.

Example 11.6 (continued). To continue with our example, the complementary series of $G=S O(20,19)_{0}$ attached to the orbit given by $\lambda=\left(6^{4} 2^{3} 1^{8}\right)$ is

$$
\begin{aligned}
&\left\{\left(-\frac{5}{2}+\nu_{1},\right.\right.-\frac{3}{2}+\nu_{1},-\frac{1}{2}+\nu_{1}, \frac{1}{2}+\nu_{1}, \frac{3}{2}+\nu_{1}, \frac{5}{2}+\nu_{1}, \\
&-\frac{5}{2}+\nu_{2},-\frac{3}{2}+\nu_{2},-\frac{1}{2}+\nu_{2}, \frac{1}{2}+\nu_{2}, \frac{3}{2}+\nu_{2}, \frac{5}{2}+\nu_{2}, \\
&\left.\left.\frac{1}{2},-\frac{1}{2}+\nu_{3}, \frac{1}{2}+\nu_{3}, \nu_{4}, \nu_{5}, \nu_{6}, \nu_{7}\right)\right\}
\end{aligned}
$$

with

$$
0 \leq\left|\nu_{1}\right| \leq \nu_{2}<1-\left|\nu_{1}\right|<1, \quad 0 \leq \nu_{3}<1 \quad \text { and } \quad 0 \leq \nu_{4} \leq \nu_{5} \leq \nu_{6} \leq \nu_{7}<\frac{1}{2} .
$$

\section{REFERENCES}

1. J. Adams, Nonlinear covers of real groups, Int. Math. Res. Not. 75, 2004, 4031-4047. MR2112326 (2006c:20096)

2. J. Adams, D. Barbasch, Genuine representations of the metaplectic group, Comp. Math. 113, 1998, 23-66. MR:1638210 (99h:22013)

3. J. Adams, D. Barbasch, A. Paul, P. Trapa, D.A. Vogan, Jr., Unitary Shimura correspondences for split real groups, J. Amer. Math. Soc. 20, 2007, 701-751. MR2291917 (2008i:22008)

4. D. Barbasch, Unitary spherical spectrum for split classical groups, preprint, arXiv:math/ 0609828v4 [math.RT].

5. - Relevant and petite $K$-types for split groups, Functional analysis VIII, 35-71, Various Publ. Ser. (Aarhus), 47, Aarhus Univ., Aarhus, 2004. MR2127163(2006d:22020)

6. D. Barbasch, D. Ciubotaru, A. Pantano, Unitarizable minimal principal series of reductive groups, Contemp. Math. 472, Amer. Math. Soc., 2008, 63-136. MR.2454332

7. D. Barbasch, A. Moy, Reduction to real infinitesimal character in affine Hecke algebras, J. Amer. Math. Soc., 6, 1993, 611-635. MR.1186959 (93k:22015)

8. D. Barbasch, A. Pantano, Petite K-types for nonspherical minimal principal series, preprint.

9. R. Howe, Transcending classical invariant theory, J. Amer. Math. Soc. 2, 1989, 535-552. MR $985172(90 \mathrm{k}: 22016)$

10. D. Kazhdan, G. Lusztig, Proof of the Deligne-Langlands conjecture for Hecke algebras, Invent. Math., 87, 1987, 153-215. MR862716 (88d:11121)

11. A.W. Knapp, Representation Theory of Semisimple Groups. An Overview Based on Examples, Princeton University Press, Princeton, NJ, 2001. MR.1880691 (2002k:22011)

12. A.W. Knapp, G.J. Zuckerman, Classification theorems for representations of semisimple Lie groups, Lecture Notes in Mathematics 587, 1977, 138-159. MR0476923 (57:16474)

13. J.-S. Li, Singular unitary representations of classical groups, Invent. Math. 97, 1989, 237-255. MR 1001840 (90h:22021)

14. A. Pantano, A. Paul, S. Salamanca-Riba, The omega-regular unitary dual of the metaplectic group of rank 2, Contemp. Math. 467, Amer. Math. Soc., 2008, 1-47. MR2484675

15. - The genuine omega-regular unitary dual of the metaplectic group, preprint.

16. T. Przebinda, The duality correspondence of infinitesimal characters, Colloq. Math. 70, 1996, 93-102. MR1373285 (96m:22034)

17. S. Salamanca-Riba, On the unitary dual of some classical Lie groups, Comp. Math. 68, 1988, 251-303. MR971329 (89m:22018)

18. J.-P. Serre, Linear Representations of Finite Groups, Graduate Texts in Mathematics, Vol. 42, Springer, 1996. MR 0450380 (56:8675)

19. D.J. Simms, Lie Groups and Quantum Mechanics, Lecture Notes in Mathematics, vol. 52, Springer, 1968. MR 0232579(38:904) 
20. B. Speh, D.A. Vogan, Jr., Reducibility of generalized principal series representations, Acta Math. 145, 1980, 227-299. MR590291 (82c:22018)

21. D.A. Vogan, Jr., Representations of Real Reductive Lie Groups, Progress in Mathematics, Vol. 15, Birkhäuser, 1981. MR632407 (83c:22022)

Department of Mathematics, University of California at Irvine, Irvine, California 92697

E-mail address: apantano@math.uci.edu

Department of Mathematics, Western Michigan University, Kalamazoo, Michigan 49008

E-mail address: annegret.paul@wmich.edu

Department of Mathematics, New Mexico State University, Las Cruces, New Mexico 88003

E-mail address: ssalaman@nmsu.edu 